\title{
Intersection theory of punctured pseudoholomorphic curves
}

\author{
RICHARD SIEFRING
}

\begin{abstract}
We study the intersection theory of punctured pseudoholomorphic curves in 4dimensional symplectic cobordisms. Using the asymptotic results of the author [22], we first study the local intersection properties of such curves at the punctures. We then use this to develop topological controls on the intersection number of two curves. We also prove an adjunction formula which gives a topological condition that will guarantee a curve in a given homotopy class is embedded, extending previous work of Hutchings [14].

We then turn our attention to curves in the symplectization $\mathbb{R} \times M$ of a 3 -manifold $M$ admitting a stable Hamiltonian structure. We investigate controls on intersections of the projections of curves to the 3-manifold and we present conditions that will guarantee the projection of a curve to the 3-manifold is an embedding.

Finally we consider an application concerning pseudoholomorphic curves in manifolds admitting a certain class of holomorphic open book decomposition and an application concerning the existence of generalized pseudoholomorphic curves, as introduced by Hofer [7].
\end{abstract}

32Q65; 53D42, 57R58

\section{Introduction}

In this paper we will study the intersection theory of punctured pseudoholomorphic curves which arise in symplectic field theory (Eliashberg, Givental and Hofer [4]). Positivity of intersections for pseudoholomorphic curves has been an important tool in applications of pseudoholomorphic curves to 4-dimensional symplectic topology. First stated by Gromov in [6], rigorous proofs were subsequently provided by McDuff [17], and Micallef and White [18]. Put simply, positivity of intersections states that isolated intersections between two curves contribute positively to their intersection number and that singular points contribute positively to the self-intersection number of a single curve. These local statements translate into useful global topological statements for closed pseudoholomorphic curves. If $u:(\Sigma, j) \rightarrow(M, J)$ and $v:\left(\Sigma^{\prime}, j^{\prime}\right) \rightarrow(M, J)$ are pseudoholomorphic maps with closed connected domains and nonidentical images, then the intersection number $[u] \cdot[v]$ is always nonnegative and $[u] \cdot[v]=0$ if and only 
if $u$ and $v$ do not intersect. Moreover, if $u:(\Sigma, j) \rightarrow(M, J)$ is a simple, closed, pseudoholomorphic map, then $u$ satisfies the inequality

$$
[u] \cdot[u]-\left\langle c_{1}(T M, J),[u]\right\rangle+\chi(\Sigma) \geq 0
$$

and equality occurs if and only if $u$ is an embedding.

While the local results on the intersections of pseudoholomorphic curves apply to punctured curves appearing in symplectic field theory, finding generalizations of the global results is subtle due to the fact that the intersection number is no longer homotopy invariant when the domains of the curves are noncompact. Indeed, in a pair of smooth homotopies of curves, intersections can escape or appear at the ends. One way to deal with this issue is to perturb one of the curves being considered near the ends and compute the intersection number between one curve and the perturbation of the second curve. Given a sufficiently precise description of the asymptotic behavior, it is then possible to compute this intersection number in terms of the intersection number of the original curves and behavior near the punctures. This idea was studied by Kriener in [16], where the self-intersection number of a single embedded half-cylinder asymptotic to a multiply covered orbit is considered. These ideas were further pursued by Hutchings [14; 15], who proved an index inequality for curves in symplectizations. This index inequality, important for the foundations of embedded contact homology, gives a topological criterion that will guarantee a curve is embedded and has asymptotic behavior which satisfies a technical "admissibility" condition.

The goal of the present paper is to further develop some of the techniques used in [14] and study algebraic controls on intersections and embeddedness of punctured pseudoholomorphic curves. We first give a complete study of the local "asymptotic intersection theory," made possible by the asymptotic descriptions of curves in [22]. With this in hand, we introduce the notion of the "generalized intersection number" of two smooth proper maps from punctured Riemann surfaces which are asymptotic at the punctures to cylinders over periodic orbits. From the results we prove about asymptotic intersection theory, it will follow that the generalized intersection number of two distinct pseudoholomorphic maps is always nonnegative and is equal to zero if and only if the curves do not intersect and the curves do not have any tangencies at infinity, where the notion of tangency at infinity can be made precise in terms of the asymptotic description from [22]. Moreover, we state a generalization of the adjunction formula (1-1) in terms of the generalized intersection number.

Having addressed these things we specialize to the case of a cylindrical cobordism $\mathbb{R} \times M$ equipped with an $\mathbb{R}$-invariant almost complex structure. Here we relate the number of intersections of two curves with the number of intersections of each curve 
with the asymptotic limits of the other and the winding of the curves around their asymptotic limits. This allows us to state a set of necessary and sufficient conditions that will guarantee that the projection of the two curves to the 3-manifold $M$ do not intersect. These same techniques, used with the adjunction formula for punctured curves, allow us to give conditions that will guarantee the projection of a curve to the 3-manifold is embedded. These results and ideas are useful in the study of finite energy foliations, as initiated by Hofer, Wysocki and Zehnder [12]. As an application of these results, we prove a result about the contact homology of a 3-manifold admitting a special class of holomorphic open book decompositions.

Finally, we consider so-called generalized pseudoholomorphic curves as introduced by Hofer in [7]. We show that the generalized intersection product can be used to develop topological obstructions to the existence of these curves.

Acknowledgements This paper has its roots in work I began as a graduate student and I would like to thank my advisor, Helmut Hofer, for his encouragement and support.

During the writing of this paper, I have had many in-depth conversations about this material which have helped to clarify my thinking and have shaped the exposition here. In particular I would like to thank Barney Bramham, Michael Hutchings, Al Momin, Eric Schoenfeld and Chris Wendl for helpful conversations and for their interest in this work.

\section{Background and main results}

\subsection{Hamiltonian structures}

In this section we describe a structure defined on 3-manifolds that is central to all we do here. For further background and examples, see Bourgeois, Eliashberg, Hofer, Wysocki and Zehnder [2, Section 2] or Eliashberg, Kim and Polterovich [5, Example 4.2].

Let $M$ be a compact oriented 3-manifold equipped with a pair $\mathcal{H}=(\lambda, \omega)$ where $\lambda$ is a $1-$ form and $\omega$ is a 2 -form on $M$. Assume that

(H1) $\lambda \wedge \omega$ is a volume form on $M$.

Then $\omega$ must be rank 2 everywhere and thus defines a line bundle $\ell_{\omega} \subset T M$ by

$$
\ell_{\omega}=\cup_{p \in M}\left(p, \operatorname{ker} \omega_{p}\right)
$$


where $\operatorname{ker} \omega_{p}$ is the kernel of the linear map $\omega_{p}: T M_{p} \rightarrow T^{*} M_{p}$ defined by $v \mapsto$ $\omega_{p}(v, \cdot)$. The condition (H1) implies that $\lambda$ is non-zero on $\ell_{\omega}$, that the hyperplane distribution defined by

$$
\xi^{\mathcal{H}}:=\operatorname{ker} \lambda
$$

is everywhere transverse to $\ell_{\omega}$ and that $\omega$ is nondegenerate on $\xi^{\mathcal{H}}$. If we define a vector field $X_{\mathcal{H}}$ to be the unique section of $\ell_{\omega}$ satisfying $\lambda\left(X_{\mathcal{H}}\right)=1$, we see that condition $(\mathrm{H} 1)$ implies that the pair $(\lambda, \omega)$ determines a splitting

$$
T M=\left(\ell_{\omega}, X_{\mathcal{H}}\right) \oplus\left(\xi^{\mathcal{H}}, \omega\right)
$$

of the tangent space of $M$ into a framed line bundle $\left(\ell_{\omega}, X_{\mathcal{H}}\right)$ and a symplectic 2-plane bundle $\left(\xi^{\mathcal{H}}, \omega\right)$.

If we further require that

(H2) $\omega$ is closed

then we can conclude that any section $v \in \Gamma\left(\ell_{\omega}\right)$ of $\ell_{\omega}$, in particular $X_{\mathcal{H}}$, satisfies

$$
L_{v} \omega=i_{v} d \omega+d\left(i_{v} \omega\right)=0
$$

so the flow of any section of $\ell_{\omega}$ preserves $\omega$. Finally, if we require that

(H3) $d \lambda$ vanishes on $\ell_{\omega}$,

then we find that

$$
L_{X_{\mathcal{H}}} \lambda=i_{X_{\mathcal{H}}} d \lambda+d\left(i_{X_{\mathcal{H}}} \lambda\right)=0
$$

so $\lambda$ is preserved by the flow of $X_{\mathcal{H}}$ and hence the splitting (2-1) is also preserved by the flow of $X_{\mathcal{H}}$. Following [5], we will refer to a pair $\mathcal{H}=(\lambda, \omega)$ satisfying $(\mathrm{H} 1)-(\mathrm{H} 3)$ as a stable Hamiltonian structure on $M$. We will refer to the vector field $X_{\mathcal{H}}$ arising from such a structure as the Reeb vector field associated to $\mathcal{H}$, and we will refer the hyperplane bundle $\xi^{\mathcal{H}}$ as the hamiltonian hyperplane field associated to $\mathcal{H}$.

In what follows the dynamics of the vector field $X_{\mathcal{H}}$ will play an important role and the periodic orbits of $X_{\mathcal{H}}$ will be of particular interest. For our purposes, it will be convenient to think of periodic orbits as maps parametrized by $S^{1} \approx \mathbb{R} / \mathbb{Z}$ equipped with the basepoint $0 \in \mathbb{R} / \mathbb{Z}$. More precisely, for $\tau>0$ we define the set $\widetilde{\mathcal{P}}_{\tau}^{0}(M, \mathcal{H})$ of simple $\tau$-periodic orbits of $X_{\mathcal{H}}$ to be the set of all $\gamma \in C^{\infty}\left(S^{1}, M\right)$, such that $\gamma$ is an embedding and $\gamma$ satisfies the equation

$$
d \gamma(t) \partial_{t}=\tau X_{\mathcal{H}}(\gamma(t))
$$


for all $t \in S^{1}$. We will denote the set of all simple periodic orbits by

$$
\widetilde{\mathcal{P}}^{0}(M, \mathcal{H}):=\cup_{\tau>0} \widetilde{\mathcal{P}}_{\tau}^{0}(M, \mathcal{H}) .
$$

We note that each set $\widetilde{\mathcal{P}}_{\tau}^{0}(M, \mathcal{H})$ is invariant under the $S^{1}$-action on $C^{\infty}\left(S^{1}, M\right)$ defined by $c * \gamma(t)=\gamma(t+c)$ for $c \in \mathbb{R} / \mathbb{Z}$ and $\gamma \in C^{\infty}\left(S^{1}, M\right)$. We define the space of unparametrized simple periodic orbits

$$
\mathcal{P}^{0}(M, \mathcal{H})=\widetilde{\mathcal{P}}^{0}(M, \mathcal{H}) / S^{1}
$$

to be the space of $S^{1}$-orbits in $\widetilde{\mathcal{P}}^{0}(M, \mathcal{H})$. For some of the results we present (particularly the asymptotic results in Section 3.1.3) the choice of basepoint $0 \in \mathbb{R} / \mathbb{Z}$ will be important for precise statements. Otherwise, we will generally want to think of two simple orbits as the same if they belong to the same class in $\mathcal{P}^{0}(M, \mathcal{H})=\widetilde{\mathcal{P}}^{0}(M, \mathcal{H}) / S^{1}$. In what follows, we will use the same notation for an orbit $\gamma \in \widetilde{\mathcal{P}}^{0}(M, \mathcal{H})$ and its equivalence class in $\mathcal{P}^{0}(M, \mathcal{H})$ and if we write $\gamma_{2}=\gamma_{1}$ for two simple periodic orbits, the "=" should be interpreted modulo the $S^{1}$-action on $\widetilde{\mathcal{P}}^{0}(M, \mathcal{H})$ unless otherwise stated.

Given a $\gamma \in \widetilde{\mathcal{P}}^{0}(M, \mathcal{H})$, we define for any $m \in \mathbb{Z} \backslash\{0\}$ a map $\gamma^{m} \in C^{\infty}\left(S^{1}, M\right)$ by

$$
\gamma^{m}=\gamma \circ p_{m}
$$

where $p_{m}: S^{1} \rightarrow S^{1}$ is the $m$-fold covering map defined by $p_{m}\left([t]_{\mathbb{R} / \mathbb{Z}}\right)=[m t]_{\mathbb{R} / \mathbb{Z}}$. We refer to $\gamma^{m}$ as a multiply covered periodic orbit with multiplicity $m$ and we denote the set of all periodic orbits (simple and multiply covered) by

$$
\mathcal{P}(M, \mathcal{H}) \text {. }
$$

Let $\psi: \mathbb{R} \times M \rightarrow M$ be the flow of the $X_{\mathcal{H}}$, ie

$$
\dot{\psi}_{t}(x)=X_{\mathcal{H}}\left(\psi_{t}(x)\right)
$$

for all $(t, x) \in \mathbb{R} \times M$. If $\gamma \in \widetilde{\mathcal{P}}^{0}(M, \mathcal{H})$ is a simple $T$-periodic orbit, then it follows from the fact that $L_{X_{\mathcal{H}}} \lambda=0$ and $L_{X_{\mathcal{H}}} \omega=0$ observed earlier that $\left.d \psi_{m T}\right|_{\xi_{\mathcal{\gamma}(0)}^{\mathcal{H}}} \in$ $S p\left(\xi_{\gamma(0)}^{\mathcal{H}}, \omega_{\gamma(0)}\right)$ for all $m \in \mathbb{Z}$. We say that the periodic orbit $\gamma^{m}$ is nondegenerate if $\left.d \psi_{m T}\right|_{\xi_{\gamma(0)}^{\mathcal{H}}}$ does not have 1 in its spectrum. We will say a Hamiltonian structure $\mathcal{H}=(\lambda, \omega)$ is nondegenerate if all periodic orbits of the corresponding vector field $X_{\mathcal{H}}$ are nondegenerate. If $\gamma^{m}$ is nondegenerate we say that

- $\gamma^{m}$ is hyperbolic if $\left.d \psi_{m T}\right|_{\xi_{\gamma(0)}^{\mathcal{H}}}$ has real eigenvalues and that

- $\gamma^{m}$ is elliptic if $\left.d \psi_{m T}\right|_{\xi_{\gamma_{(0)}}^{\mathcal{H}}}$ has complex eigenvalues. 
We will furthermore say that

- $\gamma^{m}$ is even if it is hyperbolic and $\left.d \psi_{m T}\right|_{\xi_{\mathcal{\gamma}(0)}^{\mathcal{H}}}$ has positive eigenvalues, and

- $\gamma^{m}$ is odd if it is either elliptic or if it is hyperbolic and $\left.d \psi_{m T}\right|_{\xi_{\gamma(0)}^{\mathcal{H}}}$ has negative eigenvalues.

The designation of a periodic orbit as even or odd will correspond to the parity of the Conley-Zehnder index of that orbit (see Section 3.1.1).

\subsection{Almost complex cobordisms}

Let $(M, \mathcal{H})$ be a manifold equipped with a stable Hamiltonian structure $\mathcal{H}=(\lambda, \omega)$. We would like to define a preferred class of almost complex structures on $\mathbb{R} \times M$ which interact in a specific way with the Hamiltonian structure. First recall that given any symplectic vector bundle $(E, \omega)$, a complex structure $J$ on $E$ is said to be compatible with $\omega$ if the bilinear form defined by

$$
g_{J}(\cdot, \cdot)=\omega(\cdot, J \cdot)
$$

is a metric on $E$. It is a well know fact that the space of all such $J$ is nonempty and contractible in the $C^{\infty}$ topology (see eg Hofer and Zehnder [13, Section 1.3, Proposition 5 and the discussion which follows]).

Recalling now that $\left(\xi^{\mathcal{H}}, \omega\right)$ is a symplectic vector bundle, we define the set $\mathcal{J}(M, \mathcal{H})$ to be the set of complex structures on $\xi^{\mathcal{H}}$ which are compatible with $\omega$. Given a $J \in \mathcal{J}(M, \mathcal{H})$, we can extend it to an $\mathbb{R}$-invariant almost complex structure $\widetilde{J}$ on $\mathbb{R} \times M$ by requiring

$$
\widetilde{J} \partial_{a}=X_{\mathcal{H}} \quad \text { and }\left.\quad \widetilde{J}\right|_{\xi^{\mathcal{H}}}=J
$$

where $a$ is the parameter along $\mathbb{R}$. We will refer to the almost complex structure $\widetilde{J}$ on $\mathbb{R} \times M$ defined in this way as the standard cylindrical almost complex structure associated to $J \in \mathcal{J}(M, \mathcal{H})$.

Let $W$ be a 4-manifold without boundary. We define a positive Hamiltonian structured end to be data $\widetilde{E}^{+}=\left(E^{+}, \Phi^{+}, M^{+}, \mathcal{H}^{+}\right)$where $E^{+} \subset W$ is an open subset of $W$, $M^{+}$is a closed (possible disconnected) manifold equipped with a stable Hamiltonian structure $\mathcal{H}^{+}$and $\Phi^{+}: E^{+} \rightarrow \mathbb{R}^{+} \times M^{+}$is a diffeomorphism. Similarly we will define a negative Hamiltonian structured end to be data $\widetilde{E}^{-}=\left(E^{-}, \Phi^{-}, M^{-}, \mathcal{H}^{-}\right)$ where everything is as before except that $\Phi^{-}$is now a diffeomorphism mapping $E^{-}$ to $\mathbb{R}^{-} \times M^{-}$. A 4-manifold $W$ equipped with (possibly empty) cylindrical ends $\widetilde{E}^{ \pm}=\left(E^{ \pm}, \Phi^{ \pm}, M^{ \pm}, \mathcal{H}^{ \pm}\right)$will be called a cobordism of Hamiltonian structures or a 
manifold with Hamiltonian structured ends if $W \backslash E^{+} \cup E^{-}$is a compact manifold with (possibly empty) boundary. If a manifold $W$ with Hamiltonian structured ends $\widetilde{E}^{ \pm}=\left(E^{ \pm}, \Phi^{ \pm}, M^{ \pm}, \mathcal{H}^{ \pm}\right)$is equipped with an almost complex structure $\bar{J}$, we say that $\bar{J}$ is compatible with the ends $\widetilde{E}^{ \pm}$if it is conjugated to a standard cylindrical almost complex structure on the ends, that is, if $d \Phi^{ \pm} \circ \bar{J}=\widetilde{J}^{ \pm} \circ d \Phi^{+}$for some $J^{ \pm} \in \mathcal{J}\left(M^{ \pm}, \mathcal{H}^{ \pm}\right)$. We will refer to $(\mathbb{R} \times M, \bar{J})$ as a cylindrical cobordism if $\bar{J}=\widetilde{J}$ is the standard cylindrical almost complex structure associated to $J \in \mathcal{J}(M, \mathcal{H})$ for some Hamiltonian structure $\mathcal{H}$ on $M$. In practice, we will usually suppress the set $E^{ \pm}$ and diffeomorphism $\Phi^{ \pm}$and just refer to the data $\left(\mathbb{R}^{ \pm} \times M^{ \pm}, \mathcal{H}^{ \pm}\right)$as a cylindrical end, or $\left(\mathbb{R}^{ \pm} \times M^{ \pm}, \mathcal{H}^{ \pm}, J^{ \pm}\right)$when we wish to specify the almost complex structure on the ends.

Now, let $\left(W_{1}, \bar{J}_{1}\right)$ and $\left(W_{2}, \bar{J}_{2}\right)$ be cobordisms of Hamiltonians structures equipped with compatible almost complex structures and assume that $W_{1}$ is equipped with negative cylindrical end $\left(\mathbb{R}^{-} \times M_{1}, \mathcal{H}_{1}, J_{1}\right)$ and that $W_{2}$ is equipped with positive cylindrical end $\left(\mathbb{R}^{+} \times M_{2}, \mathcal{H}_{2}, J_{2}\right)$. We say that $\left(W_{1}, \bar{J}_{1}\right)$ can be stacked on $\left(W_{2}, \bar{J}_{2}\right)$ if $M_{1}=M_{2}=M, \mathcal{H}_{1}=\mathcal{H}_{2}$ and $J_{1}=J_{2}$. In this case, we define the concatenation $W_{1} \odot W_{2}$ of $W_{1}$ and $W_{2}$ to be the $C^{0}$-manifold obtained by compactifying the negative end of $W_{1}$ with $\{-\infty\} \times M$, compactifying the positive end of $W_{2}$ with $\{+\infty\} \times M$ and making the obvious identification of $\{-\infty\} \times M$ with $\{+\infty\} \times M$. This operation can obviously be generalized to an arbitrarily long list of cobordisms $\left\{\left(W_{1}, \bar{J}_{1}\right),\left(W_{2}, \bar{J}_{2}\right), \ldots,\left(W_{N}, \bar{J}_{N}\right)\right\}$ provided of course that the negative end of $W_{i}$ matches with the positive end of $W_{i+1}$.

We remark that in the setting of symplectic field theory, the cobordisms considered are usually equipped with a symplectic form and it is assumed that the almost complex structure is compatible with the symplectic form. In some cases, these conditions allow one to obtain topological controls on energy and this control in turn is important for compactness theorems necessary to define the algebraic structure of the theory. However, here we are primarily concerned with intersection-theory-related algebraic invariants which only require an almost complex structure with the correct asymptotic behavior. Therefore, we will not make any assumptions about the behavior of the almost complex structure away from the cylindrical ends.

\subsection{Asymptotically cylindrical maps and pseudoholomorphic curves}

Let $(M, \mathcal{H}=(\lambda, \omega))$ be a closed manifold equipped with a stable Hamiltonian structure and let $\gamma \in \widetilde{\mathcal{P}}^{0}(M, \mathcal{H})$ be a simple $\tau$-periodic orbit of $X_{\mathcal{H}}$. For some $m \in \mathbb{Z} \backslash\{0\}$, consider the map

$$
\tilde{\gamma}^{m}: \mathbb{R} \times S^{1} \rightarrow \mathbb{R} \times M
$$


defined by

$$
\tilde{\gamma}^{m}(s, t)=\left(m \tau s, \gamma^{m}(t)\right) \in \mathbb{R} \times M .
$$

We will refer to such a map as a cylinder over the periodic orbit $\gamma^{m}$ or simply as an orbit cylinder. The reader should note that for any $J \in \mathcal{J}(M, \mathcal{H})$, orbit cylinders have $\widetilde{J}$-invariant tangent spaces.

In this paper, the main objects we will study are maps from a punctured Riemann surface to a cobordism of Hamiltonian structures with the maps asymptotic at the punctures to orbit cylinders. More precisely, we consider a quadruple $(\Sigma, j, \Gamma, \tilde{u})$ where $(\Sigma, j)$ is a closed Riemann surface, $\Gamma \subset \Sigma$ is a finite set and

$$
\tilde{u}=(a, u): \Sigma \backslash \Gamma \rightarrow \mathbb{R} \times M
$$

is a smooth map. We say that $\tilde{u}$ is asymptotically cylindrical over $\gamma^{m}$ at $z_{*} \in \Gamma$ if there exists a holomorphic embedding

$$
\phi:[R, \infty) \times S^{1} \subset \mathbb{C} / i \mathbb{Z} \rightarrow \Sigma \backslash \Gamma
$$

satisfying $\lim _{s \rightarrow \infty} \phi(s, t)=z_{*}$ so that the maps

$$
\tilde{v}_{c}:[R, \infty) \times S^{1} \rightarrow \mathbb{R} \times M
$$

defined by

$$
\tilde{v}_{c}:=(a(\phi(s+c, t))-m \tau c, u(\phi(s+c, t)))
$$

satisfy

$$
\lim _{c \rightarrow \infty} \tilde{v}_{c}=\left.\tilde{\gamma}^{m}\right|_{[R, \infty) \times S^{1}} \text { in } C^{1}\left([R, \infty) \times S^{1}, \mathbb{R} \times M\right) .
$$

The map $\tilde{u}$ is said to be a smooth asymptotically cylindrical map if $\tilde{u}$ is asymptotically cylindrical at each $z \in \Gamma$ over some periodic orbit $\gamma_{z}^{m_{z}}$. In this case we will say that $\gamma_{z}^{m_{z}}$ is the asymptotic limit of $\tilde{u}$ at $z$. Note that if $m_{z}>0$ (resp. $<0$ ) then the $\mathbb{R}$-component of $\tilde{u}$ approaches $+\infty$ (resp. $-\infty$ ) near $z$. If $m_{z}>0$, we will refer to $z$ as a positive puncture of $\tilde{u}$ and similarly, if $m_{z}<0$, we will refer to $z$ as a negative puncture of $\tilde{u}$. When convenient we will write $\Gamma=\Gamma_{+} \cup \Gamma_{-}$to indicate how $\Gamma$ decomposes as positive punctures, $\Gamma_{+}$and negative punctures $\Gamma_{-}$.

We will denote the space of smooth asymptotically cylindrical maps in $\mathbb{R} \times M$ from a genus $g$ surface with $n$ punctures by

$$
C_{g, n}^{\infty}(M, \mathcal{H})
$$

and we will let

$$
C^{\infty}(M, \mathcal{H}):=\cup_{g>0} \cup_{n \geq 0} C_{g, n}^{\infty}(M, \mathcal{H})
$$

denote the space of all smooth asymptotically cylindrical maps in $\mathbb{R} \times M$. 
In a similar manner, we can define asymptotically cylindrical maps in a 4-manifold $W$ with Hamiltonian structured ends $E^{ \pm}=\left(\mathbb{R}^{ \pm} \times M^{ \pm}, \mathcal{H}^{ \pm}\right)$. Let $\tilde{u}: \Sigma \backslash \Gamma \rightarrow W$ be a smooth map and assume that each $z \in \Gamma$ has an open neighborhood $U_{z} \subset \Sigma$ so that the image $\tilde{u}\left(U_{z} \backslash\{z\}\right)$ of the punctured neighborhood lies entirely within one of the cylindrical ends $E^{+}$or $E^{-}$. Then we can think of the map $\left.\tilde{u}\right|_{U_{z} \backslash\{z\}}$ as a map to $\mathbb{R} \times M^{ \pm}$and define what it means for $\tilde{u}$ to be asymptotically cylindrical over a periodic orbit as we did above. The map $\tilde{u}$ is then said to be asymptotically cylindrical if it is asymptotically cylindrical at each $z \in \Gamma$ over some periodic orbit $\gamma_{z}^{m_{z}}$. For asymptotically cylindrical maps in a cobordism, the punctures approaching periodic orbits $\gamma_{z}^{m_{z}}$ in the positive cylindrical end $\mathbb{R}^{+} \times M^{+}$are always positive punctures (ie have $m_{z}>0$ ) and similarly those punctures at which $\tilde{u}$ approaches a periodic orbit $\gamma_{z}^{m_{z}}$ in the negative end $\mathbb{R}^{-} \times M^{-}$have $m_{z}<0$. We will denote the space of genus $g, n$-punctured asymptotically cylindrical maps in $W$ by

$$
C_{g, n}^{\infty}\left(W, \mathcal{H}^{+}, \mathcal{H}^{-}\right)
$$

and the space of all asymptotically cylindrical maps in $W$ by

$$
C^{\infty}\left(W, \mathcal{H}^{+}, \mathcal{H}^{-}\right)
$$

Now, let $\left(W_{1}, \bar{J}_{1}\right)$ and $\left(W_{2}, \bar{J}_{2}\right)$ be almost complex cobordisms and assume that $W_{1}$ is equipped with negative cylindrical end $\left(\mathbb{R}^{-} \times M, \mathcal{H}, J\right)$ and that $W_{2}$ is equipped with positive cylindrical end $\left(\mathbb{R}^{+} \times M, \mathcal{H}, J\right)$ so that we can form the concatenation $W_{1} \odot W_{2}$. For $i \in\{1,2\}$, let

$$
\tilde{u}_{i}: \Sigma_{i} \backslash\left(\Gamma_{i,+} \cup \Gamma_{i,-}\right) \rightarrow W_{i}
$$

be asymptotically cylindrical maps. Assume that there exists a bijection

$$
i: \Gamma_{2,+} \rightarrow \Gamma_{1,-}
$$

so that if $\tilde{u}_{2}$ as asymptotic at $z \in \Gamma_{2,+}$ to $\gamma^{m}$, then $\tilde{u}_{1}$ is asymptotic at $i(z) \in \Gamma_{1,-}$ to $\gamma^{-m}$. Then we can form the concatenated map

$$
\tilde{u}_{1} \odot \tilde{u}_{2}: \Sigma_{1} \odot \Sigma_{2} \backslash\left(\Gamma_{1}^{+} \cup \Gamma_{2}^{-}\right) \rightarrow W_{1} \odot W_{2}
$$

where $\Sigma_{1} \odot \Sigma_{2}$ is the topological surface formed by circle compactifying $\Sigma_{1}$ at its negative punctures, circle compactifying $\Sigma_{2}$ at its positive punctures and then identifying each negative circle with its corresponding (under the bijection $i$ ) positive circle in a way that makes $\tilde{u}_{1} \odot \tilde{u}_{2}$ a continuous map. Note that when the asymptotic data contains multiply covered orbits, $\Sigma_{1} \odot \Sigma_{2}$ is only well-defined up to Dehn twists unless further choices (namely so-called asymptotic markers) are made. The specifics won't be important here, so we won't address this issue any further. We will refer to a 
map $\tilde{u}_{1} \odot \tilde{u}_{2}$ constructed in this way as a smooth asymptotically cylindrical building in $W_{1} \odot W_{2}$.

Consider an asymptotically cylindrical map $(\Sigma, j, \Gamma, \tilde{u}) \in C^{\infty}\left(W, \mathcal{H}^{+}, \mathcal{H}^{-}\right)$and assume that $W$ is equipped with an almost complex structure $\bar{J}$ compatible with the cylindrical ends. If the map $\tilde{u}$ satisfies the equation

$$
d \tilde{u} \circ j=\bar{J} \circ d \tilde{u}
$$

we say that $(\Sigma, j, \Gamma, \tilde{u})$ is an asymptotically cylindrical pseudoholomorphic map. We define an equivalence relation on punctured pseudoholomorphic maps by saying that $(\Sigma, j, \Gamma, \tilde{u})$ is equivalent to $\left(\Sigma^{\prime}, j^{\prime}, \Gamma^{\prime}, \tilde{u}^{\prime}\right)$ if there exists a biholomorphic map

$$
\phi: \Sigma^{\prime} \rightarrow \Sigma
$$

so that $\Gamma=\phi\left(\Gamma^{\prime}\right)$ and $\tilde{u} \circ \phi=\tilde{u}^{\prime}$. An equivalence class of maps $[\Sigma, j, \Gamma, u]$ will be referred to as an asymptotically cylindrical pseudoholomorphic curve. We will use the notations

$$
\mathcal{M}\left(W, \bar{J}, \mathcal{H}^{+}, \mathcal{H}^{-}\right) \text {and } \mathcal{M}(M, \mathcal{H}, J)
$$

to denote the set of asymptotically cylindrical pseudoholomorphic curves in $(W, \bar{J})$ or $(\mathbb{R} \times M, \widetilde{J})$ respectively, and similarly we will use

$$
\mathcal{M}_{g, n}\left(W, \bar{J}, \mathcal{H}^{+}, \mathcal{H}^{-}\right) \text {and } \mathcal{M}_{g, n}(M, \mathcal{H}, J) .
$$

if we wish to specify the genus and number of punctures.

Note To simplify our language, we will henceforth simply use the term "pseudoholomorphic curve/map" when we are referring to asymptotically cylindrical pseudoholomorphic curves/maps. The reader should always assume that all pseudoholomorphic curves are asymptotically cylindrical unless stated otherwise.

As with smooth asymptotically cylindrical maps, we can concatenate pairs (or finite lists) of asymptotically cylindrical pseudoholomorphic maps into cobordisms with matching ends, providing the asymptotic data match appropriately. Following [2], we will call such maps pseudoholomorphic buildings.

\subsection{Main results}

Throughout this section, we will let $(M, \mathcal{H}=(\lambda, \omega))$ denote a 3-manifold equipped with a nondegenerate stable Hamiltonian structure and we will let $(W, \bar{J})$ denote an almost complex 4-manifold equipped with cylindrical ends $E^{ \pm}=\left(\mathbb{R}^{ \pm} \times M^{ \pm}, \mathcal{H}^{ \pm}, J^{ \pm}\right)$ where the stable Hamiltonian structures $\mathcal{H}^{ \pm}$are assumed to be nondegenerate. 
The main results of this paper are concerned with the intersection properties of pseudoholomorphic curves in 4-manifolds with Hamiltonian structured cylindrical ends. Due to the noncompactness of the domains of the curves we consider, the problem of understanding intersection behavior becomes particularly subtle in the case that the curves have multiple ends which approach coverings of the same orbit or - when considering self-intersection problems - in the case that an end approaches a multiple cover of an orbit. Indeed, while all intersections (or self-intersections and singularities) must be isolated by the results of Micallef and White [18], it is not immediately obvious why the algebraic intersection number of two curves - computed by summing local intersection indices - must be finite since it is conceivable that a pair of curves could have a sequence of intersections approaching the punctures. Similarly, it is not clear that a single curve couldn't have a sequence of self-intersections approaching a puncture at which the curve is asymptotic to a multiple cover of an orbit or approaching a pair of punctures at which the curve is asymptotic to coverings of the same orbit. While we will see that the asymptotic results of [22] imply that the intersection number of two curves or the self-intersection index of a single curve must in fact be finite (see Corollary 3.10 and Corollary 3.11 below), an additional unavoidable complication is that these quantities may not be stable under homotopies since intersections could run in or out of the punctures at shared asymptotic limits. We thus seek to determine to what degree these quantities are topologically controlled by the homotopy classes of the maps in $C^{\infty}(M, \mathcal{H})$ or $C^{\infty}\left(W, \mathcal{H}^{+}, \mathcal{H}^{-}\right)$(depending on the target manifold).

As a first step towards finding intersection-related topological invariants of a pair of maps with common asymptotic limits, we perturb one of the maps in a prescribed direction near the ends. This idea was studied locally for a single embedded pseudoholomorphic half-cylinder by Kriener [16] and further pursued by Hutchings [14; 15] and the author [21]. More precisely, let $\Phi$ denote a choice of trivialization of the plane-field $\xi^{\mathcal{H}}$ along every simple periodic orbit. Then given two maps $\tilde{u}, \tilde{v} \in C^{\infty}\left(W, \bar{J}, \mathcal{H}^{+}, \mathcal{H}^{-}\right)$, we define the relative intersection number $i^{\Phi}(\tilde{u}, \tilde{v})$ of $\tilde{u}$ and $\tilde{v}$ by

$$
i^{\Phi}(\tilde{u}, \tilde{v})=\operatorname{int}\left(\tilde{u}, \tilde{v}_{\Phi}\right)
$$

where $\tilde{v}_{\Phi}$ is the map obtained by perturbing $\tilde{v}$ near the punctures in a direction determined by the trivialization $\Phi$ and where "int" denotes the algebraic intersection number computed by perturbing $\tilde{u}$ and $\tilde{v}_{\Phi}$ on compact subsets of their domain so that they are transverse and then counting intersections with sign. It is well known that the resulting count is independent of the compactly supported perturbation of $\tilde{u}$ and $\tilde{v}_{\Phi}$ since $\tilde{u}$ and $\tilde{v}_{\Phi}$ are disjoint outside of a compact set. Thus the relative intersection number computed in this way depends on the homotopy classes of $\tilde{u}$ and $\tilde{v}$ in $C^{\infty}\left(W, \bar{J}, \mathcal{H}^{+}, \mathcal{H}^{-}\right)$and the homotopy class of the trivialization $\Phi$. 
While the relative intersection number gives a topological invariant of a pair of asymptotically cylindrical maps, its relationship to the algebraic intersection number of two (unperturbed) pseudoholomorphic curves is not clear and in particular it is not immediately clear whether or how it can be used to find a bound on the algebraic intersection number of two pseudoholomorphic curves. We will see below that through careful consideration of the relative asymptotic behavior of the curves from [22], such a bound depending only on the homotopy classes of the maps can be obtained by adding an appropriate quantity to the relative intersection number which balances the trivialization dependence. Motivated by the local analysis in Section 3, we define the generalized intersection number $[\tilde{u}] *[\tilde{v}]$ of two asymptotically cylindrical maps $(\Sigma, j, \Gamma, \tilde{u})$, $\left(\Sigma^{\prime}, j^{\prime}, \Gamma^{\prime}, \tilde{v}\right) \in C^{\infty}\left(W, \mathcal{H}^{+}, \mathcal{H}^{-}\right)$as follows: assuming that $\tilde{u}$ is asymptotic at $z \in \Gamma$ to a cylinder over $\gamma_{z}^{m_{z}}$ and similarly that $\tilde{v}$ is asymptotic at $w \in \Gamma^{\prime}$ to a cylinder over $\gamma_{w}^{m_{w}}$ we define $[\tilde{u}] *[\tilde{v}]$ by

$$
[\tilde{u}] *[\tilde{v}]=i^{\Phi}(\tilde{u}, \tilde{v})+\sum_{\substack{(z, w) \in \Gamma_{z} \times \Gamma^{\prime} \\ \gamma_{z}=\gamma_{w} \\ m_{z} m_{w}>0}} m_{z} m_{w} \max \left\{\frac{\left\lfloor\mu^{\Phi}\left(\gamma_{z}^{m_{z}}\right) / 2\right\rfloor}{\left|m_{z}\right|}, \frac{\left\lfloor\mu^{\Phi}\left(\gamma_{w}^{m_{w}}\right) / 2\right\rfloor}{\left|m_{w}\right|}\right\}
$$

where $\mu^{\Phi}\left(\gamma^{m_{z}}\right)$ denotes the Conley-Zehnder index of the periodic orbit $\gamma_{z}^{m_{z}}$ (see Hofer, Wysocki and Zehnder [8] and Section 3 below) and where $\lfloor\cdot\rfloor$ denotes the greatest integer function. Note that the sum here is taken over all pairs of punctures with the same sign where the maps in question are asymptotically cylindrical over coverings of the same underlying simple (unparametrized) periodic orbit.

The following theorem summarizes the main properties of the generalized intersection number.

Theorem 2.1 (Properties of the generalized intersection number) Let $W, W_{1}$ and $W_{2}$ be 4-manifolds with Hamiltonian structured cylindrical ends and assume we can form the concatenation $W_{1} \odot W_{2}$. Then:

(1) If $(\Sigma, j, \Gamma, \tilde{u})$ and $\left(\Sigma^{\prime}, j^{\prime}, \Gamma^{\prime}, \tilde{v}\right) \in C^{\infty}\left(W, \mathcal{H}^{+}, \mathcal{H}^{-}\right)$are asymptotically cylindrical maps then the generalized intersection number $[\tilde{u}] *[\tilde{v}]$ depends only on the homotopy classes of $\tilde{u}$ and $\tilde{v}$ in $C^{\infty}\left(W, \mathcal{H}^{+}, \mathcal{H}^{-}\right)$.

(2) For any $(\Sigma, j, \Gamma, \tilde{u})$ and $\left(\Sigma^{\prime}, j^{\prime}, \Gamma^{\prime}, \tilde{v}\right) \in C^{\infty}\left(W, \mathcal{H}^{+}, \mathcal{H}^{-}\right)$

$$
[\tilde{u}] *[\tilde{v}]=[\tilde{v}] *[\tilde{u}] .
$$

(3) If $(\Sigma, j, \Gamma, \tilde{u}),\left(\Sigma^{\prime}, j^{\prime}, \Gamma^{\prime}, \tilde{v}\right),\left(\Sigma^{\prime \prime}, j^{\prime \prime}, \Gamma^{\prime \prime}, \tilde{w}\right) \in C^{\infty}\left(W, \mathcal{H}^{+}, \mathcal{H}^{-}\right)$then

$$
[\tilde{u}+\tilde{v}] *[\tilde{w}]=[\tilde{u}] *[\tilde{w}]+[\tilde{v}] *[\tilde{w}]
$$

where "+" on the left hand side denotes the disjoint union of the maps $\tilde{u}$ and $\tilde{v}$. 
(4) If $\tilde{u}_{1} \odot \tilde{u}_{2}$ and $\tilde{v}_{1} \odot \tilde{v}_{2}$ are asymptotically cylindrical buildings in $W_{1} \odot W_{2}$ then

$$
\left[\tilde{u}_{1} \odot \tilde{u}_{2}\right] *\left[\tilde{v}_{1} \odot \tilde{v}_{2}\right] \geq\left[\tilde{u}_{1}\right] *\left[\tilde{v}_{1}\right]+\left[\tilde{u}_{2}\right] *\left[\tilde{v}_{2}\right] .
$$

Moreover, strict inequality occurs if and only if there is a periodic orbit $\gamma$ so that $\tilde{u}_{1}$ has a negative puncture asymptotic to $\gamma^{m}, \tilde{v}_{1}$ has a negative puncture asymptotic to $\gamma^{n}$ and both $\gamma^{m}$ and $\gamma^{n}$ are odd orbits.

In item (4) above, the possibility of strict inequality has to do with the fact that at an odd orbit (with a fixed multiplicity) the eigenvectors of the asymptotic operator controlling the direction of approach of negative pseudoholomorphic ends must have strictly greater winding (computed relative to the direction of the Reeb flow) than those controlling the direction of approach of positive pseudoholomorphic ends. The relevant details from Hofer, Wysocki and Zehnder [10; 8] are reviewed in Section 3.1 below. In some applications it is convenient to modify the generalized intersection number to include information about shared odd orbits so that one has a product which is level-wise additive, ie always satisfies equality in item (4) above. This approach is taken by Momin [19] to study a variation on contact homology where only curves contained in the complement of a prescribed collection of elliptic orbits are included in the differential.

One of the motivations for defining the generalized intersection number as we did is the next theorem, which generalizes the fact that two closed curves without common components have a nonnegative homological intersection number and that the intersection number vanishes only if the two curves do not intersect. The total asymptotic intersection index $\delta_{\infty}(\tilde{u}, \tilde{v})$ mentioned in the theorem is defined in Section 3 below. For the moment the reader should know that it is a nonnegative quantity defined for pairs of pseudoholomorphic curves having no common components that can be thought of as a measure of the degree of tangency at infinity between the two curves. Here we say the two maps $\tilde{u}$ and $\tilde{v}$ have no common components if there is no component of the domain of $\tilde{u}$ which has image identical to that of a component of the domain of $\tilde{v}$.

Theorem 2.2 Let $\left(W^{4}, \bar{J}\right)$ be an almost complex cobordism with cylindrical ends $\left(\mathbb{R}^{ \pm} \times M^{ \pm}, J^{ \pm}, \mathcal{H}^{ \pm}\right)$and let $[\Sigma, j, \Gamma, \tilde{u}],\left[\Sigma^{\prime}, j^{\prime}, \Gamma^{\prime}, \tilde{v}\right] \in \mathcal{M}\left(W, \bar{J}, \mathcal{H}^{+}, \mathcal{H}^{-}\right)$be pseudoholomorphic curves in $W$ with no common components. Then

$$
[\tilde{u}] *[\tilde{v}]=\operatorname{int}(\tilde{u}, \tilde{v})+\delta_{\infty}(\tilde{u}, \tilde{v}),
$$

where $\operatorname{int}(\tilde{u}, \tilde{v})$ is the algebraic intersection number of $\tilde{u}$ and $\tilde{v}$ and $\delta_{\infty}(\tilde{u}, \tilde{v})$ is the asymptotic intersection index of $\tilde{u}$ and $\tilde{v}$. In particular

$$
[\tilde{u}] *[\tilde{v}] \geq \operatorname{int}(\tilde{u}, \tilde{v}) \geq 0,
$$


and

$$
[\tilde{u}] *[\tilde{v}]=0
$$

if and only if $\tilde{u}$ and $\tilde{v}$ do not intersect and the total asymptotic intersection index vanishes, ie $\delta_{\infty}(\tilde{u}, \tilde{v})=0$.

As an immediate corollary to this, we can conclude that if a homotopy class of maps with connected domains in $C^{\infty}\left(W, \mathcal{H}^{+}, \mathcal{H}^{-}\right)$has a negative generalized self-intersection number, then that homotopy class can contain at most one pseudoholomorphic curve for a given compatible almost complex structure.

We note that in contrast to the case of closed pseudoholomorphic curves, positivity of the generalized intersection number of two given curves does not guarantee that the two curves intersect. This is because the algebraic intersection number of two punctured curves is not a homotopy-invariant quantity and intersections between two pseudoholomorphic curves can disappear out the punctures. The degree to which intersections can escape or appear at the punctures is however topologically controlled: even though both quantities on the right hand side of (2-4) can vary under homotopies of curves, their sum is topologically determined and this fact coupled with the nonnegativity of those quantities allows a topological bound on the total count of intersections and "tangencies at infinity." One might consider this fungibility of intersections and "tangencies at infinity" as motivation to think of tangencies at infinity or "asymptotic intersections," as being somehow equivalent to actual intersections. This viewpoint is further supported by the fact that considering weighted Fredholm theory arguments (Hofer, Wysocki and Zehnder [11] and Dragnev [3]) one would expect that the space of pairs of curves $(\tilde{u}, \tilde{v})$ with $\delta_{\infty}(\tilde{u}, \tilde{v})>0$ should have positive codimension in the universal moduli space of pseudoholomorphic curves.

We next state a generalization for punctured curves of the adjunction formula (1-1). We first establish some notation and terminology. Let $(\Sigma, j, \Gamma, \tilde{u}) \in C^{\infty}\left(W, \mathcal{H}^{+}, \mathcal{H}^{-}\right)$be an asymptotically cylindrical map and assume that at $z \in \Gamma, u$ is asymptotic to the periodic orbit $\gamma_{z}^{m_{z}}$. A choice of complex trivialization of the hamiltonian plane-field distribution $\left(\xi^{\mathcal{H}^{ \pm}}, J^{ \pm}\right)$along each $\gamma_{z}$ induces a complex trivialization of the pull-back bundle $\left(\tilde{u}^{*} T W, \bar{J}\right)$ since on the cylindrical ends we have

$$
(T W, \bar{J}) \approx\left(\mathbb{R} \oplus \mathbb{R} X_{\mathcal{H}^{ \pm}} \oplus \xi^{\mathcal{H}^{ \pm}}, \widetilde{J}^{ \pm}\right) \approx \mathbb{C} X_{\mathcal{H}^{ \pm}} \oplus\left(\xi^{\mathcal{H}^{ \pm}}, J^{ \pm}\right) .
$$

Given such a choice of trivialization, $\Phi$, we can define the relative Chern number $c_{1}^{\Phi}\left(\tilde{u}^{*} T W\right)$ which is the obstruction to extending over $\Sigma \backslash \Gamma$ the trivialization of $\left(\tilde{u}^{*} T W, \bar{J}\right) \rightarrow \Sigma \backslash \Gamma$ that has been chosen at the ends (see [14; 21] or Section 4.2.1 
below for a precise definition). We define the total Conley-Zehnder index $\mu(\tilde{u})$ of the map $\tilde{u}$ by

$$
\mu(\tilde{u}):=2 c_{1}^{\Phi}\left(\tilde{u}^{*} T W\right)+\sum_{z \in \Gamma} \mu^{\Phi}\left(\gamma_{z}^{m_{z}}\right) .
$$

It follows from change of trivialization formulas given below for the relative first Chern number and the Conley-Zehnder index that the total Conley-Zehnder index does not depend on the choice of trivialization.

Next, assuming $\gamma$ is a simple periodic orbit of a Reeb vector field we define the spectral covering number of the periodic orbit $\gamma^{m}$ by

$$
\bar{\sigma}\left(\gamma^{m}\right)=\operatorname{gcd}\left(m,\left\lfloor\mu^{\Phi}\left(\gamma^{m}\right) / 2\right\rfloor\right) .
$$

We observe that this quantity does not depend on a choice of trivialization since changing the trivialization changes $\left\lfloor\mu^{\Phi}\left(\gamma^{m}\right) / 2\right\rfloor$ by an integer multiple of $m$ (see comments following Lemma 3.4). With $u$ as in the previous paragraph, we then define the total spectral covering number $\bar{\sigma}(u)$ of $u$ by

$$
\bar{\sigma}(u)=\sum_{z \in \Gamma} \bar{\sigma}\left(\gamma_{z}^{m_{z}}\right)
$$

We observe that the total spectral covering number of a map depends only on its asymptotic limits and not on the map itself.

We now state a generalization of (1-1) involving the generalized intersection number. The quantity $\delta(\tilde{u})$ in equation (2-5), which we call the self-intersection index, is a nonnegative integer-valued quantity which records information about double points and singular points and is zero if and only if $u$ is an embedding (see [18] and Section 4.2.2 below). The asymptotic self-intersection index $\delta_{\infty}(\tilde{u})$ of $\tilde{u}$ is defined in Section 3.2 below. For the moment, the reader should know that it is a nonnegative, integer-valued quantity, defined for a simple pseudoholomorphic curve that can be thought of as a measure of the degree of self-tangency at infinity. Here, we say a pseudoholomorphic curve is simple if it does not factor through a branched cover.

Theorem 2.3 Let $[\Sigma, j, \Gamma, \tilde{u}] \in \mathcal{M}\left(W, \bar{J}, \mathcal{H}^{+}, \mathcal{H}^{-}\right)$be a connected pseudoholomorphic curve and assume that $\tilde{u}$ is simple. Then

$$
[\tilde{u}] *[\tilde{u}]-\frac{1}{2} \mu(\tilde{u})+\frac{1}{2} \# \Gamma_{\text {odd }}+\chi(\Sigma)-\bar{\sigma}(\tilde{u})=2\left[\delta(\tilde{u})+\delta_{\infty}(\tilde{u})\right],
$$

where $\# \Gamma_{\text {odd }}$ is the number of punctures of $\tilde{u}$ with odd Conley-Zehnder indices. In particular,

$$
[\tilde{u}] *[\tilde{u}]-\frac{1}{2} \mu(\tilde{u})+\frac{1}{2} \# \Gamma_{\text {odd }}+\chi(\Sigma)-\bar{\sigma}(\tilde{u}) \geq 0
$$


and equality occurs if and only if $\tilde{u}$ is an embedding and the asymptotic self-intersection index of $\tilde{u}$ vanishes, ie $\delta_{\infty}(\tilde{u})=0$.

Note that in contrast to the case of closed pseudoholomorphic curves and (1-1), positivity of the left-hand side of equation (2-5) does not guarantee a curve is not embedded. This is because the self-intersection index $\delta(\tilde{u})$ is not, in general, topologically determined for punctured curves since double points can escape out the ends in families of curves. However, much like in the discussion following Theorem 2.2 above, this theorem shows that the sum of the self-intersection index and the asymptotic self-intersection index is topologically determined and that double points escaping out the ends are traded for self-tangencies at infinity. Thus, we might think of having a positive asymptotic self-intersection index as being somehow equivalent to having double points or singular points, a point of view which is again supported by the fact that the Fredholm theory suggests that curves with $\delta_{\infty}(\tilde{u})>0$ should form strata of positive codimension in the universal moduli space.

Results closely related to Theorem 2.3 have been proved by Hutchings [14; 15]. Due to the specific application being pursued in those papers, no theorem equivalent to Theorem 2.3 appears; [14, Equation (18) in Remark 3.2] identifies embedded curves, but not in terms of quantities that are topologically determined. Still, the appropriate analogue of that result to the present context is the key step in proving Theorem 2.3. On the other hand, the index inequality in [14, Theorem 1.7] gives a purely topological condition that guarantees a curve will be embedded, but only identifies those curves whose asymptotic data satisfy an additional "admissibility" condition. Theorem 2.3 above, can thus be thought of as being intermediate between these two results.

2.4.1 Cylindrical cobordisms In this section we specialize to the case of a cylindrical cobordism $(\mathbb{R} \times M, \widetilde{J})$ equipped with an $\mathbb{R}$-invariant almost complex structure $\widetilde{J}$ arising from a compatible $J \in \mathcal{J}(M, \mathcal{H})$ for some nondegenerate stable Hamiltonian structure $\mathcal{H}=(\lambda, \omega)$ on $M$.

An important (and well-known) observation is that space of curves $\mathcal{M}(M, \mathcal{H}, J)$ is equipped with an $\mathbb{R}$-action, defined by translating the $\mathbb{R}$-coordinate of the given curve. More precisely, if $[\Sigma, j, \Gamma, \tilde{u}]$ is a pseudoholomorphic curve and we write $\tilde{u}=(a, u) \in \mathbb{R} \times M$, then $\left[\Sigma, j, \Gamma, \tilde{u}_{c}\right]$ where $\tilde{u}_{c}(z):=(a(z)+c, u(z))$ is also a pseudoholomorphic curve. Furthermore, in the cylindrical case, the way the almost complex structure is defined allows the pseudoholomorphic curve equation (2-2) for the map $(\Sigma, j, \Gamma,(a, u))$ to be rewritten,

$$
\begin{gathered}
u^{*} \lambda \circ j=d a \\
\pi_{\xi^{\mathcal{H}}} \circ d u \circ j=J \circ \pi_{\xi^{\mathcal{H}}} \circ d u
\end{gathered}
$$


where $\pi_{\xi \mathcal{H}}: T M=\mathbb{R} X_{\mathcal{H}} \oplus \xi^{\mathcal{H}} \rightarrow \xi^{\mathcal{H}}$ is the projection of $T M$ onto $\xi^{\mathcal{H}}$ determined by the splitting (2-1). Thus, the $M$-component, $u$, of a curve in $\mathbb{R} \times M$ determines the $\mathbb{R}$-component, $a$, up to a constant.

As an immediate consequence of the existence of the $\mathbb{R}$-action, Theorem 2.2 and the homotopy invariance of the generalized intersection number, we can conclude that if a connected curve $[\Sigma, j, \Gamma, \tilde{u}]$ has no component whose image lies in an orbit cylinder, then

$$
[\tilde{u}] *[\tilde{u}]=[\tilde{u}] *\left[\tilde{u}_{c}\right] \geq 0
$$

so such a curve must have a nonnegative self-intersection number.

Since, in the cylindrical case, the projection of the curve to the 3-manifold contains all of the information (up to an $\mathbb{R}$-shift), it is not surprising that generalized intersection number of two curves in $\mathbb{R} \times M$ can be computed in terms of 3-dimensional invariants associated to the curves; namely we can state a formula in terms of the intersections between one of the curves with periodic orbits which are asymptotic limits of the other curve and how the ends of the curves wind around the periodic orbits (see Theorem 5.8 below). Moreover, since the formula we give decomposes into a collection of nonnegative terms, this computation then allows one to deduce necessary and sufficient conditions for the generalized intersection number to vanish, which we state in Corollary 5.9.

Part of the reason we seek to find conditions to characterize when the generalized intersection number of two curves $[\Sigma, j, \Gamma, \tilde{u}=(a, u)]$ and $\left[\Sigma^{\prime}, j^{\prime}, \Gamma^{\prime}, \tilde{v}=(b, v)\right] \in$ $\mathcal{M}(M, \mathcal{H}, J)$ is zero (or nonzero) is that $[\tilde{u}] *[\tilde{v}]=0$ implies that the projections $u$, $v$, of the maps to $M$ do not intersect, provided that these maps have no components projecting to identical images in $M$. Indeed, if we can find a $z \in \Sigma$ and $w \in \Sigma^{\prime}$ so that $u(z)=v(w)$ then we can find a $c$ so that $\tilde{u}(z)=\tilde{v}_{c}(w)$, which in turn implies that $\operatorname{int}\left(\tilde{u}, \tilde{v}_{c}\right)>0$ and hence $[\tilde{u}] *[\tilde{v}]=[\tilde{u}] *\left[\tilde{v}_{c}\right] \geq \operatorname{int}\left(\tilde{u}, \tilde{v}_{c}\right)>0$ by Theorem 2.2. Thus the vanishing of the generalized intersection number gives a sufficient condition for the projection of the curves to the three-manifold to not intersect. The vanishing of the generalized intersection number is not a necessary condition for the projected curves to not intersect since it is possible for the algebraic intersection number of $\tilde{u}$ and $\tilde{v}_{c}$ to be zero for all $c \in \mathbb{R}$, but still have $[\tilde{u}] *[\tilde{v}]>0$, since the asymptotic intersection index could be nonzero. However, in the $\mathbb{R}$-invariant setting, the asymptotic intersection number changes in a predictable manner when $\mathbb{R}$-shifting one of the two curves and we are able to establish a set of necessary and sufficient conditions for the two projected curves to not intersect.

Before stating the relevant theorem we discuss some of the more immediate necessary conditions for the projected curves $u$ and $v$ to not intersect. We first recall some facts 
from Hofer, Wysocki and Zehnder [10; 8]. Namely, if a curve $[\Sigma, j, \Gamma, \tilde{u}=(a, u)]$ does not have any components with image lying in an orbit cylinder, then there is a neighborhood of each puncture on which the map does not intersect any of its asymptotic limits. Thus choosing a loop in one of these neighborhoods which winds around the puncture once in a clockwise direction, ${ }^{1}$ and choosing a trivialization $\Phi$ of $\xi^{\mathcal{H}}$ along the orbit, we get a well defined winding number wind ${ }_{\infty}^{\Phi}(\tilde{u} ; z)$ for each $z \in \Gamma$. Moreover, it is shown in [8] (and reviewed in Lemma 3.13 below) that

$$
\operatorname{wind}_{\infty}^{\Phi}(\tilde{u} ; z) \leq\left\lfloor\mu^{\Phi}\left(\gamma_{z}^{m_{z}}\right) / 2\right\rfloor
$$

where we assume $\tilde{u}$ to be asymptotic to $\gamma_{z}^{m_{z}}$ at $z \in \Gamma$. If we consider a small torus $T_{\gamma_{z}}^{2}$ bounding a tubular neighborhood of $\gamma_{z}$ and use the framing $\Phi$ to identify $H_{1}\left(T_{\gamma_{z}}^{2}\right)$ with $\mathbb{Z} \oplus \mathbb{Z}$, the intersection of the projected map $u$ with $T_{\gamma_{z}}^{2}$ will be a curve in the homology class $\left(m_{z}, \operatorname{wind}^{\Phi}(\tilde{u} ; z)\right)$. If $\tilde{v}$ also has an end approaching $\gamma_{z}$ at $w \in \Gamma^{\prime}$, the homology class of intersection of $v$ with $T_{\gamma_{z}}^{2}$ will then be given by $\left(m_{w}\right.$, wind $\left.{ }^{\Phi}(\tilde{v} ; w)\right)$. Thus, if the projected maps $u$ and $v$ are to be disjoint, we must have that the intersection number

$$
\left(m_{z}, \operatorname{wind}^{\Phi}(\tilde{u} ; z)\right) \cdot\left(m_{w}, \operatorname{wind}^{\Phi}(\tilde{v} ; w)\right)=m_{z} \operatorname{wind}^{\Phi}(\tilde{v} ; w)-m_{w} \operatorname{wind}^{\Phi}(\tilde{u} ; z)
$$

is zero or equivalently

$$
\frac{\operatorname{wind}_{\infty}^{\Phi}(\tilde{u} ; z)}{m_{z}}=\frac{\operatorname{wind}_{\infty}^{\Phi}(\tilde{v} ; w)}{m_{w}} .
$$

Therefore, if the projections $u$ and $v$ do not intersect, it is necessary that (2-7) holds at any pair of punctures $(z, w) \in \Gamma \times \Gamma^{\prime}$ at which $u$ and $v$ are asymptotic to coverings of a common orbit.

Next suppose that $u$ intersects one of the asymptotic limits of $v$. Then one can use the asymptotic results of [10] (or see Theorem 3.7 below) with the fact that the orbit is a projection to $M$ of a pseudoholomorphic curve in $\mathbb{R} \times M$ to argue that $u$ must intersect $v$. Thus if $u$ and $v$ do not intersect, it must be the case that $u$ intersects none of the asymptotic limits of $v$ and vice versa.

As the following theorem shows, the necessary conditions we have just stated for the projections of two curves to $M$ to not intersect are also sufficient and in fact somewhat weaker conditions are sufficient.

\footnotetext{
${ }^{1}$ This convention agrees with that used in [8] for positive punctures, but is opposite that used in [8] for negative punctures. What this means geometrically is that we compute windings by traversing the orbit in a direction determined by the orientation of the boundary of the $S^{1}$-compactified punctured surface, while in [8] the convention is to compute windings by traversing the orbit in the direction determined by the Reeb vector field. We use this convention because it simplifies the statements of most of our results.
} 
Theorem 2.4 Let $[\Sigma, j, \Gamma, \tilde{u}=(a, u)]$ and $\left[\Sigma^{\prime}, j^{\prime}, \Gamma^{\prime}, \tilde{v}=(b, v)\right] \in \mathcal{M}(M, \mathcal{H}, J)$ be pseudoholomorphic curves and assume that no component of $\tilde{u}$ or $\tilde{v}$ lies in in orbit cylinder and that the projected curves $u$ and $v$ do not have identical image on any component of their domains. Then the following are equivalent:

(1) The projected curves $u$ and $v$ do not intersect.

(2) All of the following hold:

(a) The map $u$ does not intersect any of the positive asymptotic limits of $v$.

(b) The map $v$ does not intersect any of the negative asymptotic limits of $u$.

(c) If $\gamma$ is a periodic orbit so that at $z \in \Gamma, \tilde{u}$ is asymptotic to $\gamma^{m_{z}}$ and at $w \in \Gamma^{\prime}, \tilde{v}$ is asymptotic to $\gamma^{m_{w}}$, then:

(i) If $m_{z}$ and $m_{w}$ have the same sign then

$$
\frac{\operatorname{wind}_{\infty}(\tilde{u} ; z)}{m_{z}} \geq \frac{\operatorname{wind}_{\infty}(\tilde{v} ; w)}{m_{w}} .
$$

(ii) If $m_{z}<0$ and $m_{w}>0$ then

$$
\frac{\operatorname{wind}_{\infty}^{\Phi}(\tilde{u} ; z)}{m_{z}}=\frac{\left\lfloor\mu^{\Phi}\left(\gamma^{m_{z}}\right) / 2\right\rfloor}{m_{z}}=\frac{\left\lfloor\mu^{\Phi}\left(\gamma^{m_{w}}\right) / 2\right\rfloor}{m_{w}}=\frac{\operatorname{wind}_{\infty}^{\Phi}(\tilde{v} ; w)}{m_{w}}
$$

(this is only possible if $\gamma^{m_{z}}$ and $\gamma^{m_{w}}$ are both even orbits).

(3) All of the following hold:

(a) The map $u$ does not intersect any of the asymptotic limits of $v$.

(b) The map $v$ does not intersect any of the asymptotic limits of $u$.

(c) If $\gamma$ is a periodic orbit so that at $z \in \Gamma, \tilde{u}$ is asymptotic to $\gamma^{m_{z}}$ and at $w \in \Gamma^{\prime}, \tilde{v}$ is asymptotic to $\gamma^{m_{w}}$, then

$$
\frac{\operatorname{wind}_{\infty}(\tilde{u} ; z)}{m_{z}}=\frac{\operatorname{wind}_{\infty}(\tilde{v} ; w)}{m_{w}} .
$$

When two curves, $[\Sigma, j, \Gamma, \tilde{u}]$ and $\left[\Sigma^{\prime}, j^{\prime}, \Gamma^{\prime}, \tilde{v}\right] \in \mathcal{M}(M, \mathcal{H}, J)$, each have as an asymptotic limit a cover of the same even periodic orbit, it is sometimes possible to conclude that the generalized intersection number $[\tilde{u}] *[\tilde{v}]$ is positive. Let $\gamma$ denote either a simple, even orbit or the double cover of an odd, hyperbolic orbit. In this case it is possible to use the asymptotic description from Theorem 3.7 below to define a notion of two curves approaching a cover of $\gamma$ in the same direction. We delay the precise definition to Section 5.3.1 because it is somewhat technical. The following theorem then says that two curves approaching a cover of $\gamma$ in the same direction is a sufficient condition for a positive generalized intersection number.

Theorem 2.5 Let $\gamma$ be a periodic orbit satisfying the above assumptions and let $[\Sigma, j, \Gamma, \tilde{u}=(a, u)]$ and $\left[\Sigma^{\prime}, j^{\prime}, \Gamma^{\prime}, \tilde{v}=(b, v)\right] \in \mathcal{M}(M, \mathcal{H}, J)$ be connected pseudoholomorphic curves. Assume that at punctures $z \in \Gamma$ and $w \in \Gamma^{\prime}, \tilde{u}$ and $\tilde{v}$ approach a 
cover of $\gamma$ in the same direction, and that there do not exist neighborhoods $U$ of $z$ and $V$ of $w$ so that $u(U \backslash\{z\})=v(V \backslash\{w\})$. Then

$$
[\tilde{u}] *[\tilde{v}]>0 .
$$

Just as it is of use to know when the generalized intersection number of a pair of curves vanishes, it is of use to know when the generalized self-intersection number of a single curve $[\Sigma, j, \Gamma, \tilde{u}=(a, u)] \in \mathcal{M}(M, \mathcal{H}, J)$ vanishes. Combining the adjunction formula (2-5) with the previously referenced formula (5-5) for the generalized intersection number in terms of data associated to the projected curves, we get a collection of conditions equivalent to the vanishing of the generalized self-intersection number $[\tilde{u}] *[\tilde{u}]$, provided the map $\tilde{u}$ is simple and does not have any components contained in an orbit cylinder (Corollary 5.17 below). From this result we will see that under the stated assumptions, $[\tilde{u}] *[\tilde{u}]=0$ implies that $\tilde{u}$ is an embedding which projects to an immersion in $M$ transverse to the flow of $X_{\mathcal{H}}$ which does not intersect any of its asymptotic limits.

Combining this with the above discussion, we see that if $\tilde{u}$ is furthermore connected, then $[\tilde{u}] *[\tilde{u}]=0$ implies that $\tilde{u}$ does not intersect any of its $\mathbb{R}$-translates and thus that the projected curve $u$ is an embedding (since it is an injective immersion that never intersects any of its asymptotic limits). We can therefore conclude that if the projected map $u$ is not an embedding then $[\tilde{u}] *[\tilde{u}]>0$. As with the discussion above concerning intersections of projections of curves to $M$, the converse is not true, since it could be the case that the generalized intersection number $[\tilde{u}] *\left[\tilde{u}_{c}\right]$ is positive, but still that the intersection number $\operatorname{int}\left(\tilde{u}, \tilde{u}_{c}\right)$ is zero for all $c \in \mathbb{R} \backslash\{0\}$.

However, again as above, the asymptotic intersection index of $\tilde{u}$ and $\tilde{u}_{c}$ changes in a predictable manner as $c$ varies and we can use this fact to come up with the following criteria for the map $u$ to be an embedding. As with Theorem 2.4, this result can be viewed as saying that certain subsets of the "obvious" necessary conditions for $u$ to be an embedding are also sufficient. In condition (4) below, the relative asymptotic intersection number

$$
i_{\infty}^{\Phi}([\tilde{u} ; z],[\tilde{u} ; w])
$$

is a count of how many intersections appear between a neighborhood of $z \in \Gamma$ and a neighborhood of $w \in \Gamma$ when $\tilde{u}$ is perturbed near $w$ in a direction determined by a trivialization $\Phi$ of the asymptotic limit of $\tilde{u}$ at $w$. More detail on this quantity can be found in Section 3.2 below.

Theorem 2.6 Let $[\Sigma, j, \Gamma, \tilde{u}=(a, u)] \in \mathcal{M}(M, \mathcal{H}, J)$ be a connected, simple pseudoholomorphic curve and assume that $\tilde{u}$ does not have image contained in an orbit cylinder. Then the following are equivalent: 
(1) The projected map $u: \Sigma \backslash \Gamma \rightarrow M$ is an embedding.

(2) The algebraic intersection number $\operatorname{int}\left(\tilde{u}, \tilde{u}_{c}\right)$ between $\tilde{u}$ and an $\mathbb{R}$-translate $\tilde{u}_{c}=(a+c, u)$ is zero for all $c \in \mathbb{R} \backslash\{0\}$.

(3) All of the following hold:

(a) $u$ does not intersect any of its asymptotic limits.

(b) If $\gamma$ is a periodic orbit so that $u$ is asymptotic at $z \in \Gamma$ to $\gamma^{m_{z}}$ and $u$ is asymptotic at $w \in \Gamma$ to $\gamma^{m_{w}}$, then

$$
\frac{\operatorname{wind}_{\infty}(\tilde{u} ; z)}{m_{z}}=\frac{\operatorname{wind}_{\infty}(\tilde{u} ; w)}{m_{w}} .
$$

(4) All of the following hold:

(a) The map $\tilde{u}$ is an embedding.

(b) The projected map $u$ is an immersion which is everywhere transverse to $X_{\mathcal{H}}$

(c) For each $z \in \Gamma$, we have

$$
\operatorname{gcd}\left(m_{z}, \operatorname{wind}_{\infty}(\tilde{u} ; z)\right)=1 .
$$

(d) If $\gamma$ is a periodic orbit so that $u$ is asymptotic at $z$ to $\gamma^{m_{z}}$ and $u$ is asymptotic at $w \neq z$ to $\gamma^{m_{w}}$ with $m_{z} m_{w}>0$, then the relative asymptotic intersection number of the ends $[\tilde{u} ; z]$ and $[\tilde{u} ; w]$ satisfies

$$
i_{\infty}^{\Phi}([\tilde{u} ; z],[\tilde{u} ; w])=-m_{z} m_{w} \max \left\{\frac{\operatorname{wind}_{\infty}^{\Phi}(\tilde{u} ; z)}{m_{z}}, \frac{\operatorname{wind}_{\infty}^{\Phi}(\tilde{u} ; w)}{m_{w}}\right\} .
$$

We note that the conditions of this theorem simplify somewhat if every asymptotic limit of $\tilde{u}$ is geometrically distinct. In particular, condition (3) implies that a simple curve with geometrically distinct asymptotic limits projects to an embedding in $M$ if and only if the projection does not intersect any of its asymptotic limits. For planes, this generalizes [8, Theorem 1.1].

As an application of some of these ideas, we can prove a result about the contact homology of a manifold admitting a holomorphic open book decomposition satisfying some additional assumptions. Recall that an open book decomposition of a 3-manifold $M$ is a pair $(L, \pi)$ where $L \subset M$ is a link and $\pi: M \backslash L \rightarrow S^{1}$ is a fibration, the fibers $\pi^{-1}(\theta)$ of which are embedded surfaces bounded by $L$. In this case the link $L$ is referred to as the binding of the open book decomposition and the fibers of the fibration are referred to as pages of the open book decomposition.

Now consider $M$ equipped with a stable Hamiltonian structure $\mathcal{H}$ and compatible complex multiplication $J \in \mathcal{J}(M, \mathcal{H})$. We say that $(M, \mathcal{H}, J)$ admits a holomorphic open book decomposition, if $M$ admits an open book decomposition $(L, \pi)$ so that the link $L$ is comprised of elliptic periodic orbits of the vector field $X_{\mathcal{H}}$ and the pages 
are projections of $\widetilde{J}$-holomorphic curves in $\mathbb{R} \times M$. We say a holomorphic open book decomposition is stable if the pseudoholomorphic curves which project to pages are genus 0 , have only positive simple punctures and have Fredholm index 2 .

Theorem 2.7 Assume that $(M, \mathcal{H}, J)$ admits a stable, holomorphic open book decomposition. Let $[\Sigma, j, \Gamma, \tilde{u}=(a, u)] \in \mathcal{M}(M, \mathcal{H}, J)$ be a connected pseudoholomorphic curve and assume that the image of $u$ is not a page of the open book decomposition and that $\tilde{u}$ does not have image contained in an orbit cylinder. Then at least one of the following is true:

(1) At least one of the positive punctures of $\tilde{u}$ limits to an orbit that is not a binding of the open book decomposition.

(2) At least one of the positive punctures of $\tilde{u}$ limits to a multiple cover of a binding orbit of the open book.

This result in particular immediately implies the following corollary. See Eliashberg, Givental and Hofer [4] for the definition of contact homology and cylindrical contact homology.

Corollary 2.8 Let $(M, \mathcal{H}, J)$ admit a stable, holomorphic open book decomposition $(L, \pi)$, let $\gamma \subset L$ be a binding orbit and assume that the contact homology (resp. cylindrical contact homology) of the triple $(M, \mathcal{H}, J)$ is well-defined. Then $\gamma$ is a cycle in the contact homology (resp. cylindrical contact homology) of $(M, \mathcal{H}, J)$.

2.4.2 Generalized pseudoholomorphic curves Finally we present a result concerning the intersection properties of so-called generalized pseudoholomorphic curves. These curves are introduced in Hofer [7] as a possible way to extend the theory of finite-energy foliations (see eg Hofer, Wysocki and Zehnder [12]) to include curves with nontrivial genus.

The generalized pseudoholomorphic curve equation for an asymptotically cylindrical map $(\Sigma, j, \Gamma,(a, u)) \in C^{\infty}(M, \mathcal{H})$ is obtained by twisting the first of the two equations in (2-6) by a harmonic form on $\Sigma$. More precisely, we consider quintuplets $(\Sigma, j, \Gamma, \tilde{u}=(a, u), v)$ where the quadruple $(\Sigma, j, \Gamma,(a, u))$ is a smooth asymptotically cylindrical map, (ie belongs to $C^{\infty}(M, \mathcal{H})$ ) and where $v$ is a 1 -form on the unpunctured surface $\Sigma$. Such a quintuple is called a generalized pseudoholomorphic map in $(M, \mathcal{H}, J)$ if it satisfies

$$
\left\{\begin{array}{c}
u^{*} \lambda \circ j=d a+v \\
\pi_{\xi_{\mathcal{H}} \circ d u \circ j}=J \circ \pi_{\xi^{\mathcal{H}}} \circ d u \\
d v=0=d(v \circ j) .
\end{array}\right.
$$


A generalized pseudoholomorphic curve is the equivalence class $[\Sigma, j, \Gamma, \tilde{u}=(a, u), v]$ of the quintuple $(\Sigma, j, \Gamma, \tilde{u}=(a, u), v)$ under the equivalence relation of holomorphic reparametrization of the domain.

As with pseudoholomorphic maps, a finite energy condition guarantees that the maps are asymptotically cylindrical. We will not give the definition of energy here, but will instead just assume that we are dealing with asymptotically cylindrical maps. We will denote the set of asymptotically cylindrical generalized pseudoholomorphic curves in $(M, \mathcal{H}, J)$ by $\mathcal{M}_{\Delta}(M, \mathcal{H}, J)$

In contrast to genuinely pseudoholomorphic curves, generalized pseudoholomorphic curves do not satisfy local positivity of intersections. Indeed, it is possible to construct a pair of local solutions $\tilde{u}=(a, u)$ and $\tilde{v}=(b, v)$ to (2-8) which have an isolated intersection of negative index (see Appendix B). In light of this, one might expect that a pair of generalized pseudoholomorphic curves could have arbitrary generalized intersection number, but as the following theorem shows, this is not the case.

Theorem 2.9 Let $[\Sigma, j, \Gamma, \tilde{u}, v],\left[\Sigma^{\prime}, j^{\prime}, \Gamma^{\prime}, \tilde{v}, v^{\prime}\right] \in \mathcal{M}_{\Delta}(M, \mathcal{H}, J)$ be generalized pseudoholomorphic curves and assume that no component of $\tilde{u}$ or $\tilde{v}$ is contained in an orbit cylinder. Then

$$
[\tilde{u}] *[\tilde{v}] \geq 0
$$

This result puts topological restrictions on what homotopy classes in $C^{\infty}(M, \mathcal{H})$ can contain generalized pseudoholomorphic maps. Indeed we have the following immediate corollaries of Theorem 2.9.

Corollary 2.10 Let $(\Sigma, j, \Gamma, \tilde{u}) \in C^{\infty}(M, \mathcal{H})$ be a smooth asymptotically cylindrical map with no component homotopic to an orbit cylinder. If

$$
[\tilde{u}] *[\tilde{u}]<0
$$

then there are no generalized pseudoholomorphic curves in the same relative homotopy class as $\tilde{u}$.

Corollary 2.11 Let $(\Sigma, j, \Gamma, \tilde{u}) \in C^{\infty}(M, \mathcal{H})$ be a smooth asymptotically cylindrical map with no component homotopic to an orbit cylinder. Assume there exists a generalized pseudoholomorphic curve $[\Sigma, j, \Gamma, \tilde{v}, v] \in \mathcal{M}_{\Delta}(M, \mathcal{H}, J)$ so that

$$
[\tilde{u}] *[\tilde{v}]<0 .
$$

Then there are no generalized pseudoholomorphic curves in the same relative homotopy class as $\tilde{u}$. 


\subsection{Overview}

The remainder of the paper is devoted to proving the results above concerning the properties and applications of generalized intersection number. We review relevant technical background material where appropriate.

As mentioned above, some difficulties arise in trying to understand the intersection and embedding properties of punctured pseudoholomorphic curves when multiple ends approach the same orbit or, when concerned with embedding controls, when an end approaches a multiple cover of an orbit. First, it is not a priori clear that the algebraic intersection number is finite since the domains are not compact. Second, even if the algebraic intersection number is finite, in families of curves, intersections (or selfintersections) can appear or disappear at the ends and thus, the algebraic intersection number is not topologically determined.

A precise understanding of the asymptotic behavior of punctured pseudoholomorphic curves is the key both to establishing finiteness of the algebraic intersection number and to understanding to what degree intersections or self-intersections can appear or disappear at the ends. The relevant asymptotic results are proved in [22] and are reviewed in Section 3.1.3 after reviewing facts about asymptotic operators and the Conley-Zehnder index in Section 3.1.1. With the appropriate asymptotic results in hand, we study some local asymptotic winding invariants in Section 3.1.4 which are convenient for encoding intersection related invariants we develop. Then in Section 3.2 we take up the study of local asymptotic intersection invariants. In particular we define and establish the main properties of the asymptotic intersection index $\delta_{\infty}([\tilde{u} ; z],[\tilde{v} ; w])$ of two ends and the asymptotic self-intersection index $\delta_{\infty}([\tilde{u} ; z])$ of a single end. For pseudoholomorphic curves, these quantities can, in light of the asymptotic results of [22], be thought of as measures respectively of tangency or self-tangency at infinity and, in this case, these quantities give the upper bound on the count of intersections or self-intersections that could appear that those ends.

The theorems stated above in Section 2.4 are then proved in Section 4 and Section 5 along with some complementary results. We establish the basic properties of the relative and generalized intersection numbers in Section 4.1 building up to the proof of Theorem 2.2. Section 4.2 is then devoted to proving Theorem 2.3 and some related results. The proofs of these theorems rest on the fact that, in the cases we consider, the generalized intersection number can be understood completely by combining facts about the local behavior of pseudoholomorphic curves from Micallef and White [18] with the asymptotic intersection invariants developed in Section 3. In Section 5, we then specialize to the case of a cylindrical cobordism. The key idea here is that the homotopy invariance of the generalized intersection number coupled with the existence 
of the $\mathbb{R}$-action allows the generalized intersection number to be computed in terms of quantities associated to the projection of the curve to the three-manifold.

Finally we close with two appendices. In Appendix A we adapt a proof of Hofer, Wysocki and Zehnder from [8] to show that the projection to $\xi^{\mathcal{H}}$ along $X_{\mathcal{H}}$ of the differential of a connected curve in $\mathcal{M}(M, \mathcal{H}, J)$ either vanishes identically or has a finite number of zeroes of positive order. In Appendix B we show that local solutions to the generalized pseudoholomorphic curve equations (2-8) can exhibit intersection behavior not found in genuinely pseudoholomorphic maps.

\section{Local intersection numbers at punctures}

\subsection{Asymptotics and asymptotic winding numbers}

3.1.1 Asymptotic operators and the Conley-Zehnder index Throughout this section, we will assume that $(M, \mathcal{H})$ is a 3 -manifold equipped with a stable Hamiltonian structure $\mathcal{H}=(\lambda, \omega)$ and that $J \in \mathcal{J}(M, \mathcal{H})$ is a compatible complex multiplication on $\xi^{\mathcal{H}}$.

We will associate to any periodic orbit a differential operator related to the linearized flow. Let $\gamma \in \mathcal{P}(M, \mathcal{H})$ be a $\tau$-periodic orbit (possibly multiply covered) and let $h$ be a vector field along $\gamma$, that is $h: S^{1} \rightarrow T M$ is a smooth function satisfying $h(t) \in T_{\gamma(t)} M$ for all $t \in S^{1}$. Since $h$ is defined along a flow line of $X_{\mathcal{H}}$ we can define the Lie derivative $L_{X_{\mathcal{H}}} h$ of $h$ by

$$
L_{X_{\mathcal{H}}} h(t)=\left.\frac{d}{d s}\right|_{s=0} d \psi_{-s}(\gamma(t+s / \tau)) h(t+s / \tau) .
$$

Since the flow $\psi_{t}$ of $X_{\mathcal{H}}$ preserves the splitting (2-1), so must $L_{X_{\mathcal{H}}}$ and we can conclude that if $h(t) \in \xi_{\gamma(t)}^{\mathcal{H}}$ for all $t \in S^{1}$, then $L_{X_{\mathcal{H}}} h(t) \in \xi_{\gamma(t)}^{\mathcal{H}}$ for all $t \in S^{1}$. Moreover, if $\nabla$ is a symmetric connection on $T M$, we can use $d \gamma(t) \partial_{t}=\tau \cdot X_{\mathcal{H}}(\gamma(t))$ to write

$$
\tau \cdot L_{X_{\mathcal{H}}} h=L_{\tau} \cdot X_{\mathcal{H}} h=\nabla_{\tau} \cdot X_{\mathcal{H}} h-\nabla_{h}\left(\tau \cdot X_{\mathcal{H}}\right)=\nabla_{t} h-\tau \nabla_{h} X_{\mathcal{H}},
$$

and therefore the differential operator $\nabla_{t} \cdot-\tau \nabla . X_{\mathcal{H}}$ maps sections of $\gamma^{*} \xi^{\mathcal{H}}$ to sections of $\gamma^{*} \xi^{\mathcal{H}}$ and is independent of the choice of symmetric connection.

Choosing some $J \in \mathcal{J}(M, \mathcal{H})$, we associate to each $\tau$-periodic orbit $\gamma \in \mathcal{P}_{\tau}(M, \mathcal{H})$ a differential operator $\mathbf{A}_{\gamma, J}: C^{\infty}\left(\gamma^{*} \xi^{\mathcal{H}}\right) \rightarrow C^{\infty}\left(\gamma^{*} \xi^{\mathcal{H}}\right)$ acting on the space of smooth sections of $\xi^{\mathcal{H}}$ along $\gamma$ defined by

$$
\mathbf{A}_{\gamma, J} \eta=-J\left(\nabla_{t} \eta-\tau \nabla_{\eta} X_{\mathcal{H}}\right)
$$


We note that the discussion of the previous paragraph implies that $\mathbf{A}_{\gamma, J}$ does in fact map the space of sections of $\xi^{\mathcal{H}}$ along $\gamma$ to itself and that $\mathbf{A}_{\gamma, J}$ is independent of symmetric connection $\nabla$ used to define it. Define an inner product on $C^{\infty}\left(\gamma^{*} \xi^{\mathcal{H}}\right)$ by

$$
\langle h, k\rangle_{J}=\int_{S^{1}} \omega_{\gamma(t)}(h(t), J(\gamma(t)) k(t)) d t .
$$

Recalling that $L_{X_{\mathcal{H}}} \omega=0$, we have for any $h, k \in C^{\infty}\left(\gamma^{*} \xi^{\mathcal{H}}\right)$ that

$$
\frac{\partial}{\partial t} \omega_{\gamma(t)}(h(t), k(t))=\omega_{\gamma(t)}\left(\tau\left(L_{X_{\mathcal{H}}} h\right)(t), k(t)\right)+\omega_{\gamma(t)}\left(h(t), \tau\left(L_{X_{\mathcal{H}}} k\right)(t)\right)
$$

Noting that the compatibility of $J$ with $\left.\omega\right|_{\xi \mathcal{H}}$ implies that $\omega(J \cdot, J \cdot)=\omega(\cdot, \cdot)$ on $\xi^{\mathcal{H}} \times \xi^{\mathcal{H}}$, we integrate this to give

$$
\left\langle h, \mathbf{A}_{\gamma, J} k\right\rangle_{J}=\left\langle\mathbf{A}_{\gamma, J} h, k\right\rangle_{J}
$$

Therefore $\mathbf{A}_{\gamma, J}$ is formally self-adjoint and $\mathbf{A}_{\gamma, J}$ induces a self-adjoint operator

$$
\mathbf{A}_{\gamma, J}: D\left(\mathbf{A}_{\gamma, J}\right)=H^{1}\left(\gamma^{*} \xi^{\mathcal{H}}\right) \subset L^{2}\left(\gamma^{*} \xi^{\mathcal{H}}\right) \rightarrow L^{2}\left(\gamma^{*} \xi^{\mathcal{H}}\right) .
$$

We will refer to $\mathbf{A}_{\gamma, J}$ as the asymptotic operator associated to the orbit $\gamma$.

The kernel of $\mathbf{A}_{\gamma, J}$ is closely related to the degeneracy of the orbit $\gamma$. Indeed, if $\gamma$ is degenerate then there is a nonzero $v_{0} \in \xi_{\gamma(0)}^{\mathcal{H}}$ so that $d \psi_{\tau}(\gamma(0)) v_{0}=v_{0}$. Defining $v \in C^{\infty}\left(\gamma^{*} \xi^{\mathcal{H}}\right)$ by $v(t)=d \psi_{\tau t}(\gamma(0)) v_{0}$, we have that $L_{X_{\mathcal{H}}} v \equiv 0$, so $v \in \operatorname{ker} \mathbf{A}_{\gamma, J}$. Conversely, if $h \in C^{\infty}\left(\gamma^{*} \xi^{\mathcal{H}}\right)$ satisfies $h \in \operatorname{ker} \mathbf{A}_{\gamma, J}$, then we can conclude that $L_{X_{\mathcal{H}}} h \equiv 0$ so we must have that $h(t)=d \psi_{\tau t}(\gamma(0)) h(0)$ and in particular $h(0)=$ $d \psi_{\tau}(\gamma(0)) h(0)$. Therefore $\gamma$ is nondegenerate if and only if $\mathbf{A}_{\gamma, J}$ has trivial kernel.

It will be important to understand the behavior - particularly the winding - of eigenvectors of asymptotic operators associated to periodic orbits in trivializations of $\xi^{\mathcal{H}}$. We establish our conventions for dealing with trivializations here. Given a simple periodic orbit $\gamma \in \mathcal{P}^{0}(M, \mathcal{H})$ we will use the term unitary trivialization of $\left(\gamma^{*} \xi^{\mathcal{H}}, \omega, J\right)$ to refer to a trivialization

$$
\Phi_{\gamma}: S^{1} \times\left.\mathbb{R}^{2} \rightarrow \xi^{\mathcal{H}}\right|_{\gamma}
$$

of $\gamma^{*} \xi^{\mathcal{H}}$ satisfying

$$
\begin{gathered}
\omega\left(\Phi_{\gamma}, \Phi_{\gamma} \cdot\right)=d x \wedge d y \\
\Phi_{\gamma} \circ J_{0}=J \circ \Phi_{\gamma}
\end{gathered}
$$

where $J_{0}$ is the standard complex multiplication used to identify $\mathbb{R}^{2}$ with $\mathbb{C}$. Having chosen a unitary trivialization $\Phi_{\gamma}$ of $\left(\gamma^{*} \xi^{\mathcal{H}}, \omega, J\right)$, we get an induced trivialization for $\xi^{\mathcal{H}}$ along the multiply covered orbit $\left(\left(\gamma^{m}\right)^{*} \xi^{\mathcal{H}}, \omega, J\right)$ by pulling back $\Phi_{\gamma}$ via the

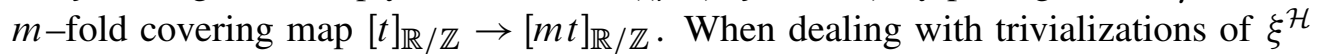


along an orbit $\gamma^{m}$, we will always assume that the trivialization arises from a choice of trivialization along the underlying simply covered orbit $\gamma \in \widetilde{\mathcal{P}}^{0}(M, \mathcal{H})$. Moreover, we will generally use the same notation to indicate the trivialization $\Phi$ of $\xi^{\mathcal{H}}$ along $\gamma$ and that induced on $\xi^{\mathcal{H}}$ along $\gamma^{m}$.

In a unitary trivialization of the $\left(\gamma^{*} \xi^{\mathcal{H}}, \omega, J\right)$ along a simple periodic orbit $\gamma: S^{1} \rightarrow M$, the asymptotic operator $\mathbf{A}_{\gamma^{m}, J}$ takes the form

$$
\left(\mathbf{A}_{\gamma^{m}, J} h\right)(t)=-i \frac{d}{d t} h(t)-S(t) h(t),
$$

where $S(t)$ is a symmetric, two-by-two matrix. An eigenvector of $\mathbf{A}_{\gamma^{m}, J}$ satisfies a linear, first-order ordinary differential equation and therefore never vanishes since it does not vanish identically. Hence every eigenvector gives a map from $S^{1} \rightarrow \mathbb{R}^{2} \backslash\{0\}$ and thus has a well defined winding number. Since $-i \frac{d}{d t}-S(t)$ is a compact perturbation of $-i \frac{d}{d t}$, it can be shown that the winding is monotonic in the eigenvalue and that to any $k \in \mathbb{Z}$ there is a two dimensional space of eigenvectors with winding $k$. These results are proved in $[8$, Section 3$]$ and we restate them here as a lemma.

Lemma 3.1 Let $\gamma \in \mathcal{P}^{0}(M, \mathcal{H})$ be a simple periodic orbit of $X_{\mathcal{H}}$, let $\mathbf{A}_{\gamma^{m}, J}$ denote the asymptotic operator of $\gamma^{m}$ for $m \in \mathbb{Z} \backslash\{0\}$ and let $\mathfrak{T}\left(\gamma^{*} \xi^{\mathcal{H}}\right)$ denote the set of homotopy classes of unitary trivializations of $\left(\gamma^{*} \xi^{\mathcal{H}}, \omega, J\right)$. There exists a map $w: \sigma\left(\mathbf{A}_{\gamma^{m}, J}\right) \times \mathfrak{T}\left(\gamma^{*} \xi^{\mathcal{H}}\right) \rightarrow \mathbb{Z}$ which satisfies

(1) If $e: S^{1} \rightarrow\left(\gamma^{m}\right)^{*} \xi^{\mathcal{H}}$ is an eigenvector of $\mathbf{A}_{\gamma^{m}, J}$ with eigenvalue $\lambda$, then $w(\lambda,[\Phi])=\operatorname{wind}\left(\Phi^{-1} e\right)$, that is, $w(\lambda,[\Phi])$ measures the winding with respect to $\Phi$ of any eigenvector of $\mathbf{A}_{\gamma^{m}}, J$ with eigenvalue $\lambda$.

(2) For any fixed $[\Phi] \in \mathfrak{T}\left(\gamma^{*} \xi^{\mathcal{H}}\right)$ we have that

$$
w(\lambda,[\Phi])<w(\mu,[\Phi]) \Rightarrow \lambda<\mu,
$$

that is, the winding of eigenvectors of $\mathbf{A}_{\gamma^{m}, J}$ is (not strictly) monotonic in the eigenvalue.

(3) If $m(\lambda)=\operatorname{dim} \operatorname{ker}\left(\mathbf{A}_{\gamma^{m}}, J-\lambda\right)$ denotes the multiplicity of $\lambda$ as an eigenvalue we have for every $k \in \mathbb{Z}$ and $[\Phi] \in \mathfrak{T}\left(\gamma^{*} \xi^{\mathcal{H}}\right)$ that

$$
\sum_{\{\lambda \mid w(\lambda,[\Phi])=k\}} m(\lambda)=2,
$$

that is, the space of eigenvectors of $\mathbf{A}_{\gamma^{m}, J}$ with any given winding has dimension 2 . 
In this paper the relationship between the spectrum of the asymptotic operator associated to a simply covered orbit $\gamma$ and that of the asymptotic operator associated to its $k$-fold iterate $\gamma^{k}$ will be important. First note that there is a $\mathbb{Z}_{k}$ action on sections of $\left(\gamma^{k}\right)^{*} \xi^{\mathcal{H}}$

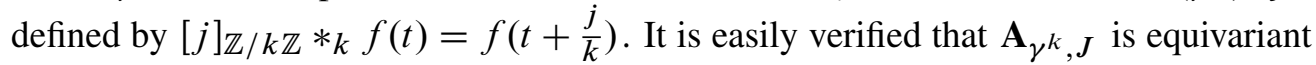
with respect to this action and therefore that the eigenspaces of $\mathbf{A}_{\gamma^{k}, J}$ are fixed by this action. We say an eigenvector $e$ of $\mathbf{A}_{\gamma^{k}, J}$ is multiply covered if the isotropy group

$$
G(e)=\left\{j \in \mathbb{Z}_{k} \mid j *_{k} e=e\right\}
$$

is nontrivial and we define the covering number of $e$

$$
\operatorname{cov}(e)=|G(e)|
$$

of $e$ to be the order of this group. Put more $\operatorname{simply}, \operatorname{cov}(e)$ is the largest positive integer $m$ dividing $k$ for which $(k / m) *_{k}$. fixes $e$. It follows from the definition of $\mathbf{A}_{\gamma, J}$ that if $e$ is an eigenvector of $\mathbf{A}_{\gamma, J}$ with eigenvalue $\lambda$, then the section $e_{k} \in C^{\infty}\left(\left(\gamma^{k}\right)^{*} \xi^{\mathcal{H}}\right)$ defined by $e_{k}(t)=e(k t)$ is an eigenvector of $\mathbf{A}_{\gamma^{k}, J}$ with eigenvalue $k \lambda$. Furthermore, it is straightforward to see that any eigenvector $e$ of $\mathbf{A}_{\gamma^{k}, J}$ with covering number $m=\operatorname{cov}(e)$ is of the form $e(t)=f(m t)$ for some eigenvector $f$ of $\mathbf{A}_{\gamma^{k / m}, J}$ with $\operatorname{cov}(f)=1$.

The following lemma is an easy consequence of the preceding discussion and Lemma 3.1.

Lemma 3.2 If $e$ is an eigenvector of $\mathbf{A}_{\gamma^{k}, J}$ and $\Phi$ is a trivialization of $\gamma^{*} \xi^{\mathcal{H}}$, then

$$
\operatorname{cov}(e)=\operatorname{gcd}\left(\operatorname{wind}\left(\Phi^{-1} e\right), k\right) .
$$

Following [8] we define the Conley-Zehnder index of a periodic orbit $\gamma^{k}$ in terms of the spectrum of the asymptotic operator $\mathbf{A}_{\gamma^{k}, J}$. We define a number $\sigma_{\text {max }}^{-}\left(\gamma^{k}\right) \in \sigma\left(\mathbf{A}_{\gamma^{k}, J}\right)$ by

$$
\sigma_{\max }^{-}\left(\gamma^{k}\right)=\max \left(\sigma\left(\mathbf{A}_{\gamma^{k}, J}\right) \cap \mathbb{R}^{-}\right)
$$

ie so that $\sigma_{\text {max }}^{-}\left(\gamma^{k}\right)$ is the largest negative eigenvalue of $\mathbf{A}_{\gamma^{k}, J}$. Given a trivialization $\Phi$ of $\gamma^{*} \xi^{\mathcal{H}}$, we define

$$
\left.\alpha^{\Phi}\left(\gamma^{k}\right)=w\left(\sigma_{\max }^{-}\left(\gamma^{k}\right) ; \Phi\right]\right)
$$

so that $\alpha^{\Phi}\left(\gamma^{k}\right)$ is the winding relative to $\Phi$ of any eigenvector of $\mathbf{A}_{\gamma^{k}, J}$ having the largest possible negative eigenvalue. We define the parity of $p\left(\gamma^{k}\right)$ of $\gamma^{k}$ by

$$
p\left(\gamma^{k}\right)= \begin{cases}0 & \text { if } \exists \mu \in \sigma\left(\mathbf{A}_{\gamma^{k}, J}\right) \cap \mathbb{R}^{+} \text {with } w(\mu,[\Phi])=\alpha^{\Phi}\left(\gamma^{k}\right) \\ 1 & \text { otherwise }\end{cases}
$$


and we note that this definition does not depend on the choice of $\Phi$. We recall from [8] that the parity defined here agrees with the designation of $\gamma^{k}$ as even or odd as defined in Section 2.1. The Conley-Zehnder index $\mu^{\Phi}\left(\gamma^{k}\right)$ of the orbit $\gamma^{k}$ relative to the trivialization $\Phi$ is then defined by

$$
\mu^{\Phi}\left(\gamma^{k}\right)=2 \alpha^{\Phi}\left(\gamma^{k}\right)+p\left(\gamma^{k}\right)
$$

We will at times suppress the choice of trivialization in our notation for $\alpha$ or $\mu$, but it should always be understood that a choice of trivialization is necessary to define these quantities. We also observe that even though the asymptotic operator $\mathbf{A}_{\gamma^{k}, J}$ depends on a choice of $J \in \mathcal{J}(M, \mathcal{H})$, the Conley-Zehnder index of an orbit is independent of this choice, as can be seen by the alternate definitions given in [8].

Understanding how the Conley-Zehnder index of $\gamma^{k}$ behaves as a function of $k$ for fixed $\gamma$ will be important. This is well understood and the important facts will be listed in the following lemma, the proof of which follows from basic facts about the symplectic group $S p(1)$ which can be found for example in [12, Appendix 8.1] or the first chapter of Abbondandolo [1]. For our purposes, it will be more convenient to state an iteration formula for $\alpha$ rather than $\mu$, but the relationship between these two is clear.

Lemma 3.3 Let $\gamma$ be a (not necessarily simple) periodic orbit and assume that $\gamma^{m}$ is nondegenerate for all $m \in \mathbb{Z} \backslash\{0\}$. Let $k \in \mathbb{Z} \backslash\{0\}$ be a nonzero integer.

- If $\gamma$ is an even orbit, then

$$
\alpha\left(\gamma^{k}\right)=k \alpha(\gamma)
$$

- If $\gamma$ is an odd hyperbolic orbit, then

$$
\alpha\left(\gamma^{k}\right)=\left\lfloor k\left(\alpha(\gamma)+\frac{1}{2}\right)\right\rfloor=k \alpha(\gamma)+\frac{k-p(k)}{2}
$$

where $\lfloor\cdot\rfloor$ is the least integer function and $p(k)$ denotes the parity of the integer $k$.

- If $\gamma$ is an elliptic orbit, then there exists an irrational $\theta \in \mathbb{R}$ so that

$$
\alpha\left(\gamma^{k}\right)=\lfloor k \theta\rfloor \text {. }
$$

It will be convenient for later to record how $\alpha$ changes with changes of trivialization. The only subtlety here is that since we always deal with trivializations of the underlying simply covered orbits, we get a factor which accounts for the covering number of the orbit. We note that if $E \rightarrow S^{1}$ is a Hermitian line bundle with unitary trivializations $\Phi$ and $\Psi$, the map $\Phi^{-1} \circ \Psi$ determines a map $S^{1} \rightarrow U(1) \approx S^{1}$. We denote the degree 
of this map by $\operatorname{deg}\left(\Phi^{-1} \circ \Psi\right)$. We now state the formula as a lemma and omit the easy proof.

Lemma 3.4 Let $\Phi$ and $\Psi$ be trivializations of $\gamma^{*} \xi^{\mathcal{H}}$. Then

$$
\alpha^{\Phi}\left(\gamma^{k}\right)=\alpha^{\Psi}\left(\gamma^{k}\right)+k \operatorname{deg}\left(\Phi^{-1} \circ \Psi\right)
$$

for any nonzero $k \in \mathbb{Z}$.

We close this section by introducing a quantity that will be useful later. Given any eigenvalue $\mu \in \sigma\left(\mathbf{A}_{\gamma^{k}, J}\right)$ it is clear from Lemma 3.1 and Lemma 3.2 that all eigenvectors with eigenvalue $\mu$ have the same covering number. We will therefore write

$$
\operatorname{cov}(\mu)
$$

to denote the covering number of any eigenvector with eigenvalue $\mu$. Given any simple periodic orbit $\gamma \in \mathcal{P}^{0}(M, \mathcal{H})$, we will then define the spectral covering number $\bar{\sigma}\left(\gamma^{k}\right)$ of $\gamma^{k}$ to be

$$
\bar{\sigma}\left(\gamma^{k}\right):=\operatorname{cov}\left(\sigma_{\max }^{-}\left(\gamma^{k}\right)\right)
$$

and note that by Lemma 3.2 that

$$
\bar{\sigma}\left(\gamma^{k}\right)=\operatorname{gcd}\left(k, \alpha^{\Phi}\left(\gamma^{k}\right)\right)
$$

where $\alpha^{\Phi}\left(\gamma^{k}\right)$ is computed relative to any trivialization of $\gamma^{*} \xi^{\mathcal{H}}$. Note that even though $\alpha^{\Phi}\left(\gamma^{k}\right)$ depends on a choice of trivialization, Lemma 3.4 above shows that changing the trivialization changes $\alpha$ by an integer multiple of $k$, so the gcd on the right hand side of this equation is unchanged.

We note that as a consequence of Lemma 3.3, the spectral covering number of a hyperbolic orbit can be computed knowing just the covering number of the orbit and whether the underlying simple orbit is even or odd. We state this result as a lemma and omit the straightforward proof.

Lemma 3.5 Let $\gamma$ be simple periodic orbit and let $k \in \mathbb{Z} \backslash\{0\}$.

- If $\gamma$ is an even orbit, then

$$
\bar{\sigma}\left(\gamma^{k}\right)=|k| .
$$

- If $\gamma$ is an odd, hyperbolic orbit, then

$$
\bar{\sigma}\left(\gamma^{k}\right)= \begin{cases}|k| / 2 & \text { if } k \text { is even } \\ 1 & \text { if } k \text { is odd. }\end{cases}
$$


3.1.2 Asymptotically cylindrical ends Here we will introduce a notion that will be useful for framing the results of the next several sections. In this section we continue to consider a 3-manifold $M$ equipped with a stable Hamiltonian structure $\mathcal{H}=(\lambda, \omega)$ and a compatible $J \in \mathcal{J}(M, \mathcal{H})$.

Consider quadruples of the form $\left(\Sigma, j, z_{*}, u\right)$ where $(\Sigma, j)$ is a (not necessarily closed) Riemann surface without boundary, $z_{*} \in \Sigma$ is a point and $u: \Sigma \backslash\left\{z_{*}\right\} \rightarrow \mathbb{R} \times M$ is a smooth map. We will call such a quadruple an asymptotically cylindrical end model if there is a periodic orbit $\gamma^{m}$ so that $u$ is asymptotically cylindrical over $\gamma^{m}$.

We define an equivalence relation on end models in the following way. We say that asymptotically cylindrical end models $\left(\Sigma, j, z_{*}, u\right)$ and $\left(\Sigma^{\prime}, j^{\prime}, w_{*}, v\right)$ are equivalent if there exists an open neighborhood $U \subset \Sigma$ of $z_{*}$ and a holomorphic embedding $\psi: U \rightarrow \Sigma^{\prime}$ with $\psi\left(z_{*}\right)=w_{*}$ so that

$$
u=v \circ \psi \text {. }
$$

on $U \backslash\left\{z_{*}\right\}$. An equivalence class $\left[\Sigma, j, z_{*}, u\right]$ of asymptotically cylindrical end models will be referred to as an asymptotically cylindrical end.

An asymptotically cylindrical end is said to be embedded if it has a representative model $\left(\Sigma, j, z_{*}, u\right)$ for which $u$ is an embedding. A pair of asymptotically cylindrical ends are said to be nonintersecting, if they can be represented by models $\left(\Sigma, j, z_{*}, u\right)$ and $\left(\Sigma^{\prime}, j^{\prime}, w_{*}, v\right)$ satisfying

$$
u\left(\Sigma \backslash\left\{z_{*}\right\}\right) \cap v\left(\Sigma^{\prime} \backslash\left\{w_{*}\right\}\right)=\varnothing .
$$

An asymptotically cylindrical end is said to be pseudoholomorphic if it can be represented by a model $\left(\Sigma, j, z_{*}, u\right)$ with $u:\left(\Sigma \backslash\left\{z_{*}\right\}, j\right) \rightarrow(\mathbb{R} \times M, \widetilde{J})$ a pseudoholomorphic map.

By choosing holomorphic coordinates near a given point, it is clear that any asymptotically cylindrical end can be represented by a model of the form $(\mathbb{D}, i, 0, u)$, where $\mathbb{D}$ is the unit disk in $\mathbb{C}$ centered at $0 \in \mathbb{C}$. We will call such a model, a unit disk model. Given an asymptotically cylindrical end $[\mathbb{D}, i, 0, u]$ and a positive integer $m$, we can define the $m$-multiple cover $m \cdot[\mathbb{D}, i, 0, u]$ of $[\mathbb{D}, i, 0, u]$ by

$$
m \cdot[\mathbb{D}, i, 0, u]:=\left[\mathbb{D}, i, 0, u \circ \phi_{m}\right]
$$

where $\phi_{m}: \mathbb{D} \rightarrow \mathbb{D}$ is the map $\phi(z)=z^{m}$. It is a straightforward exercise in complex analysis to verify that the equivalence class of the end-model produced in this way does not depend on the choice of unit disk model used in the definition.

It will be convenient for some of the following results to consider asymptotically cylindrical ends with one additional piece of data. We will call a quintuple $\left(\Sigma, j, z_{*}, \bar{v}, u\right)$ 
a decorated asymptotically cylindrical end model if the quadruple $\left(\Sigma, j, z_{*}, u\right)$ is an asymptotically cylindrical end model and

$$
\bar{v} \in \Sigma_{z_{*}}^{0}:=\left(T_{z_{*}} \Sigma \backslash\{0\}\right) / \mathbb{R}^{+}
$$

is a ray in the tangent space of $\Sigma$ at $z_{*}$. The ray in the data for a decorated end model will be referred to as an asymptotic marker or decoration of the end. Two decorated end models $\left(\Sigma, j, z_{*},[X]_{\Sigma_{z *}^{0}}, u\right)$ and $\left(\Sigma^{\prime}, j^{\prime}, w_{*},[Y]_{\Sigma_{\Sigma_{*}^{\prime}}^{\prime 0}}, v\right)$ are said to be equivalent when there is an open neighborhood $U \subset \Sigma$ of $z_{*}$ and a holomorphic embedding $\psi: U \rightarrow \Sigma^{\prime}$ with $\psi\left(z_{*}\right)=w_{*},\left[d \psi\left(z_{*}\right) X\right]_{\Sigma^{\prime}{ }_{w *}^{0}}=[Y]_{\Sigma^{\prime}{ }_{w *}^{0}}$ and

$$
u=v \circ \psi
$$

on $U \backslash\left\{z_{*}\right\}$. An equivalence class of decorated end models is called a decorated asymptotically cylindrical end.

It is clear that any decorated asymptotically cylindrical end can be represented by a model of the form $\left(\mathbb{D}, i, 0,[1]_{\mathbb{C}^{*} / \mathbb{R}^{+}}, u\right)$. As with undecorated ends, we can define the $m$-multiple cover by

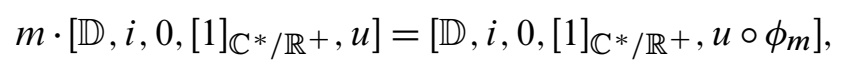

and it is easily verified that the equivalence class of end models produced in this way does not depend on the unit disk model chosen to represent the end.

Finally, given a decorated end $\left[\Sigma, j, z_{*},[X]_{\Sigma_{z *}^{0}}, u\right]$ and $a \in \mathbb{C}^{*}$ we define the change of decoration map taking $\left[\Sigma, j, z_{*},[X]_{\Sigma_{z *}^{0}}, u\right]$ to $a *\left[\Sigma, j, z_{*},[X]_{\Sigma_{z *}^{0}}, u\right]$ by defining

$$
a *\left[\Sigma, j, z_{*},[X]_{\Sigma_{z *}^{0}}, u\right]=\left[\Sigma, j, z_{*},[a X]_{\Sigma_{z *}^{0}}, u\right]
$$

and again, it is easily verified that this is a well-defined operation on ends independent of the choice of representing model and $X \in[X]_{\Sigma_{* *}^{0}}$.

3.1.3 Asymptotic normal forms and some consequences Underlying all of the results of this paper is a precise description of the asymptotic behavior of pseudoholomorphic half-cylinders and of the relative asymptotic behavior of a pair of half-cylinders limiting to the same periodic orbit. The key results are proved in [22] which builds on previous results from Hofer, Wysocki and Zehnder [10; 9], Kriener [16] and MoraDonato [20]. In this section, we will review the relevant facts and some consequences. Here we continue to assume $(M, \mathcal{H}, J)$ to be a 3 -manifold equipped with a stable Hamiltonian structure and compatible complex multiplication, but we now assume $\mathcal{H}$ to be nondegenerate. 
Let $\left[\Sigma, j, z_{*},[X], \tilde{u}=(a, u)\right]$ be a decorated asymptotically cylindrical end in $(\mathbb{R} \times$ $M, \widetilde{J})$ and assume that $u$ is asymptotically cylindrical over $\gamma^{k}$ for some $\tau$-periodic $\gamma \in \widetilde{\mathcal{P}}^{0}(M, \mathcal{H})$ and $k \in \mathbb{Z} \backslash\{0\}$. Moreover, we assume that if $\sigma:[0, \varepsilon) \rightarrow \Sigma$ is a smooth curve satisfying $\sigma(0)=z_{*}$ and $\sigma^{\prime}(0) \in[X]$, then $\lim _{t \rightarrow 0} u(\sigma(t))=\gamma(0)$, ie if we extend the projected map $u$ to the $S^{1}$ compactification of $\Sigma \backslash\left\{z_{*}\right\}$, the asymptotic marker $[X]$ hits the point $\gamma(0)$ on the orbit.

The assumption that $\tilde{u}$ is asymptotically cylindrical allows us to find for some $R \in \mathbb{R}$ an embedding $^{2} \psi:[R, \infty) \times S^{1} \rightarrow \Sigma \backslash \Gamma$, with

$$
\begin{gathered}
\lim _{s \rightarrow \infty} \psi(s, t)=z_{*} \\
\lim _{s \rightarrow \infty}-\frac{\partial_{s} \psi(s, 0)}{\left|\partial_{s} \psi(s, 0)\right|} \in[X] \in\left(T_{z_{*}} \Sigma \backslash\{0\}\right) / \mathbb{R}^{+}
\end{gathered}
$$

and a map $U:[R, \infty) \times S^{1} \rightarrow\left(\gamma^{k}\right)^{*} \xi^{\mathcal{H}}$ with $U(s, t) \in \xi_{\gamma^{k}(t)}^{\mathcal{H}}$ for all $(s, t) \in[R, \infty) \times S^{1}$ so that

$$
\tilde{u}(\psi(s, t))=\left(k \tau s, \exp _{\gamma^{k}(t)} U(s, t)\right),
$$

where exp denotes the exponential map of the metric

$$
g_{\mathcal{H}, J}:=\lambda \otimes \lambda+\omega(\cdot, J \cdot)
$$

on $M$. We will call a pair (U, $\psi$ ) satisfying (3-10)-(3-11) an asymptotic representative of $\left[\Sigma, j, z_{*},[X], u\right]$. It is clear from the $C^{1}$ convergence of $\tilde{u}$ to $\mathbb{R} \times \gamma^{k}$ that (3-10) and (3-11) uniquely determine $(U, \psi)$ up to restriction of the domain.

Now let $\left[\Sigma^{\prime}, j^{\prime}, w_{*},[Y], \tilde{v}\right]$ be a second decorated asymptotically cylindrical end, which is also asymptotically cylindrical over $\gamma^{k}$. Then we can find an asymptotic representative $(V, \phi)$ of $\tilde{v}$ near $w_{*}$ so that we can write

$$
\tilde{v}(\phi(s, t))=\left(k \tau s, \exp _{\gamma^{k}(t)} V(s, t)\right)
$$

If the ends being considered are pseudoholomorphic, the asymptotic behavior of the difference of the maps $U$ and $V$ is given by the following theorem. The proof can be found in [22].

${ }^{2}$ We remark that the embedding $\psi$ above is not in general holomorphic with respect to the standard almost complex structure $j_{0}$ on $[R, \infty) \times S^{1} \subset \mathbb{R} \times S^{1}=\mathbb{C} / i \mathbb{Z}$ even if the end is pseudoholomorphic. However it is easily seen from the results in [22] and the fact that nondegeneracy implies exponential convergence that if the end is pseudoholomorphic, then there exist positive constants $d$ and $M$ so that

$$
\left|\bar{\partial}_{j, j_{0}} \psi(s, t)\right|=\left|\frac{1}{2}\left(d \psi+j \circ d \psi \circ j_{0}\right)(s, t)\right| \leq M e^{-(d+2 \pi) s}
$$

for all $(s, t) \in[R, \infty) \times S^{1}$, where the norm $|\cdot|$ on $\operatorname{Hom}^{0,1}\left(\mathbb{R} \times S^{1}, T \Sigma\right)$ is defined with respect to the euclidean metric on $\mathbb{R} \times \mathbb{R} / \mathbb{Z}$ and any metric on the unpunctured surface $\Sigma$. 
Theorem 3.6 Assume that $\left[\Sigma, j, z_{*},[X], \tilde{u}\right]$ and $\left[\Sigma^{\prime}, j^{\prime}, w_{*},[Y], \tilde{v}\right]$ are distinct, decorated pseudoholomorphic ends asymptotically cylindrical over $\gamma^{k}$, with asymptotic representatives $(U, \psi)$ and $(V, \phi)$ respectively. Then the difference $U-V$, where defined, can be written

$$
U(s, t)-V(s, t)=e^{\lambda s}[e(t)+r(s, t)]
$$

where $e$ is an eigenvector of the asymptotic operator $\mathbf{A}_{\gamma^{k}, J}$ with eigenvalue $\lambda<0$ and where $r$ satisfies

$$
\left|\nabla_{s}^{i} \nabla_{t}^{j} r(s, t)\right| \leq M_{i j} e^{-d s}
$$

for all $(s, t) \in[R, \infty) \times S^{1},(i, j) \in \mathbb{N}^{2}$ and some appropriate constants $d>0$, $M_{i j}>0$.

Some remarks about this theorem are in order. We first remark that if $(U, \phi)$ is an asymptotic representative of $\left[\Sigma, j, z_{*},[X], \tilde{u}\right]$, then $\left(U\left(\cdot, \cdot+\frac{1}{k}\right), \phi\left(\cdot, \cdot+\frac{1}{k}\right)\right)$ is an asymptotic representative of the end

$$
e^{i 2 \pi / k} *\left[\Sigma, j, z_{*},[X], \tilde{u}\right]=\left[\Sigma, j, z_{*},\left[e^{i 2 \pi / k} X\right], \tilde{u}\right] .
$$

Thus if $e^{i 2 \pi l / k} *\left[\Sigma, j, z_{*},[X], \tilde{u}\right] \neq e^{i 2 \pi j / k} *\left[\Sigma^{\prime}, j^{\prime}, w_{*},[Y], \tilde{v}\right]$, the previous theorem then lets us write

$$
U\left(s, t+\frac{l}{k}\right)-V\left(s, t+\frac{j}{k}\right)=e^{\lambda_{l j}}\left[e_{l j}(t)+r_{l j}(s, t)\right]
$$

with each $\lambda_{l j}, e_{l j}$ and $r_{l j}$ satisfying that same properties as the $\lambda, e$ and $r$ appearing in the theorem. When $|k|>1$, we can apply this observation to the case where $\tilde{v}=\tilde{u}$ so the theorem gives an asymptotic description of each of the maps $U\left(s, t+\frac{i}{k}\right)-U\left(s, t+\frac{j}{k}\right)$. Additionally, we remark that in the case that $\tilde{v}$ is a cylinder over $\gamma^{k}$, we get that $V \equiv 0$, so this theorem reduces to give an asymptotic description of a single half-cylinder as in $[10 ; 20]$. Combining these observations, we get the following description of the asymptotic behavior of $U$ which is a refinement of the results of $[10 ; 20]$ in the event that $|k|>1$. For proof, see [22].

Theorem 3.7 With $(U, \phi)$ as defined above, there exists an $s_{0} \in \mathbb{R}$ so that for $(s, t) \in$ $\left[s_{0}, \infty\right) \times S^{1}$, either $U$ vanishes identically or

$$
U(s, t)=\sum_{i=1}^{N} e^{\lambda_{i} s}\left(e_{i}(t)+r_{i}(s, t)\right)
$$

where

- The $\lambda_{i}$ are a sequence of negative eigenvalues of $\mathbf{A}_{\gamma^{k}, J}$ which is strictly decreasing in $i$ (ie $\lambda_{j}<\lambda_{i}$ for $j>i$ ). 
- Each $e_{i}(\neq 0)$ is an eigenvector of $\mathbf{A}_{\gamma^{k}, J}$ with eigenvalue $\lambda_{i}$.

- The sequence of positive integers defined by setting $k_{1}=\operatorname{cov}\left(e_{1}\right)$ and $k_{i}=$ $\operatorname{gcd}\left(k_{i-1}, \operatorname{cov}\left(e_{i}\right)\right)$, is strictly decreasing in $i$.

- The $r_{i}$ satisfy $r_{i}(s, t)=r_{i}\left(s, t+\frac{1}{k_{i}}\right)$. Moreover, each $r_{i}$ satisfies exponential decay estimates of the form

$$
\left|\nabla_{s}^{l} \nabla_{t}^{m} r_{i}(s, t)\right|<M_{l m} e^{-d s}
$$

The following two corollaries will be important for our later results. For proofs see [22].

Corollary 3.8 Let $\left[\Sigma, j, z_{*}, \tilde{u}\right]$ and $\left[\Sigma^{\prime}, j^{\prime}, w_{*}, \tilde{v}\right]$ be pseudoholomorphic ends in $\mathbb{R} \times M$. Then precisely one of the following is true:

- The ends are equal, ie $\left[\Sigma, j, z_{*}, \tilde{u}\right]=\left[\Sigma^{\prime}, j^{\prime}, w_{*}, \tilde{v}\right]$.

- One end is a multiple cover of the other, ie there exists an integer $m \geq 2$ so that either

$$
m \cdot\left[\Sigma, j, z_{*}, \tilde{u}\right]=\left[\Sigma^{\prime}, j^{\prime}, w_{*}, \tilde{v}\right]
$$

or

$$
\left[\Sigma, j, z_{*}, \tilde{u}\right]=m \cdot\left[\Sigma^{\prime}, j^{\prime}, w_{*}, \tilde{v}\right] .
$$

- The ends $\left[\Sigma, j, z_{*}, \tilde{u}\right]$ and $\left[\Sigma^{\prime}, j^{\prime}, w_{*}, \tilde{v}\right]$ are nonintersecting.

Corollary 3.9 Let $\left[\Sigma, j, z_{*}, \tilde{u}\right]$ be a pseudoholomorphic end in $\mathbb{R} \times M$. Then either $\left[\Sigma, j, z_{*}, \tilde{u}\right]$ is embedded or there exists an embedded pseudoholomorphic end $\left[\Sigma^{\prime}, j^{\prime}, w_{*}, \tilde{v}\right]$ and an integer $m \geq 2$ so that

$$
\left[\Sigma, j, z_{*}, \tilde{u}\right]=m \cdot\left[\Sigma^{\prime}, j^{\prime}, w_{*}, \tilde{v}\right] .
$$

Combining these corollaries with results about the local behavior of pseudoholomorphic curves due to Micallef and White [18] or McDuff [17] gives the following generalizations of results that are well-known for closed curves.

The first such result is that two connected curves either have the same image or intersect in at most a finite set.

Corollary 3.10 Let $(W, \bar{J})$ be an almost complex 4-manifold with Hamiltonian structured ends and let $\left[\Sigma_{i}, j_{i}, \Gamma_{i}, \tilde{u}_{i}\right] \in \mathcal{M}\left(W, \bar{J}, \mathcal{H}^{+}, \mathcal{H}^{-}\right)$be connected pseudoholomorphic curves. Then the sets $\tilde{u}_{i}^{-1}\left(\tilde{u}_{j}\left(\Sigma_{j} \backslash \Gamma_{j}\right)\right)$ are either finite or equal to $\Sigma_{j} \backslash \Gamma_{j}$. 
We next address the question of double points and singularities for a connected curve. A pseudoholomorphic curve $[\Sigma, j, \Gamma, \tilde{u}] \in \mathcal{M}\left(W, \bar{J}, \mathcal{H}^{+}, \mathcal{H}^{-}\right)$is called multiply covered it factors through another curve $\left[\Sigma^{\prime}, j^{\prime}, \Gamma^{\prime}, \tilde{v}\right]$ via a holomorphic map $\psi: \Sigma \rightarrow \Sigma^{\prime}$ with $\operatorname{deg} \psi \geq 2$. If a curve is not multiply covered, it is said to be simple. We can now state the following corollary.

Corollary 3.11 Let $(W, \bar{J})$ be an almost complex 4-manifold with Hamiltonian structured ends and let $[\Sigma, j, \Gamma, \tilde{u}] \in \mathcal{M}\left(W, \bar{J}, \mathcal{H}^{+}, \mathcal{H}^{-}\right)$a connected, simple pseudoholomorphic curve. Then the set of double points of $\tilde{u}$

$$
D(\tilde{u})=\left\{(p, q) \in(\Sigma \backslash \Gamma)^{2} \mid \tilde{u}(p)=\tilde{u}(q), p \neq q\right\}
$$

and the set of singular points ${ }^{3}$ of $\tilde{u}$

$$
S(\tilde{u})=\{p \in \Sigma \backslash \Gamma \mid d \tilde{u}(p)=0\}
$$

are both finite.

A version of the following corollary is proved in [10] in the case that the stable Hamiltonian structure arises from a contact form. The proof given there readily generalizes to any stable Hamiltonian structure.

Corollary 3.12 Let $[\Sigma, j, \Gamma, \tilde{u}] \in \mathcal{M}\left(W, \bar{J}, \mathcal{H}^{+}, \mathcal{H}^{-}\right)$be a pseudoholomorphic curve, let $z_{*} \in \Gamma$ be a puncture and let $\pi: T\left(\mathbb{R}^{ \pm} \times M^{ \pm}\right) \rightarrow \xi^{\mathcal{H}^{ \pm}}$be the projection onto $\xi^{\mathcal{H}^{ \pm}}$ over the cylindrical ends determined by the splitting $T\left(\mathbb{R}^{ \pm} \times M^{ \pm}\right) \approx \mathbb{R} \oplus \mathbb{R} X_{\mathcal{H}^{ \pm}} \oplus \xi^{\mathcal{H}^{ \pm}}$. Choose an open neighborhood $U^{\prime} \subset \Sigma$ of $z_{*}$ so that $\tilde{u}\left(U^{\prime} \backslash\left\{z_{*}\right\}\right)$ lies entirely within one of the cylindrical ends so that $\pi \circ d \tilde{u}$ is defined on $U^{\prime} \backslash\left\{z_{*}\right\}$. Then there exists an open neighborhood $U^{\prime} \subset U$ of $z_{*}$ so that $\pi \circ d \tilde{u}$ either vanishes identically on $U^{\prime}$ or vanishes nowhere on $U^{\prime}$.

3.1.4 Asymptotic winding numbers In this section we will define some invariants associated to the ends of an asymptotically cylindrical map. Throughout $(W, \bar{J})$ will denote a 4-dimensional almost complex cobordism with positive/negative ends $\left(\mathbb{R}^{ \pm} \times M^{ \pm}, \widetilde{J}_{ \pm}, \mathcal{H}_{ \pm}=\left(\lambda_{ \pm}, \omega_{ \pm}\right)\right)$.

Let $[\Sigma, j, z,[X], \tilde{u}]$ be a decorated (not necessarily pseudoholomorphic) end which is asymptotically cylindrical over $\gamma^{m}$ for some $\gamma \in \widetilde{\mathcal{P}}^{0}(M, \mathcal{H})$ and some $m \in \mathbb{Z} \backslash\{0\}$. We will abbreviate the end by $[\tilde{u} ; z]$ for simplicity. Define:

$$
\alpha^{\Phi}(\tilde{u} ; z):=\alpha^{\Phi}\left(\gamma^{m}\right) \text { with the right hand side as defined in (3-2). }
$$

${ }^{3}$ Note that the fact that $\tilde{u}$ is pseudoholomorphic implies that either rank $d \tilde{u}(p)=2$ or $d \tilde{u}(p)=0$ for every $p \in \Sigma \backslash \Gamma$. 


$$
\begin{aligned}
& \mu^{\Phi}(\tilde{u} ; z):=\mu^{\Phi}\left(\gamma^{m}\right) \text { with the right hand side as defined in (3-4). } \\
& \bar{\sigma}(\tilde{u} ; z):=\bar{\sigma}\left(\gamma^{m}\right) \text { with the right hand side as defined in (3-9). }
\end{aligned}
$$

We note that each of these quantities depends only on the asymptotic limit of the end and not on the map or the decoration. In the case that the end $[\tilde{u} ; z]$ is pseudoholomorphic and the latter alternative of Theorem 3.7 holds, we define

$$
\begin{gathered}
e_{1}(\tilde{u} ; z):=\text { the leading eigenvector in the asymptotic expression of } \\
\qquad \tilde{u} \text { at } z \text { from Theorem 3.7. }
\end{gathered}
$$

If $|m|>1$ then $e_{1}(\tilde{u} ; z)$ depends on the choice of asymptotic marker, but applying the change of decoration map $\left(e^{i 2 \pi / m}\right) \cdot \in \operatorname{Hom}\left(T_{z} \Sigma\right)$ has the effect of replacing the eigenvector $e_{1}=e_{1}(\tilde{u} ; z)$ with $e_{1}\left(\cdot+\frac{1}{m}\right)$. Thus $e_{1}(\tilde{u} ; z)$ determines a well-defined $m$-fold multisection of $\left.\xi^{\mathcal{H}}\right|_{\gamma}$ which does not depend on the choice of marker.

We say that the end $[\tilde{u} ; z]$ winds if it can be represented by a model $(\Sigma, j, z,[X], \tilde{u}=$ $(a, u))$ in which the map $\tilde{u}$ does not intersect the cylinder over $\gamma^{m}$ or equivalently, so that the projection $u$ of the map to $M$ does not intersect the asymptotic limit $\gamma$. In this case, we define the asymptotic winding $\operatorname{wind}_{\infty}^{\Phi}(\tilde{u} ; z)$ of $\tilde{u}$ at $z$ relative to the trivialization $\Phi$ of $\gamma^{*} \xi^{\mathcal{H}}$ by choosing a small clockwise loop around $z$ and defining wind $_{\infty}^{\Phi}(\tilde{u} ; z)$ to be the winding of the image of this loop around $\gamma$ computed in the trivialization $\Phi$. The assumption that the image of $u$ does not intersect $\gamma$ implies that the resulting quantity is independent of the loop chosen. In the case that $[\tilde{u} ; z]$ is a pseudoholomorphic end, it is an easy consequence of Theorem 3.7 that

$$
\operatorname{wind}_{\infty}^{\Phi}(\tilde{u} ; z):=\operatorname{wind}\left(\Phi^{-1} e_{1}(\tilde{u} ; z)\right)
$$

Note that the comments of the previous paragraph show that $\operatorname{wind}_{\infty}^{\Phi}(\tilde{u} ; z)$ depends only on the undecorated end $[\Sigma, j, z, \tilde{u}]$ and not on the choice of asymptotic marker. The following lemma collects some useful facts about wind $\infty$.

Lemma 3.13 Let $\Phi$ and $\Psi$ be trivializations of $\gamma^{*} \xi^{\mathcal{H}}$ and let $[\tilde{u} ; z]$ denote an end which winds and which is asymptotically cylindrical over $\gamma^{m}$ with $m \in \mathbb{Z} \backslash\{0\}$. Then

$$
\operatorname{wind}_{\infty}^{\Phi}(\tilde{u} ; z)=\operatorname{wind}_{\infty}^{\Psi}(\tilde{u} ; z)+m \operatorname{deg}\left(\Phi^{-1} \circ \Psi\right)
$$

and the quantity

$$
d_{0}(\tilde{u} ; z):=\alpha^{\Phi}(\tilde{u} ; z)-\operatorname{wind}_{\infty}^{\Phi}(\tilde{u} ; z)
$$


is independent of the choice of trivialization. Moreover, if $[\tilde{u} ; z]$ is pseudoholomorphic then $d_{0}(\tilde{u} ; z) \geq 0$ and $^{4}$

$$
\Delta_{1}(\tilde{u} ; z):=(|m|-1) d_{0}(\tilde{u} ; z)-\bar{\sigma}(\tilde{u} ; z)+\operatorname{cov}\left(e_{1}(\tilde{u} ; z)\right)
$$

is even, nonnegative and $\Delta_{1}(\tilde{u} ; z)=0$ if and only if at least one of the following holds:

- $|m|=1$

- $d_{0}(\tilde{u} ; z)=0$

- $d_{0}(\tilde{u} ; z)=1$ and $\bar{\sigma}(\tilde{u} ; z)=|m|$.

Proof The change of trivialization formula follows as in (3-8) and we omit the trivial proof. The trivialization independence of $\alpha^{\Phi}(\tilde{u} ; z)-\operatorname{wind}_{\infty}^{\Phi}(\tilde{u} ; z)$ is then a consequence of the respective change-of-trivialization formulas. In the event that $[\tilde{u} ; z]$ is pseudoholomorphic, the nonnegativity of this quantity has been previously proved in [8] and is an easy consequence the monotonicity of winding relative to eigenvalue (Lemma 3.1) and the fact that $e_{1}(\tilde{u} ; z)$ has negative eigenvalue (Theorem 3.7) and therefore must have winding no greater than $\alpha^{\Phi}(\tilde{u} ; z)$ (by definition of $\alpha$ ).

Next, still assuming that $[\tilde{u} ; z]$ is pseudoholomorphic, we consider the quantity

$$
\Delta_{1}(\tilde{u} ; z)=(|m|-1)\left[\alpha^{\Phi}(\tilde{u} ; z)-\operatorname{wind}_{\infty}^{\Phi}(\tilde{u} ; z)\right]-\bar{\sigma}(\tilde{u} ; z)+\operatorname{cov}\left(e_{1}(\tilde{u} ; z)\right),
$$

and observe that if $\operatorname{wind}_{\infty}^{\Phi}(\tilde{u} ; z)=\alpha^{\Phi}(\tilde{u} ; z)$ then $\operatorname{cov}\left(e_{1}(\tilde{u} ; z)\right)=\bar{\sigma}(\tilde{u} ; z)$ as a consequence of Lemma 3.2, so then $\Delta_{1}(\tilde{u} ; z)=0$. On the other hand, if $|m|=1$, then we must have $\operatorname{cov}\left(e_{1}(\tilde{u} ; z)\right)=\bar{\sigma}(\tilde{u} ; z)=1$ since both of these quantities must divide $m$. Again we have $\Delta_{1}(\tilde{u} ; z)=0$ and we can conclude that $\Delta_{1}(\tilde{u} ; z)=0$ whenever $(|m|-1) d_{0}(\tilde{u} ; z)=0$.

Assuming then that $|m| \geq 2$ and $d_{0}(\tilde{u} ; z) \geq 1$, we get that

$$
\Delta_{1}(\tilde{u} ; z) \geq|m|-1-\bar{\sigma}(\tilde{u} ; z)+\operatorname{cov}\left(e_{1}(\tilde{u} ; z)\right)
$$

which must be nonnegative since $\bar{\sigma}(\tilde{u} ; z) \leq|m|$ while $\operatorname{cov}\left(e_{1}(\tilde{u} ; z)\right) \geq 1$. Moreover, the only possibility for $\Delta_{1}(\tilde{u} ; z)=0$ is if $d_{0}(\tilde{u} ; z)=1, \bar{\sigma}(\tilde{u} ; z)=|m|$ and $\operatorname{cov}\left(e_{1}(\tilde{u} ; z)\right)=$ 1. If we assume that $\bar{\sigma}(\tilde{u} ; z)=|m|$, we can conclude from Lemma 3.2 that

$$
\operatorname{gcd}\left(|m|, \alpha^{\Phi}(\tilde{u} ; z)\right)=\bar{\sigma}(\tilde{u} ; z)=|m|
$$

${ }^{4}$ The significance of the quantity $\Delta_{1}(\tilde{u} ; z)$ is, at this point, likely not apparent to the reader, so we give a brief preview here. As mentioned in the introduction, we will later introduce a quantity $\delta_{\infty}(\tilde{u} ; z)$ for embedded ends which is an upper bound on the number of self-intersections that can appear at that end if pseudoholomorphic. The quantity $\Delta_{1}(\tilde{u} ; z)$ will be twice the contribution to $\delta_{\infty}(\tilde{u}, z)$ (if any) arising from the failure of the leading eigenvector in the asymptotic formula to have extremal winding, ie failure of $d_{0}(\tilde{u} ; z)$ to be zero. 
so $|m|$ divides $\alpha^{\Phi}(\tilde{u} ; z)$. If we further assume that $d_{0}(\tilde{u} ; z)=1$, we can use this with Lemma 3.2 to argue

$$
\begin{aligned}
\operatorname{cov}\left(e_{1}(\tilde{u} ; z)\right) & =\operatorname{gcd}\left(|m|, \operatorname{wind}_{\infty}^{\Phi}(\tilde{u} ; z)\right) \\
& =\operatorname{gcd}\left(|m|, \alpha^{\Phi}(\tilde{u} ; z)-d_{0}(\tilde{u} ; z)\right) \\
& =\operatorname{gcd}\left(|m|, \alpha^{\Phi}(\tilde{u} ; z)-1\right) \\
& =1
\end{aligned}
$$

We can conclude that, if $d_{0}(\tilde{u} ; z)=1$ and $\bar{\sigma}(\tilde{u} ; z)=|m|$, then $\operatorname{cov}\left(e_{1}(\tilde{u} ; z)\right)=1$ and hence $\Delta_{1}(\tilde{u} ; z)=0$. We have thus shown that $\Delta_{1}(\tilde{u} ; z) \geq 0$ and $\Delta_{1}(\tilde{u} ; z)=0$ if and only if $(|m|-1) d_{0}(\tilde{u} ; z)=0$, or $d_{0}(\tilde{u} ; z)=1$ and $\bar{\sigma}(\tilde{u} ; z)=|m|$.

Finally, to prove the evenness of $\Delta_{1}(\tilde{u} ; z)$, we observe that, as a result of Lemma 3.2, the quantity is of the form

$$
(m-1)(a-b)-\operatorname{gcd}(m, a)+\operatorname{gcd}(m, b)
$$

(where here $a=\alpha^{\Phi}(\tilde{u} ; z)$ and $\left.b=\operatorname{wind}_{\infty}^{\Phi}(\tilde{u} ; z)\right)$. If the term $(m-1)(a-b)$ is odd then it must be the case that $m$ is even and $a$ and $b$ have opposite parity. In this case, it follows that $\operatorname{gcd}(m, a)$ and $\operatorname{gcd}(m, b)$ must have opposite parity, so the sum of the three terms is even. In the case that $(m-1)(a-b)$ is even, we have either that $m$ is odd or that $a$ and $b$ have the same parity (or both). In either case, it follows that $\operatorname{gcd}(m, a)$ and $\operatorname{gcd}(m, b)$ have the same parity and so the sum of the three terms must be even.

Next we will introduce a quantity associated to an embedded end which records some of the finer information about the asymptotic behavior in the event that the asymptotic limit of the puncture is multiply covered. Let $[\tilde{u} ; z]=[\Sigma, j, z,[X], \tilde{u}]$ still denote a decorated end in $(\mathbb{R} \times M, \widetilde{J})$ which is asymptotically cylindrical over $\gamma^{m}$, but now assume in addition that $[\tilde{u} ; z]$ is an embedded end and that $|m| \geq 2$. Let $(U, \psi)$ be an asymptotic representative of $[\tilde{u} ; z]$ as defined in the previous section, ie

$$
\tilde{u}(\psi(s, t))=\left(m \tau s, \exp _{\gamma^{m}(t)} U(s, t)\right)
$$

The assumption that $[\tilde{u} ; z]$ is an embedded end implies that $U(s, t)-U\left(s, t+\frac{j}{m}\right)=0$ if and only if $j$ is a multiple of $m$. Consequently, the winding of the map

$$
t \in S^{1} \mapsto \Phi_{\gamma^{m}(t)}^{-1}\left[U(s, t)-U\left(s, t+\frac{j}{m}\right)\right] \in \mathbb{R}^{2} \backslash\{0\}
$$


is well defined and independent of $s$. We define the secondary winding of $\tilde{u}$ at $z$ relative to the trivialization $\Phi$ by

$$
\operatorname{wind}_{2}^{\Phi}(\tilde{u} ; z):=\sum_{j=1}^{|m|-1} \text { wind } \Phi^{-1}\left[U(s, \cdot)-U\left(s, \cdot+\frac{j}{m}\right)\right] .
$$

As with the asymptotic winding, it is straightforward to show that the secondary winding depends only on the undecorated end $[\Sigma, j, z, \tilde{u}]$ and not on the choice of asymptotic marker.

In the event that the embedded end $[\tilde{u} ; z]$ is pseudoholomorphic, the secondary winding can be computed in terms of the eigenfunctions from Theorem 3.6. Letting $(U, \psi)$ still denote an asymptotic representative of $[\tilde{u} ; z]$, we have from Theorem 3.6, that

$$
U(s, t)-U\left(s, t+\frac{j}{m}\right)=e^{\lambda_{j} s}\left[e_{j}(t)+r_{j}(s, t)\right]
$$

with $\lambda_{j}<0$ an eigenvector of $\mathbf{A}=\mathbf{A}_{\gamma^{m}, J}, e_{j} \neq 0$ an eigenvector of $\mathbf{A}$ with eigenvalue $\lambda_{j}$ and $r_{j}$ converging exponentially to 0 . We will write

$$
e_{j}^{\Delta}(\tilde{u} ; z)=e_{j}
$$

to denote the eigenvector appearing in the formula for $U(s, t)-U\left(s+\frac{j}{m}\right)$. Since the $r_{j}$ converge to 0 as $s \rightarrow \infty$, it follows that

$$
\operatorname{wind}_{2}^{\Phi}(\tilde{u} ; z)=\sum_{j=1}^{|m|-1} \operatorname{wind}\left(\Phi^{-1} e_{j}^{\Delta}(\tilde{u} ; z)\right)
$$

We collect some useful properties of wind 2 in the following lemma.

Lemma 3.14 Let $[\tilde{u}, z]=[\Sigma, j, z, \tilde{u}]$ be an embedded end in $(\mathbb{R} \times M, \widetilde{J})$ and assume that $[\tilde{u} ; z]$ is asymptotically cylindrical over $\gamma^{m}$. If $\Phi$ and $\Psi$ are trivializations of $\gamma^{*} \xi^{\mathcal{H}}$ then

$$
\operatorname{wind}_{2}^{\Phi}(\tilde{u} ; z)=\operatorname{wind}_{2}^{\Psi}(\tilde{u} ; z)+m(|m|-1) \operatorname{deg}\left(\Phi^{-1} \circ \Psi\right) .
$$

If $[\tilde{u} ; z]$ is pseudoholomorphic, then the quantities ${ }^{5}$

$$
\Delta_{2}(\tilde{u} ; z):=(|m|-1) \operatorname{wind}_{\infty}^{\Phi}(\tilde{u} ; z)-\operatorname{cov}\left(e_{1}(\tilde{u} ; z)\right)+1-\operatorname{wind}_{2}^{\Phi}(\tilde{u} ; z)
$$

${ }^{5}$ As with $\Delta_{1}(\tilde{u} ; z)$ before, the significance of the quantities $\Delta_{2}(\tilde{u} ; z)$ and $\Delta_{t o t}(\tilde{u} ; z)$ is likely not apparent to the reader at this point, so we give a brief preview here. As mentioned before, we will later introduce a quantity $\delta_{\infty}(\tilde{u} ; z)$ for embedded ends which is an upper bound on the number of selfintersections that can appear at that end if pseudoholomorphic. The quantity $\Delta_{2}(\tilde{u} ; z)$ will be twice the contribution to $\delta_{\infty}(\tilde{u}, z)$ (if any) arising from the failure of the eigenvectors in the asymptotic formula from Theorem 3.7 to be simply covered, while $\Delta_{t o t}(\tilde{u} ; z)$ is the sum of $\Delta_{1}(\tilde{u} ; z)$ and $\Delta_{2}(\tilde{u} ; z)$. 


$$
\Delta_{t o t}(\tilde{u} ; z)=(|m|-1) \alpha^{\Phi}(\tilde{u} ; z)-\bar{\sigma}(\tilde{u} ; z)+1-\operatorname{wind}_{2}^{\Phi}(\tilde{u} ; z)
$$

are even, nonnegative and independent of the choice of trivialization. Moreover, we have that $\Delta_{2}(\tilde{u} ; z)=0$ if and only if the asymptotic representation of $\tilde{u}$ near $z$ (from Theorem 3.7) has at most two terms and the winding of the eigenvectors appearing in this formula differ precisely by 1 . In particular

$$
\operatorname{wind}_{2}^{\Phi}(\tilde{u} ; z)=(|m|-1) \operatorname{wind}_{\infty}^{\Phi}(\tilde{u} ; z)
$$

if and only if $\operatorname{cov}\left(e_{1}(\tilde{u} ; z)\right)=1$.

Proof The change of trivialization formula follows as in (3-8) and we again omit the easy proof. The trivialization independence of the two quantities $\Delta_{2}(\tilde{u} ; z)$ and $\Delta_{\text {tot }}(\tilde{u} ; z)$ is then a straightforward consequence of the change of trivialization formulas.

Assuming now that $[\tilde{u} ; z]$ is pseudoholomorphic, the nonnegativity and evenness of $\Delta_{\text {tot }}(\tilde{u} ; z)$ will follow from the nonnegativity and evenness of $\Delta_{2}(\tilde{u} ; z)$ along with Lemma 3.13 , since $\Delta_{\text {tot }}(\tilde{u} ; z)-\Delta_{2}(\tilde{u} ; z)=\Delta_{1}(\tilde{u} ; z)$, which was already shown to be nonnegative and even.

To prove that $\Delta_{2}(\tilde{u} ; z)$ is even and nonnegative, we first write down an alternate formula for wind $_{2}$ in terms of the eigenvectors appearing in Theorem 3.7. According to the theorem, we can write

$$
\tilde{u}(\psi(s, t))=\left(m \tau s, \exp _{\gamma^{m}(t)} \sum_{i=1}^{N} e^{\lambda_{i} s}\left[e_{i}(t)+r_{i}(s, t)\right]\right)
$$

with $\lambda_{i}<\lambda_{i-1}$, the sequence

$$
k_{1}=\operatorname{cov}\left(e_{1}\right) \quad k_{i}=\operatorname{gcd}\left(k_{i-1}, \operatorname{cov}\left(e_{i}\right)\right)
$$

strictly decreasing in $i$ and the $r_{i}$ satisfying $r_{i}\left(s, t+\frac{1}{k_{i}}\right)=r_{i}(s, t)$ and converging exponentially to zero. We observe that Lemma 3.1 implies that wind $\Phi^{-1} e_{i} \leq$ wind $\Phi^{-1} e_{i-1}$ since $\lambda_{i}<\lambda_{i-1}$. Since $k_{i-1}$ divides $\operatorname{cov}\left(e_{i-1}\right)$ and $\operatorname{gcd}\left(k_{i-1}, \operatorname{cov}\left(e_{i}\right)\right)=k_{i}<k_{i-1}$ it follows that $\operatorname{cov}\left(e_{i}\right) \neq \operatorname{cov}\left(e_{i-1}\right)$, so Lemma 3.2 lets us conclude that we have the strict inequality wind $\Phi^{-1} e_{i}<$ wind $\Phi^{-1} e_{i-1}$.

Abbreviating $k_{0}=|m|$ and $w_{i}=$ wind $\Phi^{-1} e_{i}$, we claim that we can conclude from (3-23) that

$$
\operatorname{wind}_{2}^{\Phi}(\tilde{u} ; z)=\sum_{i=1}^{N}\left(k_{i-1}-k_{i}\right) w_{i}
$$


To see this, observe that if $e_{i}(\cdot)-e_{i}\left(\cdot+\frac{j}{k_{0}}\right)$ is nonzero then Lemma 3.1 implies that

$$
\text { wind } \Phi^{-1} e_{i}=\text { wind } \Phi^{-1}\left[e_{i}(\cdot)-e_{i}\left(\cdot+\frac{j}{k_{0}}\right)\right]
$$

since $e_{i}$ and $e_{i}(\cdot)-e_{i}\left(\cdot+\frac{j}{k_{0}}\right)$ are eigenvectors of $\mathbf{A}$ with the same eigenvalue. Then, we can use that $r_{i}\left(s, t+\frac{1}{k_{i}}\right)=r_{i}(s, t)$ and that

$$
e_{i}\left(t+\frac{1}{k_{i}}\right)=e_{i}\left(t+\frac{\operatorname{cov}\left(e_{i}\right) / k_{i}}{\operatorname{cov}\left(e_{i}\right)}\right)=e_{i}(t)
$$

to conclude that if $(U, \psi)$ is an asymptotic representative of $\tilde{u}$, then as $j$ varies over $\left\{1, \ldots, k_{0}-1\right\}$, precisely $k_{i-1}-k_{i}$ of the terms $\Phi^{-1}\left[U(s, \cdot)-U\left(s, \cdot+\frac{j}{k_{0}}\right)\right]$ have winding $w_{i}$ for $s$ large. The formula (3-24) follows immediately from this observation and the definition of wind $_{2}$.

Using the formula (3-24), the quantity $\Delta_{2}(\tilde{u} ; z)$ of interest can be written

$$
\Delta_{2}(\tilde{u} ; z)=\left(k_{0}-1\right) w_{1}-k_{1}+1-\sum_{i=1}^{N}\left(k_{i-1}-k_{i}\right) w_{i} .
$$

Using that $k_{N}=1$ since $[u ; z]$ is an embedded end, we can rewrite this as

$$
\begin{aligned}
\Delta_{2}(\tilde{u} ; z) & =\sum_{i=1}^{N}\left(k_{i-1}-k_{i}\right) w_{1}-k_{1}+1-\sum_{i=1}^{N}\left(k_{i-1}-k_{i}\right) w_{i} \\
& =1-k_{1}+\sum_{i=1}^{N}\left(k_{i-1}-k_{i}\right)\left(w_{1}-w_{i}\right)
\end{aligned}
$$

and since the $i=1$ term of the sum vanishes, we continue

$$
\begin{aligned}
& =1-k_{1}+\sum_{i=2}^{N}\left(k_{i-1}-k_{i}\right)\left(w_{1}-w_{i}\right) \\
& =1-k_{1}+\sum_{i=2}^{N} \sum_{j=2}^{i}\left(k_{i-1}-k_{i}\right)\left(w_{j-1}-w_{j}\right) \\
& =1-k_{1}+\sum_{j=2}^{N} \sum_{i=j}^{N}\left(k_{i-1}-k_{i}\right)\left(w_{j-1}-w_{j}\right) \\
& =1-k_{1}+\sum_{j=2}^{N}\left(k_{j-1}-1\right)\left(w_{j-1}-w_{j}\right)
\end{aligned}
$$




$$
=\sum_{j=2}^{N}\left(k_{j-1}-1\right)\left(w_{j-1}-w_{j}\right)+k_{j}-k_{j-1} .
$$

Using that $w_{j-1}-w_{j} \geq 1$ for each $j$ and that the $k_{j}$ are a decreasing sequence of positive integers, we find that each term in this sum in nonnegative (in fact positive except possibly when $j=N$ ). Using the definition of $k_{j}$ with Lemma 3.2, we have that

$$
k_{j}=\operatorname{gcd}\left(k_{j-1}, \operatorname{cov}\left(e_{j}(\tilde{u} ; z)\right)\right)=\operatorname{gcd}\left(k_{j-1}, \operatorname{gcd}\left(k_{0}, w_{j}\right)\right)=\operatorname{gcd}\left(k_{j-1}, w_{j}\right)
$$

since $k_{j-1}$ divides $k_{0}$. Using further that $k_{j}$ divides $w_{j}$, we can also write $k_{j}=$ $\operatorname{gcd}\left(k_{j}, w_{j}\right)$. These observations allow us to rewrite this sum as

$$
\sum_{j=2}^{N}\left(k_{j-1}-1\right)\left(w_{j-1}-w_{j}\right)+\operatorname{gcd}\left(k_{j-1}, w_{j}\right)-\operatorname{gcd}\left(k_{j-1}, w_{j-1}\right) .
$$

Comparing this with (3-20), it easily follows that each term in the sum is even and consequently that the sum is even.

Finally, to see the last claims are true, we observe that it follows from (3-25) that $\Delta_{2}(\tilde{u} ; z)=0$ if and only if $w_{j-1}-w_{j}=1$ and $k_{j}=1$ for all values of $j \in \mathbb{Z} \cap[2, N]$ for which they are defined. Since the $k_{j}$ form a decreasing sequence of positive integers, we conclude that $\Delta_{2}(\tilde{u} ; z)=0$ precisely when either $k_{2}=1$ and $w_{1}-w_{2}=1$ or when $k_{1}=1$, ie precisely when the asymptotic representation (3-23) has at most two terms with the winding of the two eigenvectors (in the case of two terms) appearing in that formula differing by 1 . Moreover, there is one term in this representation if and only if $k_{1}=1$, which happens precisely when the leading eigenvector $e_{1}(\tilde{u} ; z)$ is simply covered, and in this case $\Delta_{2}(\tilde{u} ; z)=0$ is equivalent to

$$
\operatorname{wind}_{2}^{\Phi}(\tilde{u} ; z)=(|m|-1) \operatorname{wind}_{\infty}^{\Phi}(\tilde{u} ; z) .
$$

We close this section by defining a quantity that records relative asymptotic information about two nonintersecting ends limiting to a cover of the same orbit with the same covering number. We again let $[\tilde{u} ; z]=[\Sigma, j, z,[X], \tilde{u}]$ be a decorated end which is asymptotically cylindrical over $\gamma^{m}$, we let $[\tilde{v} ; w]=\left[\Sigma^{\prime}, j^{\prime}, w,[X], \tilde{v}\right]$ be a second decorated end which is also asymptotically cylindrical over $\gamma^{m}$ and assume that the end $[\tilde{u} ; z]$ and $[\tilde{v} ; w]$ are nonintersecting. If we let $(U, \psi)$ and $(V, \phi)$ be asymptotic representatives of $[\tilde{u} ; z]$ and $[\tilde{v}, w]$ respectively, then the assumption that $[\tilde{u} ; z]$ and $[\tilde{v} ; w]$ are nonintersecting implies that $U(s, t)-V\left(s, t+\frac{j}{m}\right)$ is never zero. Consequently, the maps

$$
t \in S^{1} \mapsto \Phi_{\gamma^{m}(t)}^{-1}\left[U(s, t)-V\left(s, t+\frac{j}{m}\right)\right] \in \mathbb{R}^{2} \backslash\{0\}
$$


have well-defined winding which is independent of $s$. We then define the total relative winding of $[\tilde{u} ; z]$ and $[\tilde{v} ; w]$ relative to a trivialization $\Phi$ of $\gamma^{*} \xi^{\mathcal{H}}$ by

$$
\operatorname{wind}_{\mathrm{rel}}^{\Phi}([\tilde{u} ; z],[\tilde{v} ; w]):=\sum_{j=0}^{|m|-1} \operatorname{wind} \Phi^{-1}\left[U(s, \cdot)-V\left(s, \cdot+\frac{j}{m}\right)\right] .
$$

As with the secondary winding, the total relative winding of two nonintersecting ends is easily verified to be independent of choice of asymptotic marker and therefore gives a well-defined invariant of the pair of undecorated ends $[\Sigma, j, z, \tilde{u}]$ and $\left[\Sigma^{\prime}, j^{\prime}, w, \tilde{v}\right]$.

In the event that the ends $[\tilde{u} ; z]$ and $[\tilde{v} ; w]$ are pseudoholomorphic, the total relative winding can be computed in terms of the eigenfunctions appearing in Theorem 3.6. Indeed the assumption that $[\tilde{u} ; z]$ and $[\tilde{v} ; w]$ are nonintersecting tells us that for each $j \in\{0, \ldots,|m|-1\}$ we can write

$$
U(s, t)-V\left(s, t+\frac{j}{k}\right)=e^{\lambda_{j} s}\left[e_{j}(t)+r_{j}(s, t)\right]
$$

where $e_{j}$ is an eigenvector of the asymptotic operator with eigenvalue $\lambda_{j}<0$ and where $r_{j}$ converges exponentially to zero. The fact that the $r_{j}$ converge to zero as $s \rightarrow \infty$ lets us conclude that

$$
\operatorname{wind}_{\mathrm{rel}}^{\Phi}([\tilde{u} ; z],[\tilde{v} ; w])=\sum_{j=0}^{|m|-1} \operatorname{wind}\left(\Phi^{-1} e_{j}\right) .
$$

We collect some useful properties of wind rel $_{\text {in }}$ ine following lemma.

Lemma 3.15 Let $[\tilde{u} ; z]=[\Sigma, j, z, \tilde{u}]$ and $[\tilde{v} ; w]=\left[\Sigma^{\prime}, j^{\prime}, w, \tilde{v}\right]$ be nonintersecting ends in $(\mathbb{R} \times M, \widetilde{J})$ which are asymptotically cylindrical over $\gamma^{m}$. If $\Phi$ and $\Psi$ are trivializations of $\gamma^{*} \xi^{\mathcal{H}}$, then

$$
\operatorname{wind}_{\mathrm{rel}}^{\Phi}([\tilde{u} ; z],[\tilde{v} ; w])=\operatorname{wind}_{\mathrm{rel}}^{\Psi}([\tilde{u} ; z],[\tilde{v} ; w])+m|m| \operatorname{deg}\left(\Phi^{-1} \circ \Psi\right)
$$

and

$$
\operatorname{wind}_{\mathrm{rel}}^{\Phi}([\tilde{u} ; z],[\tilde{v} ; w])=\operatorname{wind}_{\mathrm{rel}}^{\Phi}([\tilde{v} ; w],[\tilde{u} ; z]) .
$$

If $[\tilde{u} ; z]$ and $[\tilde{v} ; w]$ are pseudoholomorphic ends, the quantity

$$
|m| \max \left\{\operatorname{wind}_{\infty}^{\Phi}(\tilde{u} ; z), \operatorname{wind}_{\infty}^{\Phi}(\tilde{v} ; w)\right\}-\operatorname{wind}_{\operatorname{rel}}^{\Phi}([\tilde{u} ; z],[\tilde{v} ; w])
$$

is nonnegative and independent of choice of trivialization and we have the strict inequality

$$
|m| \max \left\{\operatorname{wind}_{\infty}^{\Phi}(\tilde{u} ; z), \operatorname{wind}_{\infty}^{\Phi}(\tilde{v} ; w)\right\}-\operatorname{wind}_{\mathrm{rel}}^{\Phi}([\tilde{u} ; z],[\tilde{v} ; w])>0
$$


only if $e_{1}(\tilde{u} ; z)=j *_{m} e_{1}(\tilde{v} ; w)$ for some $j \in \mathbb{Z}_{|m|}$, where $*_{m}$ denotes the $\mathbb{Z}_{|m|}$-action on $\left(\gamma^{m}\right)^{*} \xi^{\mathcal{H}}$ (or equivalently if $e_{1}(\tilde{u} ; z)$ and $e_{1}(\tilde{v} ; w)$ determine the same multisection of $\left.\gamma^{*} \xi^{\mathcal{H}}\right)$.

Proof As with Lemma 3.13 and Lemma 3.14, the change of trivialization formula follows just as in (3-8) and we omit the trivial proof. The symmetry of the total relative winding is an easy consequence of the fact that winding is fixed by negation and the $\mathbb{Z}_{m}$ action, while the fact that the quantity

$$
|m| \max \left\{\operatorname{wind}_{\infty}^{\Phi}(\tilde{u} ; z), \operatorname{wind}_{\infty}^{\Phi}(\tilde{v} ; w)\right\}-\operatorname{wind}_{\mathrm{rel}}^{\Phi}([\tilde{u} ; z],[\tilde{v} ; w])
$$

is independent of choice of trivialization follows from the change-of-trivialization formulas for wind rel $_{\text {and }}$ wind $_{\infty}$.

Now assume that $[\tilde{u} ; z]$ and $[\tilde{v} ; w]$ are pseudoholomorphic. To see that

$$
|m| \max \left\{\operatorname{wind}_{\infty}^{\Phi}(\tilde{u} ; z), \operatorname{wind}_{\infty}^{\Phi}(\tilde{v} ; w)\right\}-\operatorname{wind}_{\mathrm{rel}}^{\Phi}([\tilde{u} ; z],[\tilde{v} ; w]) \geq 0
$$

we observe that the differences

$$
U(s, t)-V\left(s, t+\frac{j}{k}\right)
$$

cannot decay slower than both of $U$ and $V$. This observation with the definition of wind $_{\text {rel }}$ and the monotonicity of winding with respect to eigenvalue imply that

$$
\operatorname{wind}_{\mathrm{rel}}^{\Phi}([\tilde{u} ; z],[\tilde{v} ; w]) \leq|m| \max \left\{\operatorname{wind}_{\infty}^{\Phi}(\tilde{u} ; z), \operatorname{wind}_{\infty}^{\Phi}(\tilde{v} ; w)\right\}
$$

as claimed. To prove the final claim, we first assume that $e_{1}(\tilde{u} ; z)$ and $e_{1}(\tilde{v} ; w)$ have different eigenvalues. Using the symmetry of the relative winding, we can assume without loss of generality that the eigenvalue of $e_{1}(\tilde{u} ; z)$ is strictly larger than that of $e_{1}(\tilde{v} ; w)$. In this case, it is immediate that $e_{1}(\tilde{u} ; z)$ must be the eigenvector appearing in the asymptotic formula for $U(s, t)-V\left(s, t+\frac{j}{m}\right)$ for all $j \in \mathbb{Z}_{m}$. It follows from the definition of the total relative winding and the monotonicity of winding in the eigenvalue (Lemma 3.1) that

$$
\operatorname{wind}_{\mathrm{rel}}^{\Phi}([\tilde{u} ; z],[\tilde{v} ; w])=|m| \operatorname{wind}_{\infty}^{\Phi}(\tilde{u} ; z)=|m| \max \left\{\operatorname{wind}_{\infty}^{\Phi}(\tilde{u} ; z), \operatorname{wind}_{\infty}^{\Phi}(\tilde{v} ; w)\right\}
$$

as claimed. Next assuming that $e_{1}(\tilde{u} ; z)$ and $e_{1}(\tilde{v} ; w)$ have the same eigenvalue (and hence the same winding), but belong to different $\mathbb{Z}_{m}$-orbits. Then it is again straightforward to argue that the eigenvector appearing in the asymptotic formula of $U(s, t)-V\left(s, t+\frac{j}{m}\right)$ is

$$
e_{1}(\tilde{u} ; z)-j *_{m} e_{1}(\tilde{v} ; w)
$$


and hence

$$
\begin{aligned}
\operatorname{wind}_{\mathrm{rel}}^{\Phi}([\tilde{u} ; z],[\tilde{v} ; w]) & =\sum_{j=0}^{|m|-1} \operatorname{wind} \Phi^{-1}\left[e_{1}(\tilde{u} ; z)-j *_{m} e_{1}(\tilde{v} ; w)\right] \\
& =|m| \operatorname{wind}_{\infty}^{\Phi}(\tilde{u} ; z)\left(=|m| \operatorname{wind}_{\infty}^{\Phi}(\tilde{v} ; w)\right) \\
& =|m| \max \left\{\operatorname{wind}_{\infty}^{\Phi}(\tilde{u} ; z), \operatorname{wind}_{\infty}^{\Phi}(\tilde{v} ; w)\right\} .
\end{aligned}
$$

The only remaining possibility is that $e_{1}(\tilde{u} ; z)$ and $e_{1}(\tilde{v} ; w)$ have the same eigenvalue and that $e_{1}(\tilde{u} ; z)$ is in the $\mathbb{Z}_{m}$-orbit of $e_{1}(\tilde{v} ; w)$. Hence, this must be true if the strict inequality is to occur.

\subsection{Local intersection theory at $\infty$}

We now study local intersection properties of asymptotically cylindrical maps near their punctures. The key idea, considered in a special case by Kriener in [16] and more generally by Hutchings in [14], is to perturb the maps near the ends and compute an intersection number which depends on the direction of the perturbation. We will see that for pseudoholomorphic curves, this intersection number is always bounded from below by a number which also depends on the direction of perturbation, but that the difference between the intersection number and its lower bound is independent of this choice. We can thus associate to each pseudoholomorphic end or pair of ends, an "asymptotic self-intersection/intersection number" which, roughly speaking, measures tangencies (or self-tangencies) at infinity. Many of our results in this section are analogous to those in [14, Section 6]. We present full proofs here because our point of view and conventions are somewhat different than those used in [14] and because in one case we achieve a stronger bound than what is given in [14] which is important for our applications.

We start by considering an embedded (not necessarily pseudoholomorphic) end model $(\Sigma, j, z, \tilde{u})$ in $\mathbb{R} \times M$, which we assume is asymptotically cylindrical over $\gamma^{m}$. Let $(U, \psi)$ denote an asymptotic representative for $\tilde{u}$, so that

$$
\tilde{u}(\psi(s, t))=\left(m \tau s, \exp _{\gamma^{m}(t)} U(s, t)\right)
$$

with $\psi:[R, \infty) \times S^{1} \rightarrow \Sigma \backslash\{z\}$ an embedding converging to $z$ as $s \in[R, \infty)$ approaches infinity.

Letting $\Phi: S^{1} \times \mathbb{C} \rightarrow \gamma^{*} \xi^{\mathcal{H}}$ be a unitary trivialization of $\gamma^{*} \xi^{\mathcal{H}}$ and $\varepsilon \in \mathbb{R}^{+} \subset \mathbb{C}$ be a positive real number (thought of as lying in $\mathbb{C}$ ), we define a map $\tilde{u}_{z, \varepsilon, \Phi}:[R, \infty) \times S^{1} \rightarrow$ $\mathbb{R} \times M$ by

$$
\tilde{u}_{z, \varepsilon, \Phi}(s, t)=\left(m \tau s, \exp _{\gamma^{m}(t)}[U(s, t)+\Phi(m t) \varepsilon]\right)
$$


so that $\tilde{u}_{z, \varepsilon, \Phi}$ is a perturbation of $\tilde{u}$ near $z$ in a direction determined by $\Phi$.

Lemma 3.16 There exists an $\varepsilon_{0}>0$ so that the intersection number

$$
\operatorname{int}\left(\left.\tilde{u}\right|_{\psi\left([R, \infty) \times S^{1}\right)}, \tilde{u}_{z, \varepsilon, \Phi}\right)
$$

is well-defined, independent of $\varepsilon \in\left(0, \varepsilon_{0}\right)$ and invariant under homotopies $\Phi_{\tau}: S^{1} \times$ $\mathbb{C} \rightarrow \gamma^{*} \xi^{\mathcal{H}}$ of unitary trivializations of $\gamma^{*} \xi^{\mathcal{H}}$.

Proof Abbreviating $C_{R}=[R, \infty) \times S^{1}$ and $I_{R}=[R, R+1] \times S^{1}$, it follows from the asymptotic behavior of $\tilde{u}$ and the assumption that $\left.\tilde{u}\right|_{\psi\left([R, \infty) \times S^{1}\right)}$ is an embedding that we can find a number $\varepsilon_{0}>0$ so that for any $\varepsilon \in\left(0, \varepsilon_{0}\right)$ there exists an $R(\varepsilon)$ so that

$\tilde{u}\left(\psi\left(I_{R} \cup C_{R(\varepsilon)}\right)\right) \cap \tilde{u}_{z, \varepsilon, \Phi}\left(C_{R}\right)=\varnothing \quad$ and $\quad \tilde{u}\left(\psi\left(C_{R}\right)\right) \cap \tilde{u}_{z, \varepsilon, \Phi}\left(I_{R} \cup C_{R(\varepsilon)}\right)=\varnothing$

for any unitary trivialization $\Phi$; that is, the preimages of all intersections of $\tilde{u}$ and $\tilde{u}_{z, \varepsilon, \Phi}$ are contained in the cylinder $[R+1, R(\varepsilon)] \times S^{1}$.

With this condition satisfied, we can apply standard transversality arguments to find smooth homotopies $v ., w .:[0,1] \times[R, \infty) \times S^{1} \rightarrow \mathbb{R} \times M$ so that $v_{0}=\tilde{u} \circ \psi$ and $w_{0}=\tilde{u}_{z, \varepsilon, \Phi}$

$$
\begin{array}{lll}
\left.v_{\tau}\right|_{I_{R} \cup C_{R(\varepsilon)}}=\left.\tilde{u} \circ \psi\right|_{I_{R} \cup C_{R(\varepsilon)}} & \text { and } & \left.w_{\tau}\right|_{I_{R} \cup C_{R(\varepsilon)}}=\left.\tilde{u}_{z, \varepsilon, \Phi}\right|_{I_{R} \cup C_{R(\varepsilon)}}, \\
v_{\tau}\left(I_{R} \cup C_{R(\varepsilon)}\right) \cap w_{\tau}\left(C_{R}\right)=\varnothing & \text { and } & v_{\tau}\left(C_{R}\right) \cap w_{\tau}\left(I_{R} \cup C_{R(\varepsilon)}\right)=\varnothing
\end{array}
$$

for all $\tau \in[0,1]$ and so that $v_{1}$ and $w_{1}$ have only transverse intersections. These conditions imply that the set $S=\left\{(p, q) \in C_{R} \times C_{R} \mid v_{1}(p)=w_{1}(q)\right\}$ is contained in $\left([R+1, R(\varepsilon)] \times S^{1}\right)^{2}$ and the transversality assumption implies that the set $S$ is finite. We can therefore define the intersection number $\operatorname{int}\left(v_{1}, w_{1}\right)$ by counting with sign the intersections of $v_{1}$ and $w_{1}$ and we define

$$
\operatorname{int}\left(\left.\tilde{u}\right|_{\psi\left([R, \infty) \times S^{1}\right)}, \tilde{u}_{z, \varepsilon, \Phi}\right)=\operatorname{int}\left(v_{1}, w_{1}\right) .
$$

Considering a generic path of homotopies shows that this number does not depend on the choice of homotopy. Moreover, if $\tilde{u}_{z}^{\tau}$ and $\tilde{u}_{z, \varepsilon, \Phi}^{\tau}$ are any homotopies starting at $\left.\tilde{u}\right|_{\psi\left([R, \infty) \times S^{1}\right)}$ and $\tilde{u}_{z, \varepsilon, \Phi}$ respectively, we will have that

$$
\operatorname{int}\left(\left.\tilde{u}\right|_{\psi\left([R, \infty) \times S^{1}\right)}, \tilde{u}_{z, \varepsilon, \Phi}\right)=\operatorname{int}\left(\tilde{u}_{z}^{\tau}, \tilde{u}_{z, \varepsilon, \Phi}^{\tau}\right)
$$

provided that there exists an $R_{1}$ so that

$$
\tilde{u}_{z}^{\tau}\left(I_{R} \cup C_{R_{1}}\right) \cap \tilde{u}_{z, \varepsilon, \Phi}^{\tau}\left(C_{R}\right)=\varnothing \quad \text { and } \quad \tilde{u}_{z}^{\tau}\left(C_{R}\right) \cap \tilde{u}_{z, \varepsilon, \Phi}^{\tau}\left(I_{R} \cup C_{R_{1}}\right)=\varnothing
$$


for all $\tau \in[0,1]$. In particular, this homotopy invariance implies that the intersection number $\operatorname{int}\left(\left.\tilde{u}\right|_{\psi\left([R, \infty) \times S^{1}\right)}, \tilde{u}_{z, \varepsilon, \Phi}\right)$ is independent of $\varepsilon \in\left(0, \varepsilon_{0}\right)$ and invariant under homotopies of unitary trivializations $\Phi_{\tau}: S^{1} \times \mathbb{C} \rightarrow \gamma^{*} \xi^{\mathcal{H}}$.

We use the above lemma to define an invariant of an embedded, asymptotically cylindrical end model. Let $(\Sigma, j, z, \tilde{u})$ still denote an embedded end model, let $O$ be some open neighborhood of $z$ and let $\tilde{u}_{z, \varepsilon, \Phi}$ denote a perturbation of $\left.\tilde{u}\right|_{O}$ near $z$ defined as above. The preceding lemma shows that the intersection number

$$
\operatorname{int}\left(\left.\tilde{u}\right|_{O}, \tilde{u}_{z, \varepsilon, \Phi}\right)
$$

is independent of sufficiently small $\varepsilon>0$ and depends only on the map $\tilde{u}$ and the homotopy class of unitary trivialization $\Phi$. Using this observation, we define the relative asymptotic self-intersection number $i_{\infty}^{\Phi}(\tilde{u} ; z)$ of the end model $(\Sigma, j, z, \tilde{u})$ relative to $\Phi$ by

$$
i_{\infty}^{\Phi}(\tilde{u} ; z):=\operatorname{int}\left(\left.\tilde{u}\right|_{O}, \tilde{u}_{z, \varepsilon, \Phi}\right)
$$

for any sufficiently small $\varepsilon>0$ so that the conclusions of the preceding lemma hold.

Using Theorem 3.7, we can compute $i_{\infty}^{\Phi}(\tilde{u} ; z)$ in terms of the winding invariants defined in the previous section. We state this as a lemma.

Lemma 3.17 Let $(\Sigma, j, z, \tilde{u})$ be an embedded, asymptotically cylindrical end model and let $\Phi$ be a unitary trivialization of the plane-field $\xi^{\mathcal{H}}$ along the asymptotic limit of $\tilde{u}$ at $z$. Then the asymptotic self-intersection number $i_{\infty}^{\Phi}(\tilde{u} ; z)$ of $\tilde{u}$ at $z$ relative to $\Phi$ is given by

$$
i_{\infty}^{\Phi}(\tilde{u} ; z)=-\operatorname{wind}_{2}^{\Phi}(\tilde{u} ; z) .
$$

We remark that this Lemma demonstrates that the asymptotic intersection number is an invariant of the embedded pseudoholomorphic end $[\Sigma, j, z, \tilde{u}]$ and does not depend on the choice of embedded model $(\Sigma, j, z, \tilde{u})$ representing $[\Sigma, j, z, \tilde{u}]$.

Proof Assume that $\gamma^{m}$ is the asymptotic limit of the end and let $(U, \psi)$ be an asymptotic representative of the end, so we can write

$$
\tilde{u}(\psi(s, t))=\left(m \tau s, \exp _{\gamma^{m}(t)} U(s, t)\right)
$$

Note that the assumption that $\tilde{u}$ is an embedding implies $U(s, t)-U\left(s, t+\frac{j}{m}\right)$ has no zeroes for $j \neq 0 \bmod m$. By definition, the asymptotic self-intersection number $i_{\infty}^{\Phi}(\tilde{u} ; z)$ is computed by counting signed intersections of $\tilde{u}$ and the map

$$
\tilde{u}_{z, \varepsilon, \Phi}(s, t)=\left(m \tau s, \exp _{\gamma^{m}(t)}[U(s, t)+\Phi(m t) \varepsilon]\right) .
$$


Considering the representations given for these maps, we see that

if and only if

$$
\tilde{u} \circ \psi(s, t)=\tilde{u}_{z \cdot \varepsilon, \Phi}\left(s^{\prime}, t^{\prime}\right)
$$

$$
\begin{gathered}
s=s^{\prime}, \\
t=t^{\prime}+\frac{j}{m} \quad \text { for some } \quad j \in \mathbb{Z}_{m} \quad \text { and } \\
U(s, t)=U\left(s, t+\frac{j}{m}\right)+\Phi(m t) \varepsilon,
\end{gathered}
$$

where in the last condition we have applied the previous two and have used the 1periodicity of $\Phi$ and the $\frac{1}{m}$-periodicity of $\gamma^{m}$. It clear then that the algebraic count of intersections of $\tilde{u}$ with $\tilde{u}_{z, \varepsilon, \Phi}$ is equal to the sum of the algebraic count of zeroes of

$$
U(s, t)-U\left(s, t+\frac{j}{m}\right)-\Phi(m t) \varepsilon
$$

as $j$ varies over $\mathbb{Z}_{m}$, which in turn is equal to the algebraic count of zeroes of the function $F_{j}:[R, \infty) \times S^{1} \rightarrow \mathbb{C}$ defined by

$$
F_{j}(s, t)=\Phi(m t)^{-1}\left[U(s, t)-U\left(s, t+\frac{j}{m}\right)\right]-\varepsilon \in \mathbb{C}
$$

as $j$ varies over $\mathbb{Z}_{m} \backslash\{0\}$.

Since all zeroes of the $F_{j}$ lie in the interior of a compact cylinder of the form $\left[R_{1}, R_{2}\right] \times$ $S^{1}$, the algebraic count of zeroes of $F_{j}$ will be equal to the difference in winding of the loop $t \mapsto F_{j}(s, t)$ for $s \geq R_{2}$ and $s \leq R_{1}$. For large $s^{\prime} \geq R_{2}$, the assumed decay of $U-j * U$ implies that

$$
\operatorname{wind}\left(F_{j}\left(s^{\prime}, \cdot\right)\right)=\operatorname{wind}\left(\Phi(m \cdot)^{-1}\left[U(s, \cdot)-U\left(s, \cdot+\frac{j}{m}\right)\right]-\varepsilon\right)=\operatorname{wind}(-\varepsilon)=0 .
$$

Next, assuming we have chosen $\varepsilon<\inf _{t \in S^{1}}\left|U\left(R_{1}, t\right)-U\left(R_{1}, t+\frac{j}{m}\right)\right|$, we have that

$$
\begin{aligned}
\operatorname{wind}\left(F_{j}\left(R_{1}, \cdot\right)\right) & =\operatorname{wind}\left(\Phi(m \cdot)^{-1}\left[U\left(R_{1}, \cdot\right)-U\left(R_{1}, \cdot+\frac{j}{m}\right)\right]-\varepsilon\right) \\
& =\operatorname{wind}\left(\Phi(m \cdot)^{-1}\left[U\left(R_{1}, \cdot\right)-U\left(R_{1}, \cdot+\frac{j}{m}\right)\right]\right) .
\end{aligned}
$$

Since $\Phi(m t)^{-1}\left[U(s, t)-U\left(s, t+\frac{j}{m}\right)\right]$ has no zeroes, we know that the winding is independent of $s$. We these observations with the definition of wind ${ }_{2}$ from the previous section to conclude that

$$
\begin{aligned}
i_{\infty}^{\Phi}(\tilde{u} ; z) & =\sum_{j=1}^{|m|-1} \operatorname{wind}\left(F_{j}\left(R_{2}, \cdot\right)\right)-\operatorname{wind}\left(F_{j}\left(R_{1}, \cdot\right)\right) \\
& =-\sum_{j=1}^{|m|-1} \operatorname{wind}\left(\Phi(m \cdot)^{-1}\left[U\left(R_{1}, \cdot\right)-U\left(R_{1}, \cdot+\frac{j}{m}\right)\right]\right)
\end{aligned}
$$




$$
=-\operatorname{wind}_{2}^{\Phi}(\tilde{u} ; z)
$$

as claimed.

As an immediate corollary of this computation and Lemma 3.14 we get that the asymptotic self-intersection number satisfies a number of properties. Note that (3-28) below is an improvement on [14, Lemma 6.7] and reduces to the result given there when $\operatorname{cov}\left(e_{1}(\tilde{u} ; z)\right)=1$.

Corollary 3.18 Let $[\tilde{u} ; z]=[\Sigma, j, z, \tilde{u}]$ be an embedded end, with asymptotic limit $\gamma^{m}$, and let $\Phi$ and $\Psi$ be unitary trivializations of $\gamma^{*} \xi^{\mathcal{H}}$. Then the relative asymptotic self-intersection number of the end $[\tilde{u} ; z]$ satisfies

$$
i_{\infty}^{\Phi}(\tilde{u} ; z)=i_{\infty}^{\Psi}(\tilde{u} ; z)-m(|m|-1) \operatorname{deg}\left(\Phi^{-1} \circ \Psi\right)
$$

If $[\tilde{u} ; z]$ is a pseudoholomorphic end, then:

- We have that

$$
i_{\infty}^{\Phi}(\tilde{u} ; z) \geq-(|m|-1) \operatorname{wind}_{\infty}^{\Phi}(\tilde{u} ; z)+\operatorname{cov}\left(e_{1}(\tilde{u} ; z)\right)-1
$$

and that

$$
i_{\infty}^{\Phi}(\tilde{u} ; z)=-(|m|-1) \operatorname{wind}_{\infty}^{\Phi}(\tilde{u} ; z)
$$

if and only if $\operatorname{cov}\left(e_{1}(\tilde{u} ; z)\right)=1$.

- In particular, we have that

$$
i_{\infty}^{\Phi}(\tilde{u} ; z) \geq-(|m|-1) \alpha^{\Phi}(\tilde{u} ; z)+\bar{\sigma}(\tilde{u} ; z)-1
$$

and that

$$
i_{\infty}^{\Phi}(\tilde{u} ; z)=-(|m|-1) \alpha^{\Phi}(\tilde{u} ; z)
$$

if and only if $d_{0}(\tilde{u} ; z)=0$ and $\bar{\sigma}(\tilde{u} ; z)=1$.

The lower bounds on $i_{\infty}^{\Phi}(\tilde{u} ; z)$ should be thought of as a sort of "positivity of intersections at infinity" for embedded pseudoholomorphic ends. Indeed, we have shown that the quantity

$$
i_{\infty}^{\Phi}(\tilde{u} ; z)+(|m|-1) \alpha^{\Phi}(\tilde{u} ; z)
$$

is independent of choice of trivialization and that

$$
\begin{aligned}
i_{\infty}^{\Phi}(\tilde{u} ; z)+(|m|-1) \alpha^{\Phi}(\tilde{u} ; z) & =\operatorname{wind}_{2}^{\Phi}(\tilde{u} ; z)+(|m|-1) \alpha^{\Phi}(\tilde{u} ; z) \\
& =\Delta_{1}(\tilde{u} ; z)+\Delta_{2}(\tilde{u} ; z)+[\bar{\sigma}(\tilde{u} ; z)-1]
\end{aligned}
$$


where each of the three terms on the right hand side of this equation are nonnegative quantities when $[\tilde{u} ; z]$ is pseudoholomorphic. We will define the asymptotic selfintersection index $\delta_{\infty}(\tilde{u} ; z)$ of the end $[\tilde{u} ; z]$ to be

$$
\begin{aligned}
\delta_{\infty}(\tilde{u} ; z) & :=\frac{1}{2}\left[i_{\infty}^{\Phi}(\tilde{u} ; z)+(|m|-1) \alpha^{\Phi}(\tilde{u} ; z)-\bar{\sigma}(\tilde{u} ; z)+1\right] \\
& =\frac{1}{2} \Delta_{t o t}(\tilde{u} ; z) .
\end{aligned}
$$

We note that for pseudoholomorphic ends the asymptotic self-intersection index takes values in the nonnegative integers as a result of Lemma 3.14 and Lemma 3.17. The asymptotic self-intersection index can be thought of as a measure of the self-tangency at infinity of an embedded pseudoholomorphic end. In any given trivialization, it counts one half the difference between the number of intersections the end has with a perturbed copy of itself and the minimum number that must occur for any embedded pseudoholomorphic end with the same asymptotic data.

We now move on to considering the asymptotic intersection properties of a pair of ends of curves which are asymptotic to coverings of the same simple periodic orbit with the same sign. Our assumptions will be that $(\Sigma, j, z, \tilde{u})$ and $\left(\Sigma^{\prime}, j^{\prime}, w, \tilde{v}\right)$ are nonintersecting (not necessarily pseudoholomorphic) end models in $\mathbb{R} \times M$. We further assume that $\tilde{u}$ is asymptotically cylindrical near $z$ over $\gamma^{m_{z}}$ and $\tilde{v}$ is asymptotically cylindrical near $w$ to $\gamma^{m_{w}}$ and that $m_{z}$ and $m_{w}$ have the same sign.

We assume that $(U, \psi)$ and $(V, \phi)$ are asymptotic representatives of $\tilde{u}$ and $\tilde{v}$ respectively so that we can write

$$
\begin{aligned}
\tilde{u} \circ \psi(s, t) & =\left(m_{z} \tau s, \exp _{\gamma^{m_{z}(t)}} U(s, t)\right) \\
\tilde{v} \circ \phi(s, t) & =\left(m_{w} \tau s, \exp _{\gamma^{m}(t)} V(s, t)\right)
\end{aligned}
$$

for $(s, t) \in[R, \infty) \times S^{1}$ for some $R \in \mathbb{R}$. If $\Phi$ is a trivialization of $\gamma^{*} \xi^{\mathcal{H}}$, we define a map $\tilde{v}_{w, \varepsilon, \Phi}:[R, \infty) \times S^{1} \rightarrow W$ as above by perturbing the map $\tilde{v}$ on a neighborhood of $w$ in a direction determined by $\Phi$. As above we have the following lemma.

Lemma 3.19 There exists an $\varepsilon_{0}>0$ so that the intersection number

$$
\operatorname{int}\left(\tilde{u}, \tilde{v}_{w, \varepsilon, \Phi}\right)
$$

is well-defined and independent of $\varepsilon \in\left(0, \varepsilon_{0}\right)$ and invariant under homotopies of unitary trivializations $\Phi$.

Proof Again denoting $C_{R}=[R, \infty) \times S^{1}$ and $I_{R}=[R, R+1] \times S^{1}$, it follows from the asymptotic behavior of $(\tilde{u} ; z)$ and $(\tilde{v} ; w)$ that there exists an $\varepsilon_{1}>0$ so that

$$
\tilde{u} \circ \phi\left(I_{R}\right) \cap \tilde{v}_{w, \varepsilon, \Phi}\left(C_{R}\right)=\varnothing \quad \text { and } \quad \tilde{u} \circ \phi\left(C_{R}\right) \cap \tilde{v}_{w, \varepsilon, \Phi}\left(I_{R}\right)=\varnothing
$$


for and $\varepsilon \in\left(0, \varepsilon_{1}\right)$ and any unitary trivialization $\Phi$ of $\gamma^{*} \xi^{\mathcal{H}}$. Moreover, the asymptotic behavior of $\tilde{u}$ and $\tilde{v}$ implies that there exists an $\varepsilon_{2}$ so that for any $\varepsilon \in\left(0, \varepsilon_{2}\right)$ we can find an $R(\varepsilon)$ so that $\tilde{u} \circ \phi\left(C_{R(\varepsilon)}\right) \cap \tilde{v}_{w, \varepsilon, \Phi}\left(C_{R}\right)=\varnothing$ and $\tilde{u} \circ \phi\left(C_{R}\right) \cap \tilde{v}_{w, \varepsilon, \Phi}\left(C_{R(\varepsilon)}\right)=\varnothing$ for any unitary $\Phi$. Therefore choosing $\varepsilon_{0}>0$ less than $\min \left\{\varepsilon_{1}, \varepsilon_{2}\right\}$, it follows that for any $\varepsilon \in\left(0, \varepsilon_{0}\right)$ we have

and

$$
\begin{aligned}
& \tilde{u} \circ \phi\left(I_{R} \cup C_{R(\varepsilon)}\right) \cap \tilde{v}_{w, \varepsilon, \Phi}\left(C_{R}\right)=\varnothing \\
& \tilde{u} \circ \phi\left(C_{R}\right) \cap \tilde{v}_{w, \varepsilon, \Phi}\left(I_{R} \cup C_{R(\varepsilon)}\right)=\varnothing
\end{aligned}
$$

for any unitary $\Phi$. Given this, the claims of the lemma follow from standard transversality and homotopy invariance arguments as in Lemma 3.16.

We use this lemma to define a local invariant of a pair of nonintersecting asymptotically cylindrical ends. Let $(\Sigma, j, z, \tilde{u})$ and $\left(\Sigma^{\prime}, j^{\prime}, w, \tilde{v}\right)$ still denote nonintersecting end models asymptotically cylindrical over $\gamma^{m_{z}}$ and $\gamma^{m_{w}}$ respectively, with $m_{z}$ and $m_{w}$ having the same sign. Let $O$ be some open neighborhood of $w$ and let $v_{\varepsilon}, \Phi$ still denote a perturbation of $\left.\tilde{v}\right|_{O}$ defined as above. The preceding lemma shows that the intersection number

$$
\operatorname{int}\left(\tilde{u}, \tilde{v}_{\varepsilon, \Phi}\right)
$$

is independent of sufficiently small $\varepsilon>0$ and depends only on the maps $\tilde{u}$ and $\tilde{v}$ and the homotopy class of the unitary trivialization $\Phi$. Using this observation, we define the relative asymptotic intersection number of the end models $(\Sigma, j, z, \tilde{u})$ and $\left(\Sigma^{\prime}, j^{\prime}, w, \tilde{v}\right)$ relative to $\Phi$ by

$$
i_{\infty}^{\Phi}([\tilde{u} ; z],[\tilde{v} ; w]):=\operatorname{int}\left(\left.\tilde{u}\right|_{O}, \tilde{v}_{\varepsilon, \Phi}\right)
$$

where $\varepsilon>0$ is chosen small enough so that the conclusions of the previous lemma apply.

As with the relative asymptotic self-intersection number, we can use Theorem 3.6 to compute the relative asymptotic intersection number in terms of the local windingrelated invariants studied in the previous section.

Lemma 3.20 Let $(\Sigma, j, z, \tilde{u})$ and $\left(\Sigma^{\prime}, j^{\prime}, w, \tilde{v}\right)$ be nonintersecting asymptotically cylindrical end models and assume that $(\Sigma, j, z, \tilde{u})$ and $\left(\Sigma^{\prime}, j^{\prime}, w, \tilde{v}\right)$ are asymptotically cylindrical over $\gamma^{m_{z}}$ and $\gamma^{m_{w}}$ respectively with $m_{z} m_{w}>0$. Then the relative asymptotic intersection number of $(\tilde{u} ; z)$ and $(\tilde{v} ; w)$ is given by

$$
i_{\infty}^{\Phi}([\tilde{u} ; z],[\tilde{v} ; w])=-\frac{1}{m_{z} m_{w}} \operatorname{wind}_{\mathrm{rel}}^{\Phi}\left(\left|m_{w}\right| \cdot[\tilde{u} ; z],\left|m_{z}\right| \cdot[\tilde{v} ; w]\right)
$$

where $\Phi$ is a unitary trivialization of $\gamma^{*} \xi^{\mathcal{H}}$ and $m \cdot$ is the operation of multiply covering an asymptotically cylindrical end. 
We remark that, as with the relative asymptotic self-intersection number, this lemma demonstrates that the relative asymptotic intersection number is an invariant of the ends $[\Sigma, j, z, \tilde{u}]$ and $\left[\Sigma^{\prime}, j^{\prime}, w, \tilde{v}\right]$ and does not depend on the choice of models representing these ends.

Proof We initially assume that the ends in question cover $\gamma$ with the same covering number, ie that $m_{z}=m_{w}$. In this case, an argument analogous to that in Lemma 3.17 shows that

$$
i_{\infty}^{\Phi}([\tilde{u} ; z],[\tilde{v} ; w])=-\operatorname{wind}_{\mathrm{rel}}^{\Phi}([\tilde{u} ; z],[\tilde{v} ; w]) .
$$

Moreover, it is an easy consequence of the definition of wind $_{\text {rel }}$ that

$$
\operatorname{wind}_{\mathrm{rel}}^{\Phi}(m \cdot[\tilde{u} ; z], m \cdot[\tilde{v} ; w])=m^{2} \operatorname{wind}_{\mathrm{rel}}^{\Phi}([\tilde{u} ; z],[\tilde{v} ; w])
$$

for any positive integer $m$ so this is equivalent to the formula given in the statement of the lemma.

In the case that $[\tilde{u} ; z]$ and $[\tilde{v} ; w]$ cover $\gamma$ with different covering numbers (ie $m_{z} \neq m_{w}$ ) then we can replace $[\tilde{u} ; z]$ and $[\tilde{v} ; w]$ with the multiply covered ends $\left|m_{w}\right| \cdot[\tilde{u} ; z]$ and $\left|m_{z}\right| \cdot[\tilde{v} ; w]$ which both have covering number $m_{z}\left|m_{w}\right|=\left|m_{z}\right| m_{w}$. Then the reasoning of the previous paragraph gives

$$
i_{\infty}^{\Phi}\left(\left|m_{2}\right| \cdot[\tilde{u} ; z],\left|m_{z}\right| \cdot[\tilde{v} ; w]\right)=-\operatorname{wind}_{\mathrm{rel}}^{\Phi}\left(\left|m_{2}\right| \cdot[\tilde{u} ; z],\left|m_{w}\right| \cdot[\tilde{v} ; w]\right) .
$$

Moreover, it is an easy consequence of the definition of the relative asymptotic intersection number that

$$
i_{\infty}^{\Phi}\left(k_{1} \cdot[\tilde{u} ; z], k_{2} \cdot[\tilde{v} ; w]\right)=k_{1} k_{2} i_{\infty}^{\Phi}([\tilde{u} ; z],[\tilde{v} ; w])
$$

for any positive integers $k_{1}$ and $k_{2}$. The claim of the lemma follows immediately from these two equations.

This lemma used with the basic results about the total relative winding yields the following corollary. Note that (3-30) below is analogous to [14, Lemma 6.9].

Corollary 3.21 Let $[\tilde{u} ; z]$ and $[\tilde{v} ; w]$ be nonintersecting ends asymptotically cylindrical over $\gamma^{m_{z}}$ and $\gamma^{m_{w}}$ respectively and let $\Phi$ and $\Psi$ be unitary trivializations of $\gamma^{*} \xi^{\mathcal{H}}$. Then the relative asymptotic intersection number satisfies

$$
i_{\infty}^{\Phi}([\tilde{u} ; z],[\tilde{v} ; w])=i_{\infty}^{\Phi}([\tilde{v} ; w],[\tilde{u} ; z])
$$

and

$$
i_{\infty}^{\Phi}([\tilde{u} ; z],[\tilde{v} ; w])=i_{\infty}^{\Psi}([\tilde{u} ; z],[\tilde{v} ; w])-m_{z}\left|m_{w}\right| \operatorname{deg}(\Phi \circ \Psi)
$$


If $[\tilde{u} ; z]$ and $[\tilde{v} ; w]$ are pseudoholomorphic, then

$$
\begin{aligned}
& i_{\infty}^{\Phi}([\tilde{u} ; z],[\tilde{v} ; w]) \geq-m_{z} m_{w} \max \left\{\frac{\operatorname{wind}_{\infty}^{\Phi}(\tilde{u} ; z)}{\left|m_{z}\right|}, \frac{\operatorname{wind}_{\infty}^{\Phi}(\tilde{v} ; w)}{\left|m_{w}\right|}\right\} \\
& i_{\infty}^{\Phi}([\tilde{u} ; z],[\tilde{v} ; w]) \geq-m_{z} m_{w} \max \left\{\frac{\alpha^{\Phi}\left(\gamma^{m_{z}}\right)}{\left|m_{z}\right|}, \frac{\alpha^{\Phi}\left(\gamma^{m_{w}}\right)}{\left|m_{w}\right|}\right\} .
\end{aligned}
$$

Proof The first two statements follow directly from Lemma 3.20 and Lemma 3.15. The inequality (3-30) follows from these same Lemmas and the additional elementary fact that

$$
\operatorname{wind}_{\infty}^{\Phi}(m \cdot[\tilde{u} ; z])=m \operatorname{wind}_{\infty}^{\Phi}(\tilde{u} ; z)
$$

for any pseudoholomorphic end $[\tilde{u} ; z]$ and any positive integer $m$. Finally, the inequality (3-31) follows from (3-30) and that

$$
\operatorname{wind}_{\infty}^{\Phi}(\tilde{u} ; z) \leq \alpha^{\Phi}(\tilde{u} ; z)
$$

for pseudoholomorphic ends, as observed in Lemma 3.13.

In analogy to the the case of a single end, we define the asymptotic intersection index, $\delta_{\infty}([\tilde{u} ; z],[\tilde{v} ; w])$, of the pair of ends $[\tilde{u} ; z]$ and $[\tilde{v} ; w]$, satisfying the assumptions of the previous lemmas by

$$
\delta_{\infty}([\tilde{u} ; z],[\tilde{v} ; w]):=i_{\infty}^{\Phi}([\tilde{u} ; z],[\tilde{v} ; w])+m_{z} m_{w} \max \left\{\frac{\alpha^{\Phi}\left(\gamma^{m_{z}}\right)}{\left|m_{z}\right|}, \frac{\alpha^{\Phi}\left(\gamma^{m_{w}}\right)}{\left|m_{w}\right|}\right\}
$$

and we note that when $[\tilde{u} ; z]$ and $[\tilde{v} ; w]$ are pseudoholomorphic, this quantity is nonnegative as a result of the preceding corollary. The asymptotic intersection index can be thought of as a measure of the tangency at infinity of the two pseudoholomorphic ends. It counts the difference between the number of intersections that appear when one end is perturbed and the minimum number that must appear for any two nonintersecting pseudoholomorphic ends with the given asymptotic data.

\section{Global intersection theory}

\subsection{The generalized intersection number and positivity of intersections}

4.1.1 Relative intersection number In this section we introduce the relative intersection number and establish its basic properties. We consider two smooth asymptotically cylindrical maps $(\Sigma, j, \Gamma, \tilde{u}),\left(\Sigma^{\prime}, j^{\prime}, \Gamma^{\prime}, \tilde{v}\right) \in C^{\infty}\left(W, \mathcal{H}^{+}, \mathcal{H}^{-}\right)$where $W$ is a $4-$ manifold with Hamiltonian structured cylindrical ends. Given a trivialization $\Phi$ of the stable Hamiltonian hyperplane field along the periodic orbits, we define the relative 
intersection number $i^{\Phi}(\tilde{u}, \tilde{v})$ of $\tilde{u}$ and $\tilde{v}$ relative to $\Phi$ by perturbing $\tilde{v}$ near its punctures in a direction determined by $\Phi$ and computing the algebraic intersection number of $\tilde{u}$ with the perturbation of $\tilde{v}$. More precisely, given a puncture $w \in \Gamma^{\prime}$ of $\tilde{v}$, we can find a neighborhood $U$ of $w$ which gets mapped entirely within one of the cylindrical ends and so that

$$
\tilde{v}(\phi(s, t))=\left(m s, \exp _{\gamma^{m}(t)} h(s, t)\right) \in \mathbb{R}^{ \pm} \times M^{ \pm}
$$

for some simple periodic orbit $\gamma$ and $m \in \mathbb{Z} \backslash\{0\}$, where $\phi:[R, \infty) \times S^{1} \rightarrow U \backslash\{w\}$ is a diffeomorphism. Choosing some smooth cutoff function $\beta$ supported in $U$ and equal to 1 in a neighborhood of $w$, we define the perturbed end $\tilde{v}_{\Phi, \varepsilon}^{U}: U \backslash\{w\} \rightarrow \mathbb{R}^{ \pm} \times M^{ \pm}$ by

$$
\tilde{v}_{\Phi, \varepsilon}^{U}=\left(m s, \exp _{\gamma^{m}(t)}\left[h(s, t)+\beta(\phi(s, t)) \Phi_{\gamma}(m t) \varepsilon\right]\right) \in \mathbb{R}^{ \pm} \times M^{ \pm}
$$

where $\Phi_{\gamma}: S^{1} \times \mathbb{C} \rightarrow \gamma^{*} \xi^{\mathcal{H}^{ \pm}}$is a unitary trivialization of $\gamma^{*} \xi^{\mathcal{H}^{ \pm}}$and $\varepsilon \in \mathbb{R}^{+} \in \mathbb{C}$ is a small, positive real number. We define the map $\tilde{v}_{\Phi, \varepsilon}: \Sigma^{\prime} \backslash \Gamma^{\prime} \rightarrow W$ by replacing $\tilde{v}$ in a neighborhood of each puncture with a perturbation as in (4-1). It then follows from standard arguments that there exists an $\varepsilon_{0}>0$ so that for $\varepsilon \in\left(0, \varepsilon_{0}\right)$ the algebraic intersection number

$$
\operatorname{int}\left(\tilde{u}, \tilde{v}_{\Phi, \varepsilon}\right)
$$

is independent of all choices made except for that of the homotopy class of trivialization $\Phi$. We thus define the relative intersection number $i^{\Phi}(\tilde{u}, \tilde{v})$ of $\tilde{u}$ and $\tilde{v}$ relative to $\Phi$ by

$$
i^{\Phi}(\tilde{u}, \tilde{v}):=\operatorname{int}\left(\tilde{u}, \tilde{v}_{\Phi, \varepsilon}\right)
$$

for some sufficiently small $\varepsilon>0$.

We summarize important properties of the relative intersection number in the following proposition.

Proposition 4.1 Let $\tilde{u}$ and $\tilde{v}$ satisfy the assumptions of the previous paragraph and let $\Phi$ be a trivialization of $\xi^{\mathcal{H}^{ \pm}}$along the asymptotic limits of $\tilde{u}$ and $\tilde{v}$. Moreover, assume that at $z \in \Gamma, \tilde{u}$ is asymptotic to $\gamma_{z}^{m_{z}}$ and at $w \in \Gamma^{\prime}, \tilde{v}$ is asymptotic to $\gamma_{w}^{m_{w}}$. Then the relative intersection number $i^{\Phi}(\tilde{u}, \tilde{v})$ satisfies:

(1) $i^{\Phi}(\tilde{u}, \tilde{v})$ depends only on the homotopy classes of $\Phi, \tilde{u}$ and $\tilde{v}$

(2) $i^{\Phi}(\tilde{u}, \tilde{v})=i^{\Phi}(\tilde{v}, \tilde{u})$

(3) If $\Psi$ is another trivialization of $\xi^{\mathcal{H}^{ \pm}}$along the periodic orbit set of the ends, then

$$
i^{\Phi}(\tilde{u}, \tilde{v})=i^{\Psi}(\tilde{u}, \tilde{v})+\sum_{\substack{(z, w) \in \Gamma_{+} \times \Gamma_{+}^{\prime} \\ \gamma_{z}=\gamma_{w}}}-m_{z} m_{w} \operatorname{deg}\left(\Phi_{\gamma_{z}}^{-1} \circ \Psi_{\gamma_{w}}\right)
$$




$$
+\sum_{\substack{(z, w) \in \Gamma_{-} \times \Gamma_{-}^{\prime} \\ \gamma_{z}=\gamma_{w}}} m_{z} m_{w} \operatorname{deg}\left(\Phi_{\gamma_{z}}^{-1} \circ \Psi_{\gamma_{w}}\right)
$$

Proof Part (1) follows from standard transversality and homotopy-invariance arguments and we omit the straightforward details.

To see that part (2) is true, it suffices construct homotopies $\tilde{u}_{\tau}$ and $\tilde{v}_{\tau}$, so that

$$
\begin{aligned}
\tilde{u}_{0} & =\tilde{u} \\
\tilde{u}_{1} & =\tilde{u}_{\Phi, \varepsilon} \\
\tilde{v}_{0} & =\tilde{v}_{\Phi, \varepsilon} \\
\tilde{v}_{1} & =\tilde{v}
\end{aligned}
$$

and so that there exist neighborhoods $U$ of $\Gamma$ and $V$ of $\Gamma^{\prime}$ so that

and

$$
\begin{aligned}
& \tilde{u}_{\tau}(U \backslash \Gamma) \cap \tilde{v}_{\tau}\left(\Sigma^{\prime} \backslash \Gamma^{\prime}\right)=\varnothing \\
& \tilde{u}_{\tau}(\Sigma \backslash \Gamma) \cap \tilde{v}_{\tau}\left(V \backslash \Gamma^{\prime}\right)=\varnothing .
\end{aligned}
$$

for all $\tau \in[0,1]$. This again is straightforward and we omit the details.

Finally (3), follows from an argument analogous to that in [14, Lemma 2.5 (b)] and we refer the reader there for more detail.

For pseudoholomorphic maps, it follows from Corollary 3.10 that the relative intersection number can be computed by summing local intersection numbers and asymptotic intersection numbers as defined in the preceding section. Before stating the result, we establish some notation and terminology. Consider pseudoholomorphic curves $[\Sigma, j, \Gamma, \tilde{u}],\left[\Sigma^{\prime}, j^{\prime}, \Gamma^{\prime}, \tilde{v}\right] \in \mathcal{M}\left(W, \bar{J}, \mathcal{H}^{+}, \mathcal{H}^{-}\right)$. A small neighborhood of a puncture $z \in \Gamma$ determines a pseudoholomorphic end (as defined in Section 3.1.2) which we will abbreviate $[\tilde{u} ; z]$. Thus if $[\tilde{u} ; z]$ and $[\tilde{v} ; w]$ are nonintersecting and asymptotic to a covering of the same orbit with the same sign, we can define the relative asymptotic intersection number

$$
i_{\infty}^{\Phi}([\tilde{u} ; z],[\tilde{v} ; w])
$$

as in the previous section. Otherwise, we define

$$
i_{\infty}^{\Phi}([\tilde{u} ; z],[\tilde{v} ; w])=0
$$

and we define the total relative asymptotic intersection number $i_{\infty}^{\Phi}(\tilde{u}, \tilde{v})$ of $[\Sigma, j, \Gamma, \tilde{u}]$ and $\left[\Sigma^{\prime}, j^{\prime}, \Gamma^{\prime}, \tilde{v}\right]$ by

$$
i_{\infty}^{\Phi}(\tilde{u}, \tilde{v}):=\sum_{(z, w) \in \Gamma \times \Gamma^{\prime}} i_{\infty}^{\Phi}([\tilde{u} ; z],[\tilde{v} ; w]) .
$$


Next, we say that $[\Sigma, j, \Gamma, \tilde{u}]$ and $\left[\Sigma^{\prime}, j^{\prime}, \Gamma^{\prime}, \tilde{v}\right] \in \mathcal{M}\left(W, \bar{J}, \mathcal{H}^{+}, \mathcal{H}^{-}\right)$have no common components if $\tilde{u}^{-1}\left(\tilde{v}\left(\Sigma^{\prime} \backslash \Gamma^{\prime}\right)\right)$ does not contain an open set. In this case, it follows from Corollary 3.10 that $\tilde{u}$ and $\tilde{v}$ intersect in a finite number of points. We can thus define the algebraic intersection number of $\tilde{u}$ and $\tilde{v}$ by summing local intersection numbers. We now state the result giving the relative intersection number of two pseudoholomorphic curves. This result is analogous to [14, Lemma 8.5] in the case that the $S_{1}$ and $S_{2}$ considered there are pseudoholomorphic. The result follows immediately from local intersection properties of pseudoholomorphic curves, Corollary 3.10 and the definitions of the terms involved, and we omit the straightforward proof.

Theorem 4.2 Let $[\Sigma, j, \Gamma, \tilde{u}],\left[\Sigma^{\prime}, j^{\prime}, \Gamma^{\prime}, \tilde{v}\right] \in \mathcal{M}\left(W, \bar{J}, \mathcal{H}^{+}, \mathcal{H}^{-}\right)$be a pair of pseudoholomorphic curves having no common components. Then

$$
i^{\Phi}(\tilde{u}, \tilde{v})=\operatorname{int}(\tilde{u}, \tilde{v})+i_{\infty}^{\Phi}(\tilde{u}, \tilde{v}) .
$$

4.1.2 The generalized intersection number We now define the generalized intersection number of two asymptotically cylindrical maps. This quantity will be an integer-valued symmetric product on the space of (homotopy classes of) smooth asymptotically cylindrical maps, which for pseudoholomorphic curves will bound the algebraic intersection number from above.

Motivated by the bounds on relative asymptotic intersection numbers given by Corollary 3.21 above, we define a homotopy-invariant product on asymptotically cylindrical maps by adding a term to the relative intersection pairing that balances the trivialization dependence. Consider two smooth asymptotically cylindrical maps $(\Sigma, j, \Gamma, \tilde{u})$ and $\left(\Sigma^{\prime}, j^{\prime}, \Gamma^{\prime}, \tilde{v}\right) \in C^{\infty}\left(W, \mathcal{H}^{+}, \mathcal{H}^{-}\right)$with $W$ as usual denoting a $4-$ dimensional manifold with Hamiltonian structured ends. Assume that at $z \in \Gamma_{ \pm}$that $\tilde{u}$ is asymptotic to $\gamma_{z}^{m_{z}}$ and at $w \in \Gamma_{ \pm}^{\prime}$ that $\tilde{v}$ is asymptotic to over $\gamma_{w}^{m_{w}}$. Given a trivialization of $\xi^{\mathcal{H}^{ \pm}}$along the asymptotic limits of $\tilde{u}$ and $\tilde{v}$, define a quantity $\Omega^{\Phi}(\tilde{u}, \tilde{v})$ by $^{6}$

$$
\begin{aligned}
\Omega^{\Phi}(\tilde{u}, \tilde{v}):= & \sum_{\substack{(z, w) \in \Gamma_{+} \times \Gamma_{+}^{\prime} \\
\gamma_{z}=\gamma_{w}}} m_{z} m_{w} \max \left\{\frac{\alpha^{\Phi}\left(\gamma_{z}^{m_{z}}\right)}{m_{z}}, \frac{\alpha^{\Phi}\left(\gamma_{w}^{m w}\right)}{m_{w}}\right\} \\
& +\sum_{\substack{(z, w) \in \Gamma_{-} \times \Gamma_{-}^{\prime} \\
\gamma_{z}=\gamma_{w}}} m_{z} m_{w} \max \left\{\frac{\alpha^{\Phi}\left(\gamma_{z}^{m_{z}}\right)}{-m_{z}}, \frac{\alpha^{\Phi}\left(\gamma_{w}^{m w}\right)}{-m_{w}}\right\} .
\end{aligned}
$$

${ }^{6}$ Note that this is the same quantity appearing in the sum on the right hand side of formula (2-3) from the introduction, except here we use that $\alpha^{\Phi}\left(\gamma^{k}\right)=\left\lfloor\mu^{\Phi}\left(\gamma^{k}\right) / 2\right\rfloor$ (see (3-4)) and we explicitly separate the parts of the sum coming respectively from positive punctures and negative punctures. 
Note that this quantity depends only on the asymptotic data associated to the two maps and not on the maps themselves. We then define the generalized intersection number of two asymptotically cylindrical maps by

$$
[\tilde{u}] *[\tilde{v}]=i^{\Phi}(\tilde{u}, \tilde{v})+\Omega^{\Phi}(\tilde{u}, \tilde{v}) .
$$

We note that as an immediate consequence of the change of trivialization formulas for $i^{\Phi}$ and $\alpha^{\Phi}$ from Proposition 4.1 and Lemma 3.4 the generalized intersection number does not depend on the choice of trivialization.

We now prove Theorem 2.1 which gives the basic properties of the generalized intersection number. We restate the result here for the convenience of the reader.

Proposition 4.3 (Properties of the generalized intersection number) Let $W, W_{1}$ and $W_{2}$ be 4-manifolds with Hamiltonian structured cylindrical ends and assume we can form the concatenation $W_{1} \odot W_{2}$. Then:

(1) If $(\Sigma, j, \Gamma, \tilde{u})$ and $\left(\Sigma^{\prime}, j^{\prime}, \Gamma^{\prime}, \tilde{v}\right) \in C^{\infty}\left(W, \mathcal{H}^{+}, \mathcal{H}^{-}\right)$are asymptotically cylindrical maps then the generalized intersection number $[\tilde{u}] *[\tilde{v}]$ depends only on the homotopy classes of $\tilde{u}$ and $\tilde{v}$.

(2) For any $(\Sigma, j, \Gamma, \tilde{u})$ and $\left(\Sigma^{\prime}, j^{\prime}, \Gamma^{\prime}, \tilde{v}\right) \in C^{\infty}\left(W, \mathcal{H}^{+}, \mathcal{H}^{-}\right)$

$$
[\tilde{u}] *[\tilde{v}]=[\tilde{v}] *[\tilde{u}] .
$$

(3) If $(\Sigma, j, \Gamma, \tilde{u}),\left(\Sigma^{\prime}, j^{\prime}, \Gamma^{\prime}, \tilde{v}\right),\left(\Sigma^{\prime \prime}, j^{\prime \prime}, \Gamma^{\prime \prime}, \tilde{w}\right) \in C^{\infty}\left(W, \mathcal{H}^{+}, \mathcal{H}^{-}\right)$then

$$
[\tilde{u}+\tilde{v}] *[\tilde{w}]=[\tilde{u}] *[\tilde{w}]+[\tilde{v}] *[\tilde{w}]
$$

where "+" on the left hand side denotes the disjoint union of the maps $\tilde{u}$ and $\tilde{v}$.

(4) If $u_{1} \odot u_{2}$ and $v_{1} \odot v_{2}$ are asymptotically cylindrical buildings in $W_{1} \odot W_{2}$ then

$$
\left[u_{1} \odot u_{2}\right] *\left[v_{1} \odot v_{2}\right] \geq\left[u_{1}\right] *\left[v_{1}\right]+\left[u_{2}\right] *\left[v_{2}\right] .
$$

Moreover, strict inequality occurs if and only if there is a periodic orbit $\gamma$ so that $\tilde{u}_{1}$ has a negative puncture asymptotic to $\gamma^{m}, \tilde{v}_{1}$ has a negative puncture asymptotic to $\gamma^{n}$ and both $\gamma^{m}$ and $\gamma^{n}$ are odd orbits.

Proof The first claim follows immediately from the fact that $i_{\infty}^{\Phi}(\tilde{u}, \tilde{v})$ and $\Omega^{\Phi}(\tilde{u}, \tilde{v})$ are determined entirely by the homotopy classes of $\tilde{u}, \tilde{v}$ and $\Phi$. Since their sum does not depend on a choice of trivialization, the generalized intersection number only depends on the homotopy class of $\tilde{u}$ and $\tilde{v}$ in $C^{\infty}\left(W, \mathcal{H}^{+}, \mathcal{H}^{-}\right)$.

The second claim follows immediately from the symmetry of the relative intersection number and the quantity $\Omega^{\Phi}(\tilde{u}, \tilde{v})$ and the third claim follows immediately from the definition and basic properties of intersection numbers. 
Before proving the final claim, we first establish some notation. We first observe that, since we assume that the concatenation $u_{1} \odot u_{2}$ is defined, the asymptotic data at the negative punctures of $u_{1}$ must correspond with and match the asymptotic data at the positive punctures of $u_{2}$. We will let $\Gamma_{1,-}=\left\{z_{i}^{-}\right\}$denote the set of negative punctures of $u_{1}$ and $\Gamma_{2,+}=\left\{z_{i}^{+}\right\}$denote the set of positive punctures of $u_{2}$. Moreover we assume that at $z_{i}^{+}, u_{2}$ is asymptotic to the periodic orbit $\gamma_{i}^{m_{i}}$, while at $z_{i}^{-}, u_{1}$ is asymptotic to the periodic orbit $\gamma_{i}^{-m_{i}}$. Similarly, let $\Gamma_{1,-}^{\prime}=\left\{w_{j}^{-}\right\}\left(\Gamma_{2,+}^{\prime}=\left\{w_{j}^{+}\right\}\right)$ be the set of negative (positive) punctures of $v_{1}\left(v_{2}\right)$ and assume that at $w_{j}^{+}\left(w_{j}^{-}\right)$that $v_{2}\left(v_{1}\right)$ is asymptotic to the periodic orbit $\hat{\gamma}_{j}^{n_{j}}\left(\hat{\gamma}_{j}^{-n_{j}}\right)$.

With this notation set, it follows from the definition of the generalized intersection number that

$$
\text { (4-4) }=\sum_{\substack{i, j \\ \gamma_{i}=\hat{\gamma}_{j}}}-m_{i} n_{j}\left(\max \left\{\frac{\alpha^{\Phi}\left(\gamma_{i}^{m_{i}}\right)}{m_{i}}, \frac{\alpha^{\Phi}\left(\hat{\gamma}_{j}^{n_{j}}\right)}{n_{j}}\right\}+\max \left\{\frac{\alpha^{\Phi}\left(\gamma_{i}^{-m_{i}}\right)}{m_{i}}, \frac{\alpha^{\Phi}\left(\hat{\gamma}_{j}^{-n_{j}}\right)}{n_{j}}\right\}\right)
$$

Consider then the expression

$$
\Delta(\gamma, m, n):=-m n\left(\max \left\{\frac{\alpha^{\Phi}\left(\gamma^{m}\right)}{m}, \frac{\alpha^{\Phi}\left(\gamma^{n}\right)}{n}\right\}+\max \left\{\frac{\alpha^{\Phi}\left(\gamma^{-m}\right)}{m}, \frac{\alpha^{\Phi}\left(\gamma^{-n}\right)}{n}\right\}\right)
$$

with $m$ and $n$ positive integers. If $\gamma$ is an even orbit, then Lemma 3.3 implies that

$$
\begin{aligned}
\Delta(\gamma, m, n) & =-m n\left(\max \left\{\frac{m \alpha^{\Phi}(\gamma)}{m}, \frac{n \alpha^{\Phi}(\gamma)}{n}\right\}+\max \left\{\frac{-m \alpha^{\Phi}(\gamma)}{m}, \frac{-n \alpha^{\Phi}(\gamma)}{n}\right\}\right) \\
& =0 .
\end{aligned}
$$

If $\gamma$ is odd and hyperbolic, we get

$$
\begin{aligned}
\Delta(\gamma, m, n)= & m n\left(\max \left\{\frac{m \alpha^{\Phi}(\gamma)+\frac{1}{2}(m-p(m))}{m}, \frac{n \alpha^{\Phi}(\gamma)+\frac{1}{2}(n-p(n))}{n}\right\}\right. \\
& \left.+\max \left\{\frac{-m \alpha^{\Phi}(\gamma)+\frac{1}{2}(-m-p(-m))}{m}, \frac{-n \alpha^{\Phi}(\gamma)+\frac{1}{2}(-n-p(-n))}{n}\right\}\right) \\
= & -m n\left(\max \left\{-\frac{p(m)}{m},-\frac{p(n)}{n}\right\}\right) \\
= & \min \{n p(m), m p(n)\} \geq 0,
\end{aligned}
$$


and we get a strict inequality here if and only if $m$ and $n$ are both odd. Finally, if $\gamma$ is elliptic, we get that there is an irrational $\theta$ so that

$$
\begin{aligned}
\Delta(\gamma, m, n) & =-m n\left(\max \left\{\frac{\lfloor m \theta\rfloor}{m}, \frac{\lfloor n \theta\rfloor}{n}\right\}+\max \left\{\frac{\lfloor-m \theta\rfloor}{m}, \frac{\lfloor-n \theta\rfloor}{n}\right\}\right) \\
& =\min \{-n\lfloor m \theta\rfloor,-m\lfloor n \theta\rfloor\}+\min \{-n\lfloor-m \theta\rfloor,-m\lfloor-n \theta\rfloor\} \\
& \geq-\lfloor n m \theta\rfloor-\lfloor-n m \theta\rfloor \\
& =\lceil n m \theta\rceil-\lfloor n m \theta\rfloor=1 .
\end{aligned}
$$

Thus for each pair $(i, j)$ with $\gamma_{i}=\hat{\gamma}_{j}$ and $\gamma_{i}^{m_{i}}$ and $\hat{\gamma}_{j}^{n_{j}}$ both having odd ConleyZehnder index, we get a positive term in the sum (4-4), which proves our claim.

We next prove Theorem 2.2 which is one of the main motivations for defining the generalized intersection number as we have. It says that for a pair of asymptotically cylindrical pseudoholomorphic curves with no common components, the generalized intersection number is equal to the algebraic intersection number plus the total measure of tangency at infinity between the two curves. Since the generalized intersection number is a homotopy invariant quantity, while the algebraic intersection number in general is not, this result demonstrates the utility of this quantity in situations where one is hoping to obtain topological control over the total count of intersections between two curves.

Before restating and proving the theorem we establish some notation. As previously noted, each puncture $z \in \Gamma$ of an asymptotically cylindrical pseudoholomorphic curve $[\Sigma, j, \Gamma, u] \in \mathcal{M}\left(W, \bar{J}, \mathcal{H}^{+}, \mathcal{H}^{-}\right)$determines a pseudoholomorphic end $[u ; z]$ (as defined in Section 3.1.2) in one of the ends of $W$. Given a second curve $\left[\Sigma^{\prime}, j^{\prime}, \Gamma^{\prime}, v\right]$ having no common components with $\tilde{u}$ and so that the ends $[u ; z]$ and $[v ; w]$ are asymptotic to a covering of the same orbit with the same sign, we can define the asymptotic intersection index

$$
\delta_{\infty}([u ; z],[v ; w])
$$

as before in (3-32). Otherwise, we define

$$
\delta_{\infty}([u ; z],[v ; w])=0,
$$

and we then define the total asymptotic intersection index $\delta_{\infty}(u, v)$ of $[\Sigma, j, \Gamma, u]$ and $\left[\Sigma^{\prime}, j^{\prime}, \Gamma^{\prime}, v\right]$ by

$$
\delta_{\infty}(u, v):=\sum_{(z, w) \in \Gamma \times \Gamma^{\prime}} \delta_{\infty}([u ; z],[v ; w]) .
$$

We now restate and prove Theorem 2.2. 
Theorem 4.4 (Positivity of the generalized intersection number) Let $\left(W^{4}, \bar{J}\right)$ be an almost complex cobordism with cylindrical ends $\left(\mathbb{R}^{ \pm} \times M^{ \pm}, J^{ \pm}, \mathcal{H}^{ \pm}\right)$and let $[\Sigma, j, \Gamma, u],\left[\Sigma^{\prime}, j^{\prime}, \Gamma^{\prime}, v\right] \in \mathcal{M}\left(W, \bar{J}, \mathcal{H}^{+}, \mathcal{H}^{-}\right)$be pseudoholomorphic curves in $W$ with no common components. Then

$$
[u] *[v]=\operatorname{int}(u, v)+\delta_{\infty}(u, v) .
$$

In particular

$$
[u] *[v] \geq \operatorname{int}(u, v) \geq 0,
$$

and

$$
[u] *[v]=0
$$

if and only if $u$ and $v$ do not intersect and the total asymptotic intersection number vanishes, ie $\delta_{\infty}(u, v)=0$.

Proof The equation (4-5) follows immediately from adding $\Omega^{\Phi}(u, v)$ to both sides of (4-2) and then applying the definition (3-32) of the asymptotic intersection index.

The final two claims are direct consequences of (4-5), local positivity of intersections for pseudoholomorphic curves and the nonnegativity of the asymptotic intersection index from (3-31) and (3-32).

\subsection{The adjunction formula}

4.2.1 Relative Chern numbers and the total Conley-Zehnder index In order to state the appropriate generalization of (1-1), we will need to introduce a relative version of the first Chern number of a complex vector bundle over a punctured Riemann surface. This will then be used to define the total Conley-Zehnder index of an asymptotically cylindrical map.

Let $(\Sigma, j)$ be a closed Riemann surface and let $\Gamma \subset \Sigma$ be a finite set. We will call an open neighborhood $U$ of $\Gamma$ disk-like if the closure $\bar{U}$ of $U$ is diffeomorphic to a disjoint union of closed disks and if each component of $U$ contains precisely one element of $\Gamma$. Let $E \rightarrow \Sigma \backslash \Gamma$ be a complex vector bundle. Since for any disk-like neighborhood $U$ of $\Gamma$ the set $U \backslash \Gamma$ has the homotopy type of a disjoint union of circles, the restriction $\left.E\right|_{U \backslash \Gamma}$ is trivial. Let $\Phi: U \backslash \Gamma \times \mathbb{C}^{n} \rightarrow E$ be a trivialization of $\left.E\right|_{U \backslash \Gamma}$. If $E$ is a line bundle, then we will define the first Chern number of $E$ relative to $\Phi$, denoted $c_{1}(E ; \Phi)$ or $c_{1}^{\Phi}(E)$, to be equal to the algebraic count of zeroes of a generic extension of the section $\Phi(z) 1$ of $\left.E\right|_{U \backslash \Gamma}$ to a section of $E$. If $\operatorname{dim}_{\mathbb{C}} E=n>1$, we define the first Chern number of $E$ relative to $\Phi$ by

$$
c_{1}(E ; \Phi)=c_{1}\left(\Lambda_{\mathbb{C}}^{n} E ; \Lambda_{\mathbb{C}}^{n} \Phi\right)
$$


where $\Lambda_{\mathbb{C}}^{n} E$ is the determinant line bundle of $E$ and $\Lambda_{\mathbb{C}}^{n} \Phi$ is the trivialization of $\left.\Lambda_{\mathbb{C}}^{n} E\right|_{U \backslash \Gamma}$ induced by $\Phi$. We can define an equivalence relation on the set of pairs $(U, \Phi)$ of disk-like neighborhoods of $\Gamma$ and trivializations $\Phi$ of $\left.E\right|_{U \backslash \Gamma}$ be defining $(U, \Phi) \sim(V, \Psi)$ provided there exists a disk-like neighborhood $U^{\prime} \subset U \cap V$ of $\Gamma$ so that $\left.\Phi\right|_{U^{\prime} \backslash \Gamma}$ is homotopic to $\left.\Psi\right|_{U^{\prime} \backslash \Gamma}$. The relative first Chern number $c_{1}(E ; \Phi)$ clearly only depends on the equivalence class of the pair $(U, \Phi)$.

We collect some useful properties of the relative first Chern number in the lemma below, but before stating the lemma we will establish some notation and terminology. Given a punctured Riemann surface $\Sigma \backslash \Gamma$, we will call a pair $(U, \psi)$ a holomorphic cylindrical coordinate system around $\Gamma$ if $U$ is a disk-like neighborhood of $\Gamma$ and $\psi$ is a biholomorphic map $\psi: \amalg_{i=1}^{\# \Gamma}\left([0, \infty) \times S^{1}\right)_{i} \rightarrow \bar{U} \backslash \Gamma$. Using the coordinate fields $\partial_{s}$ and $\partial_{t}$ to identify $T_{(s, t)}\left([0, \infty) \times S^{1}\right)$ with $\mathbb{C}$, we note that the derivative of $\psi$ is a map

$$
d \psi: T\left(\amalg_{i=1}^{\# \Gamma}\left([0, \infty) \times S^{1}\right)_{i}\right) \approx\left(\amalg_{i=1}^{\# \Gamma}\left([0, \infty) \times S^{1}\right)_{i}\right) \times\left.\mathbb{C} \rightarrow T \Sigma\right|_{U \backslash \Gamma}
$$

and therefore determines a trivialization of $(T \Sigma, j)$ over $U \backslash \Gamma$. If $E \rightarrow \Sigma \backslash \Gamma$ is a complex bundle, and $\Phi$ and $\Psi$ are trivializations of $\left.E\right|_{U \backslash \Gamma}$, then $\Phi^{-1} \circ \Psi$ determines a map $U \backslash \Gamma \rightarrow G L_{n}(\mathbb{C})$. If we denote $\psi_{i}=\left.\psi\right|_{\left([0, \infty) \times S^{1}\right)_{i}}$, then $\operatorname{det}\left(\left(\Phi^{-1} \circ \Psi\right)\left(\psi_{i}\left(s^{\prime}, \cdot\right)\right)\right)$ is an oriented loop in $\mathbb{C} \backslash\{0\}$ for any fixed $s^{\prime} \in \mathbb{R}^{+}$. We define $\operatorname{deg}\left(\Phi^{-1} \circ \Psi\right)$ by

$$
\operatorname{deg}\left(\Phi^{-1} \circ \Psi\right)=\sum_{i=1}^{\# \Gamma} \operatorname{wind}\left(\operatorname{det}\left(\left(\Phi^{-1} \circ \Psi\right)\left(\psi_{i}\left(s^{\prime}, \cdot\right)\right)\right)\right)
$$

and note that this definition does not depend on the choice of holomorphic cylindrical coordinates or on $s^{\prime}$.

Lemma 4.5 Let $(\Sigma, j)$ be a closed Riemann surface and let $\Gamma \subset \Sigma$ be a finite set. Let $E, E_{1}, E_{2} \rightarrow \Sigma \backslash \Gamma$ be complex vector bundles over $\Sigma \backslash \Gamma$ and let $U$ be a disk-like neighborhood of $\Gamma$. Then

- If $\Phi$ and $\Psi$ are each trivializations of $\left.E\right|_{U \backslash \Gamma}$ then

$$
c_{1}(E ; \Phi)=c_{1}(E ; \Psi)-\operatorname{deg}\left(\Phi^{-1} \circ \Psi\right) .
$$

- If $\Phi_{i}$ is a trivialization of $\left.E_{i}\right|_{U \backslash \Gamma}$ for $i \in\{1,2\}$, then

$$
c_{1}\left(E_{1} \oplus E_{2} ; \Phi_{1} \oplus \Phi_{2}\right)=c_{1}\left(E_{1} ; \Phi_{1}\right)+c_{1}\left(E_{2} ; \Phi_{2}\right)
$$

and

$$
c_{1}\left(E_{1} \otimes E_{2} ; \Phi_{1} \otimes \Phi_{2}\right)=c_{1}\left(E_{1} ; \Phi_{1}\right)+c_{1}\left(E_{2} ; \Phi_{2}\right)
$$


- If $(U, \psi)$ is a holomorphic cylindrical coordinate system, then

$$
c_{1}(T(\Sigma \backslash \Gamma) ; d \psi)=\chi(\Sigma \backslash \Gamma)=\chi(\Sigma)-\# \Gamma
$$

where $\chi(S)$ denotes the Euler characteristic of $S$.

Proof To see that (4-6) is true, we pick a holomorphic cylindrical coordinate system $(U, \psi)$ and choose a generic section $h$ of $\left.E\right|_{U \backslash \Gamma}$ which is equal to $\Lambda_{\mathbb{C}}^{n} \Phi(z) 1$ for $z \in \cup_{i} \psi_{i}\left([0,1] \times S^{1}\right)$ and equal to $\Lambda_{\mathbb{C}}^{n} \Psi(z) 1$ for $z \in \cup_{i} \psi_{i}\left([2, \infty) \times S^{1}\right)$. Extending $h$ to a generic section of $\Lambda_{\mathbb{C}}^{n} E$, the algebraic count of zeroes of $h$ in $\Sigma \backslash \Gamma$ is equal to $c_{1}(E ; \Psi)$ while the algebraic count of zeroes in $\Sigma \backslash U$ is equal to $c_{1}(E ; \Phi)$. Therefore, letting $m$ represent the algebraic count of zeroes of $h$ in the necks $\cup_{i} \psi_{i}\left([1,2] \times S^{1}\right)$, we must have

$$
c_{1}(E ; \Phi)=c_{1}(E ; \Psi)+m
$$

so it suffices to show $m=-\operatorname{deg}\left(\Phi^{-1} \circ \Psi\right)$. This indeed follows from

$$
\begin{aligned}
m= & \\
& \sum_{i=1}^{\# \Gamma} \operatorname{wind}\left(\left(\Lambda_{\mathbb{C}}^{n} \Phi\left(\psi_{i}(2, \cdot)\right)\right)^{-1} h\left(\psi_{i}(2, \cdot)\right)\right)-\operatorname{wind}\left(\left(\Lambda_{\mathbb{C}}^{n} \Phi\left(\psi_{i}(1, \cdot)\right)\right)^{-1} h\left(\psi_{i}(1, \cdot)\right)\right) \\
= & \sum_{i=1}^{\# \Gamma} \operatorname{wind}(1)-\operatorname{wind}\left(\left(\Lambda_{\mathbb{C}}^{n} \Phi\left(\psi_{i}(1, \cdot)\right)\right)^{-1}\left(\Lambda_{\mathbb{C}}^{n} \Psi\left(\psi_{i}(1, \cdot)\right)\right) 1\right) \\
= & \sum_{i=1}^{\# \Gamma}-\operatorname{wind}\left(\Lambda_{\mathbb{C}}^{n}\left(\Phi^{-1} \circ \Psi\right)\left(\psi_{i}(1, \cdot)\right) 1\right)=\sum_{i=1}^{\# \Gamma}-\operatorname{wind}\left(\operatorname{det}\left(\left(\Phi^{-1} \circ \Psi\right)\left(\psi_{i}(1, \cdot)\right)\right)\right) \\
= & -\operatorname{deg}\left(\Phi^{-1} \circ \Psi\right) .
\end{aligned}
$$

To prove (4-7) and (4-8), we first observe that there are natural isomorphisms

and

$$
\begin{gathered}
\Lambda_{\mathbb{C}}^{n_{1}+n_{2}}\left(E_{1} \oplus E_{2}\right) \rightarrow\left(\Lambda_{\mathbb{C}}^{n_{1}} E_{1}\right) \otimes\left(\Lambda_{\mathbb{C}}^{n_{2}} E_{2}\right) \\
\Lambda_{\mathbb{C}}^{n_{1} n_{2}}\left(E_{1} \otimes E_{2}\right) \rightarrow\left(\Lambda_{\mathbb{C}}^{n_{1}} E_{1}\right) \otimes\left(\Lambda_{\mathbb{C}}^{n_{2}} E_{2}\right)
\end{gathered}
$$

where $n_{i}=\operatorname{dim}_{\mathbb{C}} E_{i}$. Furthermore, the trivializations $\Lambda_{\mathbb{C}}^{n_{1}+n_{2}}\left(\Phi_{1} \oplus \Phi_{2}\right)$ of $\left.\Lambda_{\mathbb{C}}^{n_{1}+n_{2}}\left(E_{1} \oplus E_{2}\right)\right|_{U \backslash \Gamma}$ and $\Lambda_{\mathbb{C}}^{n_{1} n_{2}}\left(\Phi_{1} \otimes \Phi_{2}\right)$ of $\left.\Lambda_{\mathbb{C}}^{n_{1} n_{2}}\left(E_{1} \otimes E_{2}\right)\right|_{U \backslash \Gamma}$ both induce the trivialization $\left(\Lambda_{\mathbb{C}}^{n_{1}} \Phi_{1}\right) \otimes\left(\Lambda_{\mathbb{C}}^{n_{2}} \Phi_{2}\right)$ on $\left.\left(\Lambda_{\mathbb{C}}^{n_{1}} E_{1}\right) \otimes\left(\Lambda_{\mathbb{C}}^{n_{2}} E_{2}\right)\right|_{U \backslash \Gamma}$ under the given isomorphisms. Therefore both (4-7) and (4-8) are equivalent to the special case of (4-8) when $E_{1}$ and $E_{2}$ are line bundles. In this case, we let $h_{i}$ be a generic section of $E_{i}$ agreeing with $\Phi_{i} 1$ over $U \backslash \Gamma$, we assume that the zero loci of $h_{1}$ and $h_{2}$ are disjoint. Then $h_{1} \otimes h_{2}$ is a section of $E_{1} \otimes E_{2}$ agreeing with $\left(\Phi_{1} \otimes \Phi_{2}\right) 1$ over $U \backslash \Gamma$ and the 
algebraic count of zeroes of $h_{1} \otimes h_{2}$ is given by the sum of the algebraic counts of zeroes of $h_{1}$ and $h_{2}$.

Finally, to see that (4-9) holds, we note that if $(U, \psi)$ is holomorphic cylindrical coordinate system then the section $d \psi(z) 1$ of $U \backslash \Gamma$ extends to a smooth section of $U$ with a simple zero of positive index at each point of $\Gamma$. Any extension of this section to a generic section of $T \Sigma$ will have $\chi(\Sigma)$ zeroes counted with sign, of which $\chi(\Sigma)-\# \Gamma$ lie within $\Sigma \backslash \Gamma$.

Having defined the relative first Chern number, we now define the total Conley-Zehnder index. Let $(\Sigma, j, \Gamma, u) \in C^{\infty}\left(W, \mathcal{H}^{+}, \mathcal{H}^{-}\right)$be an asymptotically cylindrical map in a 4-manifold $W$ with Hamiltonian structured ends and assume that $W$ is equipped with a compatible almost complex structure $\bar{J}$. A choice of unitary trivialization of $\left(\xi^{\mathcal{H}^{ \pm}}, J^{ \pm}\right)$along each asymptotic limit of $u$ induces a complex trivialization of the pull-back bundle $\left(u^{*} T W, \bar{J}\right)$ near the punctures of $u$ since on the cylindrical ends we have

$$
(T W, \bar{J}) \approx\left(\mathbb{R} \oplus \mathbb{R} X_{\mathcal{H}^{ \pm}} \oplus \xi^{\mathcal{H}^{ \pm}}, \widetilde{J}^{ \pm}\right) \approx \mathbb{C} X_{\mathcal{H}^{ \pm}} \oplus\left(\xi^{\mathcal{H}^{ \pm}}, J^{ \pm}\right) .
$$

We will use $\Phi$ to denote both the chosen trivialization of $\left(\xi^{\mathcal{H}^{ \pm}}, J^{ \pm}\right)$along the asymptotic limits and the induced trivialization of $\left(u^{*} T W, \bar{J}\right)$ near the punctures. We then define the total Conley-Zehnder index of $\mu(u)$ of $u$ by

$$
\mu(u)=2 c_{1}^{\Phi}\left(u^{*} T W, u^{*} \bar{J}\right)+\sum_{z \in \Gamma} \mu^{\Phi}\left(\gamma_{z}^{m_{z}}\right) .
$$

where $u$ is assumed to be asymptotic to $\gamma_{z}^{m_{z}}$ at $z \in \Gamma$. We note that this quantity is independent of choice of trivialization as a result of Lemma 3.4 and Lemma 4.5, and depends only on the homotopy classes of $u$ and compatible $\bar{J}$. We further note that in applications, the 4-manifold $W$ is typically equipped with a symplectic form with which $\bar{J}$ must be compatible on all of $W$ (as opposed to being just compatible on the ends as we assume here). In this case, it is well known (see eg Proposition 5 and discussion following in Section 1.3 of [13]) that the space of such $\bar{J}$ is contractible, so the total Conley-Zehnder index would depend only on the homotopy class of the map $u$.

4.2.2 The adjunction formula In this section we prove Theorem 2.3 which is a generalization of (1-1) for punctured curves that is stated in terms of the generalized intersection number.

Before proving the result, we review some basic facts about the local intersection properties of pseudoholomorphic curves which we will need. Proofs of these facts can be found in McDuff [17] or Micallef and White [18]. Let $u:(\Sigma, j) \rightarrow\left(W^{4}, J\right)$ be a 
pseudoholomorphic map in an almost complex 4-manifold. Since $d u: T_{z} \Sigma \rightarrow T_{u(z)} W$ is complex linear, the derivative of $u$ is always either rank 2 or rank 0 . Define the singular set $S(u)$ of $u$ to be the set of points where the derivative of $u$ is zero, ie

$$
S(u):=\{z \in \Sigma \mid d u(z)=0\} .
$$

Each point $z \in S(u)$ has an open neighborhood $U$ so that either $\left.u\right|_{U \backslash\{z\}}$ is an embedding or $\left.u\right|_{U \backslash\{z\}}$ factors through an embedding via a holomorphic covering. Consequently, the singular set of a pseudoholomorphic map is an isolated subset of the domain. For an asymptotically cylindrical pseudoholomorphic map, this fact combined with the assumed convergence to an orbit cylinder at the punctures implies that the singular set is finite.

Given a point $z \in S(u)$, assume that $\left.u\right|_{U \backslash\{z\}}$ is an embedding for some open neighborhood $U$ of $z$. We can define a quantity $\delta(u ; z)$ in the following way: there exists a $C^{1}$-small perturbation $J^{\prime}$ of $J$ supported near $u(z)$ and $C^{1}$-small perturbation $u^{\prime}$ of $u$ supported in a neighborhood $U$ of $z$ so that $\left.u^{\prime}\right|_{U}$ is an immersed $J^{\prime}$-holomorphic map with precisely $\delta(u ; z)$ transverse double points. This quantity is independent of the choice of perturbation.

Now, let $v:\left(\Sigma^{\prime}, j^{\prime}\right) \rightarrow\left(W^{4}, J\right)$ be another pseudoholomorphic map and assume that $u(p)=v(q)$ for some $(p, q) \in \Sigma \times \Sigma^{\prime}$. Then there exist open neighborhoods $U$ of $p$ and $V$ of $q$ so that either

$$
V \subset v^{-1}(u(U))
$$

or

$$
u(U \backslash\{p\}) \cap v(V \backslash\{q\})=\varnothing .
$$

In the latter case, when $u$ and $v$ have an isolated intersection at $u(p)=v(q)$, we will denote the local intersection number of $u$ at $p$ with $v$ at $q$ by

$$
\delta((u ; p),(v ; q)) .
$$

The local intersection number $\delta((u ; p),(v ; q))$ is always greater than or equal to 1 and is equal to 1 if and only if $u$ is immersed at $p, v$ is immersed at $q$, and $u$ and $v$ intersect transversely.

Assume next that $u:(\Sigma, j) \rightarrow\left(W^{4}, J\right)$ is a simple map, that is that $u$ does not factor through a branched cover of Riemann surfaces. Define the double point set $D_{u} \subset \Sigma \times \Sigma \backslash \Delta \Sigma$ of $u$ by

$$
D(u)=\{(p, q) \mid u(p)=u(q), p \neq q\} .
$$

As observed in Corollary 3.11, the preceding discussion along with Corollary 3.8 and Corollary 3.9 imply that an asymptotically cylindrical curve has at most a finite number 
of double points. For such a map, we define the self-intersection index $\delta(u)$ of $u$ by

$$
\delta(u)=\sum_{z \in S(u)} \delta(u ; z)+\frac{1}{2} \sum_{(z, w) \in D(u)} \delta((u ; z),(u ; w)) .
$$

We note that this quantity is an integer since the second sum counts $(z, w)$ and $(w, z)$ separately. A pseudoholomorphic map $u$ is an embedding if and only if $\delta(u)=0$. Furthermore, $\delta(u)$ has the following significance: if $J^{\prime}$ is a compactly supported, perturbation of $J$ and $u^{\prime}$ is an immersed, $J^{\prime}$-holomorphic, compactly supported perturbation of $u$ having only transverse double points, then $u^{\prime}$ has precisely $\delta(u)$ double points, that is $\delta(u)=\frac{1}{2} \# D(u)$.

Now consider an almost complex cobordism of Hamiltonian structures $\left(W, \bar{J}, \mathcal{H}^{+}, \mathcal{H}^{-}\right)$ and let $[\Sigma, j, \Gamma, u] \in \mathcal{M}\left(W, \bar{J}, \mathcal{H}^{+}, \mathcal{H}^{-}\right)$be a simple pseudoholomorphic curve. We define the total asymptotic self-intersection index of $u$ by

$$
\delta_{\infty}(u)=\sum_{z \in \Gamma} \delta_{\infty}(u ; z)+\frac{1}{2} \sum_{\substack{(z, w) \in \Gamma \times \Gamma \\ z \neq w}} \delta_{\infty}([u ; z],[u ; w]),
$$

where $\delta_{\infty}(u ; z)$ is as defined in (3-29) and $\delta_{\infty}([u ; z],[u ; w])$ is as defined in (3-32). This quantity will be nonnegative integer-valued since the asymptotic intersection index $\delta_{\infty}([u ; z],[u ; w])$ is symmetric and each pair of distinct punctures is counted twice in the second sum.

Letting $[\Sigma, j, \Gamma, u] \in \mathcal{M}\left(W, \bar{J}, \mathcal{H}^{+}, \mathcal{H}^{-}\right)$still denote a simple curve, we now define the singularity index ${ }^{7} \operatorname{sing}(u)$ of $u$ by

$$
\operatorname{sing}(u):=\delta(u)+\delta_{\infty}(u) .
$$

The singularity index is nonnegative-integer-valued and equals zero for a given curve if and only if that curve is embedded and has total asymptotic self-intersection index equal to zero. Finally, recalling the definition (3-14) of the spectral covering number $\bar{\sigma}(u ; z)$ of a pseudoholomorphic end $[u ; z]$, we define the total spectral covering number of $u$ by

$$
\bar{\sigma}(u)=\sum_{z \in \Gamma} \bar{\sigma}(u ; z)
$$

The spectral covering number depends only on the asymptotic data of the map and not on the map itself.

We now prove Theorem 2.3 which we restate here for the convenience of the reader (cf [14, Remark 3.2] and [21, Theorem 3.13]).

\footnotetext{
${ }^{7}$ Note This definition of $\operatorname{sing}(u)$ is different from the one used in [21]
} 
Theorem 4.6 (Theorem 2.3) Let $(W, \bar{J})$ be an almost complex 4-manifold with cylindrical ends $\left(\mathbb{R}^{ \pm} \times M^{ \pm}, \mathcal{H}^{ \pm}, J^{ \pm}\right)$and let $[\Sigma, j, \Gamma, u] \in \mathcal{M}\left(W, \bar{J}, \mathcal{H}^{+}, \mathcal{H}^{-}\right)$be a connected, simple pseudoholomorphic curve. Then

$$
[u] *[u]-\frac{1}{2} \mu(u)+\frac{1}{2} \# \Gamma_{\text {odd }}+\chi(\Sigma)-\bar{\sigma}(u)=2 \operatorname{sing}(u)
$$

where $\# \Gamma_{\text {odd }}$ denotes the number of punctures of $u$ limiting to orbits with odd ConleyZehnder indices. In particular,

$$
[u] *[u]-\frac{1}{2} \mu(u)+\frac{1}{2} \# \Gamma_{\text {odd }}+\chi(\Sigma)-\bar{\sigma}(u) \geq 0
$$

and equality occurs if and only if $u$ is an embedding and has total asymptotic selfintersection index equal to zero.

Proof We first observe that it suffices to assume that $u$ is an immersion having only transverse double points. If not we can apply results of Micallef and White [18] to find an immersion $u_{1}: \Sigma \backslash \Gamma \rightarrow W$ which is homotopic to $u$ via a homotopy that is constant outside a compact subset of $\Sigma \backslash \Gamma$, which has precisely $\delta(u)$ transverse double points and no other singularities and which is $\widetilde{J}_{1}$-holomorphic for some $\widetilde{J}_{1}$ which is homotopic to $\widetilde{J}$ via a homotopy that is constant outside a compact set. Such a homotopy leaves all the terms in (4-11) constant and it thus suffices to prove the result for such a $u_{1}$.

Proceeding with these assumptions, we compute the relative intersection number of $u$ with itself for a given trivialization $\Phi$ by pushing $u$ off of itself with a section of the normal bundle to $u$ which is asymptotic at each puncture to $\Phi(\varepsilon)$. Using standard homotopy invariance and transversality arguments, it can be shown that the zeroes of the section contribute $c_{1}^{\Phi}\left(N_{u}\right)$ to $i^{\Phi}(u, u)$ and the double points of $u$ contribute $2 \delta(u)$ to $i^{\Phi}(u, u)$. Moreover, the local asymptotic analysis in Section 3.2 shows that multiply covered ends and distinct ends approaching coverings of the same orbits contributes a total of

$$
\sum_{z \in \Gamma} i_{\infty}^{\Phi}(u ; z)+\sum_{\substack{(z, w) \in \Gamma \times \Gamma \\ z \neq w}} i_{\infty}^{\Phi}([u ; z],[u ; w])
$$

so consequently we have that

$$
i^{\Phi}(u, u)=c_{1}^{\Phi}\left(N_{u}\right)+2 \delta(u)+\sum_{z \in \Gamma} i_{\infty}^{\Phi}(u ; z)+\sum_{\substack{(z, w) \in \Gamma \times \Gamma \\ z \neq w}} i_{\infty}^{\Phi}([u ; z],[u ; w]) .
$$

(This is equivalent to [15, formula (4.1)] and to the first formula on page 110 of [21]). 
Assuming that at a puncture $z \in \Gamma^{ \pm}, u$ is asymptotic to $\gamma_{z}^{m_{z}}$, we add

$$
\begin{aligned}
& \sum_{\substack{z, w) \in \Gamma \times \Gamma \\
\gamma_{z}=\gamma_{w} \\
m_{z} m_{w}>0}} m_{z} m_{w} \max \left\{\frac{\alpha^{\Phi}\left(\gamma_{z}^{m_{z}}\right)}{\left|m_{z}\right|}, \frac{\alpha^{\Phi}\left(\gamma_{w}^{m_{w}}\right)}{\left|m_{w}\right|}\right\} \\
& =\sum_{z \in \Gamma}\left|m_{z}\right| \alpha^{\Phi}\left(\gamma_{z}^{m_{z}}\right)+\sum_{\substack{(z, w) \in \Gamma \times \Gamma \\
z \neq w \\
z \\
\gamma_{z}=\gamma_{w} \\
m_{z} m_{w}>0}} m_{z} m_{w} \max \left\{\frac{\alpha^{\Phi}\left(\gamma_{z}^{m_{z}}\right)}{\left|m_{z}\right|}, \frac{\alpha^{\Phi}\left(\gamma_{w}^{m_{w}}\right)}{\left|m_{w}\right|}\right\}
\end{aligned}
$$

to (4-12) and use the definitions of $[\cdot] *[\cdot], \delta_{\infty}(\cdot)$ and $\delta_{\infty}(\cdot, \cdot)$ to get

$$
\begin{aligned}
{[u] *[u] } & =c_{1}^{\Phi}\left(N_{u}\right)+2 \delta(u)+\sum_{z \in \Gamma}\left[2 \delta_{\infty}(u ; z)+\alpha(u ; z)+\bar{\sigma}(u ; z)-1\right] \\
& +\sum_{\substack{(z, w) \in \Gamma \times \Gamma \\
z \neq w}} \delta_{\infty}([u ; z],[u ; w]) \\
= & c_{1}^{\Phi}\left(N_{u}\right)+2 \operatorname{sing}(u)+\bar{\sigma}(u)-\# \Gamma+\sum_{z \in \Gamma} \alpha(u ; z) \\
= & c_{1}^{\Phi}\left(N_{u}\right)+2 \operatorname{sing}(u)+\bar{\sigma}(u)-\# \Gamma-\frac{1}{2} \# \Gamma_{\text {odd }}+\sum_{z \in \Gamma} \frac{1}{2} \mu^{\Phi}(u ; z) .
\end{aligned}
$$

Finally, using the properties of the relative Chern number from Lemma 4.5, we have that

$$
\begin{aligned}
c_{1}^{\Phi}\left(u^{*} T W\right) & =\chi(\Sigma \backslash \Gamma)+c_{1}^{\Phi}\left(N_{u}\right) \\
& =\chi(\Sigma)-\# \Gamma+c_{1}^{\Phi}\left(N_{u}\right)
\end{aligned}
$$

which, combined with the above formula for $[u] *[u]$, gives that

$$
[u] *[u]-\frac{1}{2} \mu(u)+\chi(\Sigma)+\frac{1}{2} \# \Gamma_{\text {odd }}-\bar{\sigma}(u)=2 \operatorname{sing}(u) .
$$

as claimed.

It is useful in certain contexts to rewrite (4-11) in terms of the virtual dimension (or Fredholm index) of the moduli space of the curve being considered. We define the index of a pseudoholomorphic curve $[\Sigma, j, \Gamma, u] \in \mathcal{M}\left(W, \bar{J}, \mathcal{H}^{+}, \mathcal{H}^{-}\right)$by the formula

$$
\operatorname{ind}(u)=\mu(u)-\chi(\Sigma)+\# \Gamma \text {. }
$$


If $u$ is simple, the index is equal to the dimension of the moduli space of unparametrized curves near $u$ for a generic choice of admissible almost complex structures on $W$ (see $[11 ; 3])$. Using this formula we can restate the adjunction formula as follows.

Corollary 4.7 Let $(W, \bar{J})$ be an almost complex 4-manifold with cylindrical ends $\left(\mathbb{R}^{ \pm} \times M^{ \pm}, \mathcal{H}^{ \pm}, J^{ \pm}\right)$and let $[\Sigma, j, \Gamma, u] \in \mathcal{M}\left(W, \bar{J}, \mathcal{H}^{+}, \mathcal{H}^{-}\right)$be a connected, simple pseudoholomorphic curve. Then

$$
[u] *[u]-\frac{1}{2}\left[\operatorname{ind}(u)-\chi(\Sigma)+\# \Gamma_{\mathrm{even}}\right]+\# \Gamma-\bar{\sigma}(u)=2 \operatorname{sing}(u) .
$$

Using the homotopy invariance of the generalized intersection number, we have the following corollary which allows us to bound the number of intersections between two pseudoholomorphic curves which are homotopic. This result is useful in the study of finite energy foliations (see eg [12]).

Corollary 4.8 Let $[\Sigma, j, \Gamma, u] \in \mathcal{M}\left(W, \bar{J}, \mathcal{H}^{+}, \mathcal{H}^{-}\right)$be a connected, simple pseudoholomorphic curve and let $\left[\Sigma^{\prime}, j^{\prime}, \Gamma^{\prime}, v\right] \in \mathcal{M}\left(W, \bar{J}, \mathcal{H}^{+}, \mathcal{H}^{-}\right)$be a second curve, distinct from $u$, which is homotopic to $u$ in $C^{\infty}\left(W, \mathcal{H}^{+}, \mathcal{H}^{-}\right)$. Then

$$
\operatorname{int}(u, v) \leq \frac{1}{2}\left[\operatorname{ind}(u)-\chi(\Sigma)+\# \Gamma_{\text {even }}\right]+\bar{\sigma}(u)-\# \Gamma+2 \operatorname{sing}(u) .
$$

\section{Cylindrical cobordisms}

In this section we will consider some applications of the intersection theory we have developed in previous sections to curves in a cylindrical cobordism. Throughout the remainder of this section $M$ will denote a 3-manifold equipped with the nondegenerate stable Hamiltonian structure $\mathcal{H}=(\lambda, \omega)$ and a compatible almost complex structure $J \in \mathcal{J}(M, \mathcal{H})$. As in previous sections, we will denote the associated $\mathbb{R}$-invariant almost complex structure on $\mathbb{R} \times M$ by $\widetilde{J}$.

It will be convenient for many of the results in this section to consider a special class of smooth asymptotically cylindrical maps. Given an asymptotically cylindrical map $(\Sigma, j, \Gamma,(a, u)) \in C^{\infty}(M, \mathcal{H})$, we say that $(\Sigma, j, \Gamma,(a, u))$ has ends which wind if there is an open neighborhood $U$ of $\Gamma$ so that $\left.u\right|_{U \backslash \Gamma}$ does not intersect any of the asymptotic limits of $u$, ie in the language of Section 3.1.4 a neighborhood of each puncture determines an asymptotically cylindrical end which winds. If $(\Sigma, j, \Gamma,(a, u))$ is an asymptotically cylindrical map with ends that wind, we can define for each puncture $z \in \Gamma$ the asymptotic winding $\operatorname{wind}_{\infty}^{\Phi}(u ; z)$ as we do in Section 3.1.4 by choosing a small clockwise loop $\rho$ around $z$ and computing the winding of $u \circ \rho$ relative to a trivialization $\Phi$ of $\xi^{\mathcal{H}}$ along the asymptotic limit of $u$ at $z$. 


\subsection{Tangency to the flow and wind $\pi$}

Let $\tilde{u}=(a, u): \Sigma \backslash \Gamma \rightarrow \mathbb{R} \times M$ be a pseudoholomorphic curve and let $\pi_{\xi^{\mathcal{H}}}: T M=$ $\mathbb{R} X_{\mathcal{H}} \oplus \xi^{\mathcal{H}} \rightarrow \xi^{\mathcal{H}}$ be the projection onto $\xi^{\mathcal{H}}$ determined by the splitting (2-1). Then the projection $\pi_{\xi_{\mathcal{H}}} \circ d u$ of the derivative of $u$ is a complex linear map

$$
\pi_{\xi^{\mathcal{H}}} \circ d u(z):\left(T_{z}(\Sigma \backslash \Gamma), j\right) \rightarrow\left(\xi_{u(z)}^{\mathcal{H}}, J\right)
$$

according to the second equation in (2-6). Thus for any $z \in \Sigma \backslash \Gamma, \pi_{\xi_{\mathcal{H}}} \circ d u(z)$ has rank 0 or 2. In the case that the Hamiltonian structure on $M$ arises from a contact form, it is shown in [8] that $\pi_{\xi_{\mathcal{H}}} \circ d u$ either vanishes identically or has isolated zeroes of finite positive order. The proof given there readily generalizes to the case of an arbitrary stable Hamiltonian structure (see Appendix A). Moreover, assuming that $\pi_{\xi_{\mathcal{H}}} \circ d u$ does not vanish identically, it follows from Corollary 3.12 that $\pi_{\xi \mathcal{H}} \circ d u$ has at most a finite number of zeroes. Following [8], we will denote the algebraic count of the zeroes of $\pi_{\xi^{\mathcal{H}}} \circ d u$ by $\operatorname{wind}_{\pi}(u)$.

Since $\pi_{\xi^{\mathcal{H}}} \circ d u$ is a section of the complex line bundle, $\operatorname{Hom}_{\mathbb{C}}\left(T_{z}(\Sigma \backslash \Gamma), u^{*} \xi^{\mathcal{H}}\right)$, the algebraic count of zeroes of $\pi_{\xi^{\mathcal{H}}} \circ d u$ is determined by the behavior of $u$ near the punctures. Indeed in [8] it is shown that

$$
\begin{aligned}
\operatorname{wind}_{\pi}(u)+d_{0}(\tilde{u}) & =\frac{1}{2}\left(\operatorname{ind}(\tilde{u})-\chi(\Sigma)+\# \Gamma_{\text {even }}\right) \\
d_{0}(\tilde{u}) & =\sum_{z \in \Gamma} d_{0}(\tilde{u} ; z)
\end{aligned}
$$

and $d_{0}(\tilde{u} ; z)$ is as defined in (3-18). Substituting into (4-14) gives the following corollary to Theorem 4.6 which will be useful for some results later in this section.

Corollary 5.1 Let $[\Sigma, j, \Gamma, \tilde{u}=(a, u)] \in \mathcal{M}(M, \mathcal{H}, J)$ be a connected, simple pseudoholomorphic curve. If $\pi_{\xi \mathcal{H}} \circ d u$ does not vanish identically, then

$$
[\tilde{u}] *[\tilde{u}]-\left[\operatorname{wind}_{\pi}(\tilde{u})+d_{0}(\tilde{u})\right]+\# \Gamma-\bar{\sigma}(\tilde{u})=2 \operatorname{sing}(\tilde{u}) .
$$

The results in this section will mostly be concerned with pseudoholomorphic curves that have no component with image contained in an orbit cylinder. We collect in the following lemma some conditions that are equivalent to this. This fact is well-known and can be deduced using Corollary 3.12 and Corollary A.3. For further discussion and proof see [8].

Lemma 5.2 Let $[\Sigma, j, \Gamma, \tilde{u}=(a, u)] \in \mathcal{M}(M, \mathcal{H}, J)$ be a pseudoholomorphic curve. The following are equivalent.

(1) $\pi_{\xi_{\mathcal{H}}} \circ d u$ does not vanish identically on any component of $\Sigma \backslash \Gamma$. 
(2) No component of the map has image contained in an orbit cylinder.

(3) No component of the map is fixed by the $\mathbb{R}$-action.

(4) The map $[\Sigma, j, \Gamma, \tilde{u}=(a, u)]$ has ends which wind, ie for each $z \in \Gamma$ and trivialization $\Phi$ of $\xi^{\mathcal{H}}$ along the asymptotic limit of $\tilde{u}$ at $z$, the asymptotic winding $\operatorname{wind}_{\infty}^{\Phi}(\tilde{u} ; z)$ is defined.

\subsection{Intersections with orbit cylinders}

In this section we compute intersection numbers of an orbit cylinder with an asymptotically cylindrical map with ends that wind. Throughout this section, we will be dealing with an asymptotically cylindrical map $(\Sigma, j, \Gamma, \tilde{u})$ with ends that wind and we will use the notation $\gamma_{z}^{m_{z}}$, with $m_{z}$ a nonzero integer and $\gamma_{z} \in \mathcal{P}^{0}(M, \mathcal{H})$ a simple periodic orbit, to indicate that at the puncture $z \in \Gamma, \tilde{u}$ is asymptotic to $\tilde{\gamma}_{z}^{m_{z}}$.

We first compute the relative intersection number.

Lemma 5.3 Let $(\Sigma, j, \Gamma, \tilde{u}=(a, u)) \in C^{\infty}(M, \mathcal{H})$ be an asymptotically cylindrical map with ends that wind and let $\tilde{\gamma}^{k}$ be the orbit cylinder over $\gamma^{k}$, with $\gamma$ a simple periodic orbit and $k$ a positive integer. Then the algebraic intersection number int $(\gamma, u)$ is well-defined and

$$
i^{\Phi}\left(\tilde{u}, \tilde{\gamma}^{k}\right)=k\left(\operatorname{int}(\gamma, u)-\sum_{\substack{z \in \Gamma \\ \gamma_{z}=\gamma}} \operatorname{wind}_{\infty}^{\Phi}(u ; z)\right) .
$$

Proof Since we assume that the map $\tilde{u}$ has ends that wind, there is a neighborhood of each puncture on which $\tilde{u}$ does not intersect $\tilde{\gamma}$ and hence on which the projected map $u$ does not intersect $\gamma$. Applying standard transversality and homotopy-invariance arguments, $u$ can therefore be perturbed on a compact set to a map with a finite number of isolated, transverse intersections with $\gamma$ and the total algebraic count of these intersections is independent of the perturbation. The intersection number $\operatorname{int}(\gamma, u)$ can then be defined to be the algebraic count of these intersections.

Using the definition of the relative intersection number and using the same reasoning that leads to Theorem 4.2 we have

$$
i^{\Phi}\left(\tilde{u}, \tilde{\gamma}^{k}\right)=\operatorname{int}\left(\tilde{\gamma}^{k}, \tilde{u}\right)+\sum_{\substack{z \in \Gamma^{+} \\ \gamma_{z}=\gamma}} i_{\infty}^{\Phi}\left(\left[\tilde{\gamma}^{k} ; \infty\right],[\tilde{u} ; z]\right)+\sum_{\substack{z \in \Gamma^{-} \\ \gamma_{z}=\gamma}} i_{\infty}^{\Phi}\left(\left[\tilde{\gamma}^{k} ; 0\right],[\tilde{u} ; z]\right) .
$$


where we consider $\tilde{\gamma}^{k}$ as a map from $S^{2} \backslash\{0, \infty\}$ with $\infty$ the positive puncture and 0 the negative puncture. Since $\tilde{\gamma}$ is fixed by the $\mathbb{R}$-action we have that $^{8}$

$$
\operatorname{int}\left(\tilde{\gamma}^{k}, \tilde{u}\right)=\operatorname{int}\left(\gamma^{k}, u\right)=k \operatorname{int}(\gamma, u) .
$$

Moreover, it follows from Lemma 3.20 and the definitions of wind $_{\text {rel }}$ and wind ${ }_{\infty}$ that for a puncture $z \in \Gamma^{+}$with $\gamma_{z}=\gamma$ we have

$$
\begin{aligned}
i_{\infty}^{\Phi}\left(\left[\tilde{\gamma}^{k} ; \infty\right],[\tilde{u} ; z]\right) & =-\frac{1}{k m_{z}} \operatorname{wind}_{\mathrm{rel}}^{\Phi}\left(m_{z} \cdot\left[\tilde{\gamma}^{k} ; \infty\right], k \cdot[\tilde{u} ; z]\right) \\
& =-\frac{1}{k m_{z}} k m_{z} \operatorname{wind}_{\infty}^{\Phi}(k \cdot[\tilde{u} ; z]) \\
& =-k \operatorname{wind}_{\infty}^{\Phi}(\tilde{u} ; z) .
\end{aligned}
$$

Similarly, for $z \in \Gamma^{-}$with $\gamma_{z}=\gamma$ we have

$$
i_{\infty}^{\Phi}\left(\left[\tilde{\gamma}^{k} ; 0\right],[\tilde{u} ; z]\right)=-k \operatorname{wind}_{\infty}^{\Phi}(\tilde{u} ; z)
$$

which completes the proof.

Next we compute the generalized intersection number of an orbit cylinder with an asymptotically cylindrical map with ends that wind. A variation on this is used in [19] to study a variation on contact homology in which the curves in the differential are required to not intersect a prescribed collection of elliptic orbits.

Corollary 5.4 Let $(\Sigma, j, \Gamma, \tilde{u}=(a, u)) \in C^{\infty}(M, \mathcal{H})$ be an asymptotically cylindrical map with ends that wind and let $\tilde{\gamma}$ be a cylinder over a simply covered periodic orbit $\gamma$. Then for $k>0$ we have

$$
\begin{aligned}
{[\tilde{u}] *\left[\tilde{\gamma}^{k}\right]=k } & (\operatorname{int}(\gamma, u) \\
& +\sum_{\substack{z \in \Gamma_{+} \\
\gamma_{z}=\gamma}} m_{z}\left[\max \left\{\frac{\alpha\left(\gamma^{m_{z}}\right)}{m_{z}}, \frac{\alpha\left(\gamma^{k}\right)}{k}\right\}-\frac{\alpha\left(\gamma^{m_{z}}\right)}{m_{z}}\right]+d_{0}(\tilde{u} ; z) \\
& \left.+\sum_{\substack{z \in \Gamma_{\bar{\gamma}} \\
\gamma_{z}=\gamma}}\left|m_{z}\right|\left[\max \left\{\frac{\alpha\left(\gamma^{m_{z}}\right)}{\left|m_{z}\right|}, \frac{\alpha\left(\gamma^{-k}\right)}{k}\right\}-\frac{\alpha\left(\gamma^{m_{z}}\right)}{\left|m_{z}\right|}\right]+d_{0}(\tilde{u} ; z)\right) .
\end{aligned}
$$

Proof The result follows from adding

$$
\sum_{\substack{z \in \Gamma_{+} \\ \gamma_{z}=\gamma}} k m_{z} \max \left\{\frac{\alpha\left(\gamma^{m_{z}}\right)}{m_{z}}, \frac{\alpha\left(\gamma^{k}\right)}{k}\right\}+\sum_{\substack{z \in \Gamma_{\bar{\gamma}} \\ \gamma_{z}=\bar{\gamma}}} k\left|m_{z}\right| \max \left\{\frac{\alpha\left(\gamma^{m_{z}}\right)}{\left|m_{z}\right|}, \frac{\alpha\left(\gamma^{-k}\right)}{k}\right\}
$$

${ }^{8}$ Note that the orientation of $M$ matters in the right-most expression since $M$ is 3-dimensional. We always orient $M$ so that $\lambda \wedge \omega$ is a positive volume form and $\mathbb{R} \times M$ is oriented so that $d x \wedge \lambda \wedge \omega>0$ where $x$ is the coordinate along $\mathbb{R}$. 
to (5-3) and using the definition of $d_{0}(\tilde{u} ; z)$ and $[\tilde{u}] *\left[\tilde{\gamma}^{k}\right]$.

We observe that in the event that the map $(\Sigma, j, \Gamma, \tilde{u})$ in this Corollary is pseudoholomorphic, then the formula given above expresses the generalized intersection number $[\tilde{u}] *\left[\tilde{\gamma}^{k}\right]$ as a sum of nonnegative terms. Indeed, the terms of the form $\max \left\{\frac{\alpha\left(\gamma^{m}\right)}{|m|}, \frac{\alpha\left(\gamma^{k}\right)}{|k|}\right\}-\frac{\alpha\left(\gamma^{m}\right)}{|m|}$ are nonnegative for any map. In the case that $\tilde{u}$ is pseudoholomorphic, the terms $d_{0}(\tilde{u} ; z)$ are nonnegative by Lemma 3.13. Moreover, when $\tilde{u}$ is pseudoholomorphic the fact that $\operatorname{int}(\gamma, u)$ is nonnegative follows from the fact from (5-4) that $\operatorname{int}(\gamma, u)=\operatorname{int}(\tilde{\gamma}, \tilde{u})$ and positivity of intersections for pseudoholomorphic curves.

In the case that $\gamma$ is a hyperbolic orbit, the iteration formulas from Lemma 3.3 simplify the formula from Corollary 5.4 somewhat. We state this result here and omit the straightforward proof.

Corollary 5.5 Let $\tilde{u}=(a, u)$ and $\gamma$ satisfy the assumptions of Corollary 5.4 and let $k>0$. If $\gamma$ is an even orbit, then

$$
[\tilde{u}] *\left[\tilde{\gamma}^{k}\right]=k\left(\operatorname{int}(\gamma, u)+\sum_{\substack{z \in \Gamma \\ \gamma_{z}=\gamma}} d_{0}(\tilde{u} ; z)\right) .
$$

If $\gamma$ is an odd, hyperbolic orbit and $k$ is even, then

$$
[\tilde{u}] *\left[\tilde{\gamma}^{k}\right]=k\left(\operatorname{int}(\gamma, u)+\sum_{\substack{z \in \Gamma \\ \gamma_{z}=\gamma}}\left[\frac{p\left(m_{z}\right)}{2}+d_{0}(\tilde{u} ; z)\right]\right)
$$

where $p(m)$ denotes the parity of $m$. If $\gamma$ is an odd, hyperbolic orbit and $k$ is odd, then

$$
[\tilde{u}] *\left[\tilde{\gamma}^{k}\right]=k \operatorname{int}(\gamma, u)+\sum_{\substack{z \in \Gamma \\ \gamma_{z}=\gamma}}\left[\frac{p\left(m_{z}\right)}{2}\left(k-\min \left\{k,\left|m_{z}\right|\right\}\right)+k d_{0}(\tilde{u} ; z)\right] .
$$

Finally, we compute the generalized intersection number of an orbit cylinder with itself.

Proposition 5.6 Let $\gamma$ be a simple periodic orbit and let $k>0$. Then

$$
\left[\tilde{\gamma}^{k}\right] *\left[\tilde{\gamma}^{k}\right]=-k p\left(\gamma^{k}\right)
$$

where $p\left(\gamma^{k}\right)$ is the parity of periodic orbit $\gamma^{k}$. 
Proof It follows immediately from the definition of the relative intersection number that $i^{\Phi}\left(\tilde{\gamma}^{k}, \tilde{\gamma}^{k}\right)$ vanishes for any trivialization $\Phi$ of $\gamma^{*} \xi^{\mathcal{H}}$. Consequently, the definition (4-3) of the generalized intersection number gives us that

$$
\begin{aligned}
{\left[\tilde{\gamma}^{k}\right] *\left[\tilde{\gamma}^{k}\right] } & =\Omega^{\Phi}\left(\tilde{\gamma}^{k}, \tilde{\gamma}^{k}\right) \\
& =k\left(\alpha^{\Phi}\left(\gamma^{k}\right)+\alpha^{\Phi}\left(\gamma^{-k}\right)\right) .
\end{aligned}
$$

If $\gamma$ is an even orbit, we have from (3-5) that

$$
\alpha^{\Phi}\left(\gamma^{k}\right)+\alpha^{\Phi}\left(\gamma^{-k}\right)=k \alpha^{\Phi}(\gamma)-k \alpha^{\Phi}(\gamma)=0
$$

so $\left[\tilde{\gamma}^{k}\right] *\left[\tilde{\gamma}^{k}\right]=0=-k p\left(\gamma^{k}\right)$ in this case. If $\gamma$ is an odd hyperbolic orbit, we have from (3-6) that

$$
\begin{aligned}
\alpha^{\Phi}\left(\gamma^{k}\right)+\alpha^{\Phi}\left(\gamma^{-k}\right) & =k \alpha^{\Phi}(\gamma)+\frac{k-p(k)}{2}-k \alpha^{\Phi}(\gamma)+\frac{-k-p(-k)}{2} \\
& =-p(k)=-p\left(\gamma^{k}\right),
\end{aligned}
$$

so $\left[\tilde{\gamma}^{k}\right] *\left[\tilde{\gamma}^{k}\right]=-k p\left(\gamma^{k}\right)$ in this case as well. Finally, if $\gamma$ is elliptic, it follows from (3-7) that there exists an irrational number $\theta$ so that

$$
\begin{aligned}
\alpha^{\Phi}\left(\gamma^{k}\right)+\alpha^{\Phi}\left(\gamma^{-k}\right) & =\lfloor k \theta\rfloor+\lfloor-k \theta\rfloor \\
& =\lfloor k \theta\rfloor-\lceil k \theta\rceil=-1
\end{aligned}
$$

so we have $\left[\tilde{\gamma}^{k}\right] *\left[\tilde{\gamma}^{k}\right]=-k=-k p\left(\gamma^{k}\right)$ for elliptic orbits as well.

This computation has an application to curves in $\mathbb{R} \times M$ equipped with a non- $\mathbb{R}-$ invariant almost complex structure. We consider a stable Hamiltonian structure $\mathcal{H}=$ $(\lambda, \omega)$ on $M$ and let $c \cdot \mathcal{H}$ denote the stable Hamiltonian structure $c \cdot \mathcal{H}=(c \cdot \lambda, c \cdot \omega)$. Consider $\mathbb{R} \times M$ equipped with an almost complex structure $\bar{J}$ which agrees on $[1,+\infty) \times M$ with a standard cylindrical $\widetilde{J}_{1}$ for $c_{1} \cdot \mathcal{H}$ and agrees on $(-\infty,-1] \times M$ with a standard cylindrical $\widetilde{J}_{2}$ for $c_{2} \cdot \mathcal{H}$. If $\gamma^{k}$ is a periodic orbit for the structure $\mathcal{H}$, it is also a periodic orbit for the structure $c_{i} \cdot \mathcal{H}$ and the Conley-Zehnder index of $\gamma^{k}$ is the same relative to all three structures. We can consider the orbit cylinder $\tilde{\gamma}^{k}$, which in general will not be $\bar{J}$-holomorphic, but is still a smooth asymptotically cylindrical map. The preceding proposition still applies and implies that $\tilde{\gamma}^{k}$ has a negative (generalized) self-intersection number if $\gamma^{k}$ is an odd orbit. By the positivity of intersections for the generalized intersection number (Theorem 4.4) and the homotopy invariance of the generalized intersection number, we can conclude that there is at most one $\bar{J}$-holomorphic curve in the homotopy class of the orbit cylinder $\tilde{\gamma}^{k}$. 


\subsection{Counting intersections}

In this section we will compute generalized intersection number of two maps in terms of data that is entirely determined by the $M$-components of the maps: namely the intersection numbers of each map with the asymptotic limits of the other, the asymptotic winding invariants and the asymptotic data. We then identify necessary and sufficient conditions for the generalized intersection number of two pseudoholomorphic curves to be zero. Finally, we examine a set of necessary and sufficient conditions for the projections of two pseudoholomorphic curves into the three manifold to not intersect.

We recall that there is an $\mathbb{R}$-action on maps in cylindrical cobordisms, defined by shifting in the $\mathbb{R}$-direction of $\mathbb{R} \times M$. We will denote this action by $c \cdot \tilde{u}=(a+c, u)$ or by $\tilde{u}_{c}=(a+c, u)$ where $\tilde{u}=(a, u): \Sigma \backslash \Gamma \rightarrow \mathbb{R} \times M$ is an asymptotically cylindrical map.

We also recall that [2] defines what it means for a pseudoholomorphic curve to converge to a pseudoholomorphic building (see CHC1-CHC2 in [2, Section 7.3]). The definition of convergence of pseudoholomorphic curves given there can be generalized in straightforward way to asymptotically cylindrical maps converging to asymptotically cylindrical buildings.

Lemma 5.7 Let $\left(\Sigma_{k}, j_{k}, \Gamma_{k}, \tilde{u}_{k}\right),\left(\Sigma_{k}^{\prime}, j_{k}^{\prime}, \Gamma_{k}^{\prime}, \tilde{v}_{k}\right) \in C^{\infty}(M, \mathcal{H})$ be sequences of asymptotically cylindrical maps so that the disjoint union $\tilde{u}_{k}+\tilde{v}_{k}$ converges in the sense of [2] to a 2-level building $\left(\tilde{u}_{\infty, 1} \odot \tilde{u}_{\infty, 2}\right)+\left(\tilde{v}_{\infty, 1} \odot \tilde{v}_{\infty, 2}\right)$. Then

$$
\lim _{k \rightarrow \infty} i^{\Phi}\left(\tilde{u}_{k}, \tilde{v}_{k}\right)=i^{\Phi}\left(\tilde{u}_{\infty, 1}, \tilde{v}_{\infty, 1}\right)+i^{\Phi}\left(\tilde{u}_{\infty, 2}, \tilde{v}_{\infty, 2}\right) .
$$

We remark that the result and proof here easily adapt to the case where the limit building has more than 2 levels and when the limiting curves are mapped into a splitting symplectic manifold.

Proof According to definition of convergence in [2], our assumptions imply that there exist constants $c_{k}$ and $d_{k}$ and holomorphic reparametrizations $\phi_{k, i}: \Sigma_{k} \amalg \Sigma_{k}^{\prime} \rightarrow$ $\Sigma_{k} \amalg \Sigma_{k}^{\prime}$ so that $c_{k} \cdot\left(\tilde{u}_{k}+\tilde{v}_{k}\right) \circ \phi_{k, 1}$ converges in $C_{\text {loc }}^{\infty}$ to $\tilde{u}_{\infty, 1}+\tilde{v}_{\infty, 1}$ and so that $d_{k} \cdot\left(\tilde{u}_{k}+\tilde{v}_{k}\right) \circ \phi_{k, 2}$ converges in $C_{\text {loc }}^{\infty}$ to $\tilde{u}_{\infty, 2}+\tilde{v}_{\infty, 2}$. Moreover, we can compactify $(\mathbb{R} \times M) \odot(\mathbb{R} \times M)$ and identify it with $[0,1] \times M$ (equipped with a $C^{0}$ structure) and can choose a sequence of identifications of the compactification of $\mathbb{R} \times M$ with $[0,1] \times M$ and diffeomorphisms $\psi_{k}: \Sigma_{k} \amalg \Sigma_{k}^{\prime} \rightarrow \Sigma_{k} \amalg \Sigma_{k}^{\prime}$ in such a way that $\left(\tilde{u}_{k}+\tilde{v}_{k}\right) \circ \psi_{k}$ converges uniformly to $\left(\tilde{u}_{\infty, 1} \odot \tilde{u}_{\infty, 2}\right)+\left(\tilde{v}_{\infty, 1} \odot \tilde{v}_{\infty, 2}\right)$, all viewed as maps into $[0,1] \times M$. It then follows from the properties of intersection numbers and the definition of the relative intersection number that for large $k$ we have $i^{\Phi}\left(\tilde{u}_{k}, \tilde{v}_{k}\right)=i^{\Phi}\left(\tilde{u}_{\infty, 1}, \tilde{v}_{\infty, 1}\right)+i^{\Phi}\left(\tilde{u}_{\infty, 2}, \tilde{v}_{\infty, 2}\right)$ as claimed. 
As an immediate application of this, we have the following computation of the generalized intersection number of two asymptotically cylindrical maps with ends that wind.

Theorem 5.8 Let $(\Sigma, j, \Gamma, \tilde{u}=(a, u)),\left(\Sigma^{\prime}, j^{\prime}, \Gamma^{\prime}, \tilde{v}=(b, v)\right) \in C^{\infty}(M, \mathcal{H})$ be asymptotically cylindrical maps with ends that wind. Assume that at $z \in \Gamma$ that $\tilde{u}$ is asymptotic to $\gamma_{z}^{m_{z}}$ and that at $w \in \Gamma^{\prime} \tilde{v}$ is asymptotic to $\gamma_{w}^{m_{w}}$ with $m_{z}, m_{w}$ nonzero integers and $\gamma_{z}, \gamma_{w} \in \mathcal{P}^{0}(M, \mathcal{H})$, simple periodic orbits. Then

$$
\begin{aligned}
{[\tilde{u}] *[\tilde{v}] } & =\sum_{w \in \Gamma_{+}^{\prime}}\left|m_{w}\right|\left(\operatorname{int}\left(\gamma_{w}, u\right)+\sum_{\substack{z \in \Gamma_{+}^{+} \\
\gamma_{z}=\gamma_{w}}}\left|m_{z}\right| \bar{\Lambda}\left(\frac{\alpha\left(\gamma_{w}^{m_{w}}\right)}{\left|m_{w}\right|}, \frac{\alpha\left(\gamma_{z}^{m_{z}}\right)}{\left|m_{z}\right|}\right)+d_{0}(\tilde{u} ; z)\right) \\
(5-5) \quad & +\sum_{z \in \Gamma_{-}}\left|m_{z}\right|\left(\begin{array}{c}
\operatorname{int}\left(\gamma_{z}, v\right)+\sum_{\substack{w \in \Gamma^{\prime} \\
\gamma_{w}=\gamma_{z}}}\left|m_{w}\right| \bar{\Lambda}\left(\frac{\alpha\left(\gamma_{z}^{m_{z}}\right)}{\left|m_{z}\right|}, \frac{\alpha\left(\gamma_{w}^{m_{w}}\right)}{\left|m_{w}\right|}\right)+d_{0}(\tilde{v} ; w)
\end{array}\right) \\
& +\sum_{\substack{(z, w) \in \Gamma_{-} \times \Gamma_{+}^{\prime} \\
\gamma_{z}=\gamma_{w}}}\left|m_{w}\right| d_{0}(\tilde{u} ; z)+\left|m_{z}\right| d_{0}(\tilde{v} ; w)-\left|m_{w} m_{z}\right|\left[\frac{\alpha\left(\gamma_{z}^{m_{z}}\right)}{\left|m_{z}\right|}+\frac{\alpha\left(\gamma_{w}^{m_{w}}\right)}{\left|m_{w}\right|}\right]
\end{aligned}
$$

where

$$
\bar{\Lambda}(x, y):=\max \{x, y\}-y \text {. }
$$

Proof We apply the $\mathbb{R}$-action to $\tilde{u}$ and note that

$$
\lim _{c \rightarrow \infty}(c \cdot \tilde{u})+\tilde{v}=\left(\tilde{u} \odot\left[+_{z \in \Gamma_{-}} \tilde{\gamma}_{z}^{-m_{z}}\right]\right)+\left(\left[+_{w \in \Gamma_{+}^{\prime}} \tilde{\gamma}_{w}^{m_{w}}\right] \odot \tilde{v}\right)
$$

where the limit is to be understood in the sense of [2] and "+" denotes the disjoint union of the maps. Consequently, the homotopy invariance of the relative intersection number along with Lemma 5.7 imply that

$$
i^{\Phi}(\tilde{u}, \tilde{v})=\lim _{c \rightarrow \infty} i^{\Phi}(c \cdot \tilde{u}, \tilde{v})=\sum_{w \in \Gamma_{+}^{\prime}} i^{\Phi}\left(\tilde{u}, \tilde{\gamma}_{w}^{m_{w}}\right)+\sum_{z \in \Gamma_{-}} i^{\Phi}\left(\tilde{v}, \tilde{\gamma}_{z}^{-m_{z}}\right) .
$$

Applying Lemma 5.3 and the definition of $d_{0}(\tilde{u} ; z)$, we have that

$$
i^{\Phi}\left(\tilde{u}, \tilde{\gamma}_{w}^{m_{w}}\right)=m_{w}\left(\operatorname{int}\left(\gamma_{w}, u\right)-\sum_{\substack{z \in \Gamma \\ \gamma_{z}=\gamma_{w}}} \operatorname{wind}_{\infty}^{\Phi}(u ; z)\right)
$$




$$
=m_{w}\left(\operatorname{int}\left(\gamma_{w}, u\right)+\sum_{\substack{z \in \Gamma \\ \gamma_{z}=\gamma_{w}}}\left[d_{0}(\tilde{u} ; z)-\alpha^{\Phi}\left(\gamma_{z}^{m_{z}}\right)\right]\right)
$$

and similarly

$$
i^{\Phi}\left(\tilde{v}, \tilde{\gamma}_{z}^{-m_{z}}\right)=\left|m_{z}\right|\left(\operatorname{int}\left(\gamma_{z}, v\right)+\sum_{\substack{w \in \Gamma^{\prime} \\ \gamma_{w}=\gamma_{z}}}\left[d_{0}(\tilde{v} ; w)-\alpha^{\Phi}\left(\gamma_{w}^{m_{w}}\right)\right]\right)
$$

Substituting these formulas in (5-7) and adding

$$
\Omega^{\Phi}(\tilde{u}, \tilde{v})=\sum_{\substack{(z, w) \in \Gamma \times \Gamma^{\prime} \\ \gamma_{z}=\gamma_{w} \\ m_{z} m_{w}>0}} m_{z} m_{w} \max \left\{\frac{\alpha\left(\gamma_{z}^{m_{z}}\right)}{\left|m_{z}\right|}, \frac{\alpha\left(\gamma_{w}^{m_{w}}\right)}{\left|m_{w}\right|}\right\}
$$

to both sides gives

$$
\begin{aligned}
{[\tilde{u}] *[\tilde{v}]=} & i^{\Phi}(\tilde{u}, \tilde{v})+\Omega^{\Phi}(\tilde{u}, \tilde{v}) \\
= & \sum_{w \in \Gamma_{+}^{\prime}}\left|m_{w}\right|\left(\operatorname{int}\left(\gamma_{w}, u\right)+\sum_{\substack{z \in \Gamma_{+} \\
\gamma_{z}=\gamma_{w}}}\left|m_{z}\right| \bar{\Lambda}\left(\frac{\alpha\left(\gamma_{w}^{m w}\right)}{\left|m_{w}\right|}, \frac{\alpha\left(\gamma_{z}^{m_{z}}\right)}{\left|m_{z}\right|}\right)+d_{0}(\tilde{u} ; z)\right) \\
& +\sum_{z \in \Gamma_{-}}\left|m_{z}\right|\left(\operatorname{int}\left(\gamma_{z}, v\right)+\sum_{\substack{w \in \Gamma_{\bar{w}}^{\prime} \\
\gamma_{z}}}\left|m_{w}\right| \bar{\Lambda}\left(\frac{\alpha\left(\gamma_{z}^{m_{z}}\right)}{\left|m_{z}\right|}, \frac{\alpha\left(\gamma_{w}^{m_{w}}\right)}{\left|m_{w}\right|}\right)+d_{0}(\tilde{v} ; w)\right) \\
& +\sum_{(z, w) \in \Gamma_{-} \times \Gamma_{+}^{\prime}}\left|m_{w}\right| d_{0}(\tilde{u} ; z)+\left|m_{z}\right| d_{0}(\tilde{v} ; w)-\left|m_{w} m_{z}\right|\left[\frac{\alpha\left(\gamma_{z}^{m_{z}}\right)}{\left|m_{z}\right|}+\frac{\alpha\left(\gamma_{w}^{m_{w}}\right)}{\left|m_{w}\right|}\right]
\end{aligned}
$$

as claimed, where we have abbreviated $\bar{\Lambda}(x, y)=\max \{x, y\}-y$.

We note that in the case that the maps $\tilde{u}$ and $\tilde{v}$ from this theorem are pseudoholomorphic that (5-5) expresses the generalized intersection number $[\tilde{u}] *[\tilde{v}]$ as a sum of nonnegative terms. Indeed the nonnegativity of each of the terms is discussed following Corollary 5.4, with the exception of the terms of the form

$$
-\left|m_{w} m_{z}\right|\left[\frac{\alpha\left(\gamma_{z}^{m_{z}}\right)}{\left|m_{z}\right|}+\frac{\alpha\left(\gamma_{w}^{m_{w}}\right)}{\left|m_{w}\right|}\right] .
$$


The fact that these terms contribute nonnegatively to $[\tilde{u}] *[\tilde{v}]$ is addressed in the proof of Corollary 5.9 below.

The fact that (5-5) expresses $[\tilde{u}] *[\tilde{v}]$ for pseudoholomorphic curves as a sum of nonnegative terms allows us to prove the following result which gives necessary and sufficient conditions for two curves to have generalized intersection number equal to zero.

Corollary 5.9 Let $[\Sigma, j, \Gamma, \tilde{u}=(a, u)]$ and $\left[\Sigma^{\prime}, j^{\prime}, \Gamma^{\prime}, \tilde{v}=(b, v)\right] \in \mathcal{M}(M, \mathcal{H}, J)$ be pseudoholomorphic curves and assume that no component of $\tilde{u}$ or $\tilde{v}$ lies in an orbit cylinder. Then the following are equivalent:

(1) The generalized intersection number $[\tilde{u}] *[\tilde{v}]=0$.

(2) All of the following hold:

(a) The map $u$ does not intersect any of the positive asymptotic limits of $v$.

(b) The map $v$ does not intersect any of the negative asymptotic limits of $u$.

(c) If $\gamma$ is a periodic orbit so that at $z \in \Gamma, \tilde{u}$ is asymptotic to $\gamma^{m_{z}}$ and at $w \in \Gamma^{\prime}, \tilde{v}$ is asymptotic to $\gamma^{m_{w}}$, then:

(i) If $m_{z}$ and $m_{w}$ are both positive then $d_{0}(\tilde{u} ; z)=0$ and

$$
\frac{\alpha\left(\gamma^{m_{z}}\right)}{m_{z}} \geq \frac{\alpha\left(\gamma^{m_{w}}\right)}{m_{w}} .
$$

(ii) If $m_{z}$ and $m_{w}$ are both negative then $d_{0}(\tilde{v} ; w)=0$ and

$$
\frac{\alpha\left(\gamma^{m w}\right)}{\left|m_{w}\right|} \geq \frac{\alpha\left(\gamma^{m_{z}}\right)}{\left|m_{z}\right|} .
$$

(iii) If $m_{z}<0$ and $m_{w}>0$ then $d_{0}(\tilde{u} ; z)=d_{0}(\tilde{v} ; w)=0$ and $\gamma^{m_{z}}$ and $\gamma^{m_{w}}$ are both even orbits; or equivalently

$$
\frac{\operatorname{wind}_{\infty}(\tilde{u} ; z)}{m_{z}}=\frac{\operatorname{wind}_{\infty}(\tilde{v} ; w)}{m_{w}} .
$$

(3) All of the following hold:

(a) The map $u$ does not intersect any of the asymptotic limits of $v$.

(b) The map $v$ does not intersect any of the asymptotic limits of $u$.

(c) If $\gamma$ is a periodic orbit so that at $z \in \Gamma, \tilde{u}$ is asymptotic to $\gamma^{m_{z}}$ and at $w \in \Gamma^{\prime}, \tilde{v}$ is asymptotic to $\gamma^{m_{w}}$, then $d_{0}(\tilde{u} ; z)=0, d_{0}(\tilde{v} ; w)=0$. Further

(i) if $\gamma$ is elliptic, then $m_{z}$ and $m_{w}$ have the same sign and

$$
\frac{\alpha\left(\gamma^{m_{z}}\right)}{m_{z}}=\frac{\alpha\left(\gamma^{m_{w}}\right)}{m_{w}} \text {. }
$$

(ii) if $\gamma$ is odd, hyperbolic then either $m_{z}$ and $m_{w}$ are both even or $m_{z}=$ $m_{w}$. 
Proof As discussed preceding the statement of this corollary, this will follow from Theorem 5.8 and in particular the fact that Theorem 5.8 gives an expression of $[\tilde{u}] *[\tilde{v}]$ as a sum of nonnegative quantities. Indeed, as discussed following Corollary 5.4, it follows from (5-4) that the terms involving intersections of the projected maps $u$ and $v$ with periodic orbits are nonnegative. Moreover, Lemma 3.13 establishes that the $d_{0}$ terms are nonnegative, while it is clear that each other grouped term on the right hand side of equation (5-5) is nonnegative except possibly for the term in the final sum of the form

$$
\Delta_{1}(\gamma, m, n):=-|m n|\left[\frac{\alpha\left(\gamma^{m}\right)}{|m|}+\frac{\alpha\left(\gamma^{n}\right)}{|n|}\right]
$$

where here $m$ and $n$ have opposite sign. The nonnegativity of this term will follow from Lemma 3.3. Without loss of generality, assume that $m>0$ and $n<0$. So that the expression takes the form

$$
\Delta_{1}(\gamma, m, n)=m n\left[\frac{\alpha\left(\gamma^{m}\right)}{m}+\frac{\alpha\left(\gamma^{n}\right)}{-n}\right]
$$

If $\gamma$ is an even orbit then (3-5) gives us

$$
\Delta_{1}(\gamma, m, n)=m n[\alpha(\gamma)-\alpha(\gamma)]=0 .
$$

If $\gamma$ is odd, hyperbolic, (3-6) gives us

$$
\Delta_{1}(\gamma, m, n)=\frac{1}{2}[m p(n)-n p(m)]
$$

which is nonnegative since $m>0$ and $n<0$ and equals zero if and only if both $m$ and $n$ are even. Finally if $\gamma$ is elliptic, by (3-7) there is an irrational $\theta$ so that

$$
\begin{aligned}
\Delta_{1}(\gamma, m, n) & =n\lfloor m \theta\rfloor-m\lfloor n \theta\rfloor \\
& \geq\lceil n m \theta\rceil-\lfloor n m \theta\rfloor=1,
\end{aligned}
$$

where the inequality follows from the assumptions that $n<0, m>0$ and that $\theta$ is irrational. To summarize, we have seen that for $m$ and $n$ integers with opposite sign, we have that

$$
-|m n|\left[\frac{\alpha\left(\gamma^{m}\right)}{|m|}+\frac{\alpha\left(\gamma^{n}\right)}{|n|}\right] \geq 0
$$

and equality occurs if and only if $\gamma^{m}$ and $\gamma^{n}$ are both even orbits.

Given this, it is clear from (5-5) that $[\tilde{u}] *[\tilde{v}]=0$ if and only if:

- $\operatorname{int}\left(\gamma_{w}, u\right)=0$ for all $w \in \Gamma_{+}^{\prime}$,

- $\operatorname{int}\left(\gamma_{z}, v\right)=0$ for all $z \in \Gamma_{-}$,

- $d_{0}(\tilde{u} ; z)=0$ and

$$
\max \left\{\frac{\alpha\left(\gamma_{z}^{m_{z}}\right)}{\left|m_{z}\right|}, \frac{\alpha\left(\gamma_{w}^{m_{w}}\right)}{\left|m_{w}\right|}\right\}-\frac{\alpha\left(\gamma_{z}^{m_{z}}\right)}{m_{z}}=0
$$


for all $(z, w) \in \Gamma_{+} \times \Gamma_{+}^{\prime}$ with $\gamma_{z}=\gamma_{w}$,

- $d_{0}(\tilde{v} ; w)=0$ and

$$
\max \left\{\frac{\alpha\left(\gamma_{z}^{m_{z}}\right)}{\left|m_{z}\right|}, \frac{\alpha\left(\gamma_{w}^{m_{w}}\right)}{\left|m_{w}\right|}\right\}-\frac{\alpha\left(\gamma_{w}^{m_{w}}\right)}{m_{w}}=0
$$

for all $(z, w) \in \Gamma_{-} \times \Gamma_{-}^{\prime}$ with $\gamma_{z}=\gamma_{w}$ and

- $d_{0}(\tilde{u} ; z)=d_{0}(\tilde{v} ; w)=0$ and

$$
-\left|m_{z} m_{w}\right|\left[\frac{\alpha\left(\gamma_{z}^{m_{z}}\right)}{\left|m_{z}\right|}+\frac{\alpha\left(\gamma_{w}^{m_{w}}\right)}{\left|m_{w}\right|}\right]=0
$$

for all $(z, w) \in \Gamma_{-} \times \Gamma_{+}^{\prime}$ with $\gamma_{z}=\gamma_{w}$.

The discussion of the previous paragraph tells us that (5-8) is true if and only if $\gamma_{z}^{m_{z}}$ and $\gamma_{w}^{m_{w}}$ are both even orbits. Moreover, we have from Lemma 3.13 together with $m_{z}<0, m_{w}>0$ and the previously observed nonnegativity of the left-hand-side of (5-8) that

$$
\frac{\operatorname{wind}_{\infty}(\tilde{v} ; w)}{m_{w}} \leq \frac{\alpha\left(\gamma_{w}^{m} w\right)}{m_{w}} \leq \frac{\alpha\left(\gamma_{z}^{m_{z}}\right)}{m_{z}} \leq \frac{\operatorname{wind}_{\infty}(\tilde{u} ; z)}{m_{z}}
$$

in general. This together with the definition (3-18) of $d_{0}$ tells us that the last listed condition above, that is $d_{0}(\tilde{u} ; z)=d_{0}(\tilde{v} ; w)=0$ and (5-8), is true precisely when

$$
\frac{\operatorname{wind}_{\infty}(\tilde{v} ; w)}{m_{w}}=\frac{\operatorname{wind}_{\infty}(\tilde{u} ; z)}{m_{z}}
$$

so the two conditions given in item 2(c)iii are equivalent. The fact that (1) $\Longleftrightarrow$ (2) in the statement of the corollary follows immediately then from the above list of conditions.

To see that (2) $\Longleftrightarrow$ (3), we use the symmetry of the generalized intersection number with the asymmetry of statement (2). Indeed if (2) is true as written, then (1) is true and thus (2) will be true with the roles of $\tilde{u}$ and $\tilde{v}$ reversed. We immediately find that (2) holds precisely when

- $\operatorname{int}\left(\gamma_{w}, u\right)=0$ for all $w \in \Gamma^{\prime}$,

- $\operatorname{int}\left(\gamma_{z}, v\right)=0$ for all $z \in \Gamma$,

- $d_{0}(\tilde{u} ; z)=d_{0}(\tilde{v} ; w)=0$ and

$$
\frac{\alpha\left(\gamma_{z}^{m z}\right)}{\left|m_{z}\right|}=\frac{\alpha\left(\gamma_{w}^{m w}\right)}{\left|m_{w}\right|}
$$

for all $(z, w) \in \Gamma \times \Gamma^{\prime}$ with $\gamma_{z}=\gamma_{w}$ and $m_{z} m_{w}>0$, and 
- $d_{0}(\tilde{u} ; z)=d_{0}(\tilde{v} ; w)=0$ and

$$
-\left|m_{z} m_{w}\right|\left[\frac{\alpha\left(\gamma_{z}^{m_{z}}\right)}{\left|m_{z}\right|}+\frac{\alpha\left(\gamma_{w}^{m_{w}}\right)}{\left|m_{w}\right|}\right]=0
$$

for all $(z, w) \in \Gamma \times \Gamma^{\prime}$ with $\gamma_{z}=\gamma_{w}$ and $m_{z} m_{w}<0$.

Using (3-5) we see that

$$
\frac{\alpha\left(\gamma^{m}\right)}{|m|}=\frac{\alpha\left(\gamma^{n}\right)}{|n|}
$$

for any $m$ and $n$ with $m n>0$ if $\gamma$ is even and using (3-6) we see that

$$
\frac{\alpha\left(\gamma^{m}\right)}{|m|}=\frac{\alpha\left(\gamma^{n}\right)}{|n|} \Longleftrightarrow \frac{p(m)}{m}=\frac{p(n)}{n}
$$

if $\gamma$ is odd hyperbolic. This last statement is true exactly when $m$ and $n$ are both even or $m$ and $n$ are equal. These observations with the discussion of the first paragraph shows that the conditions listed above are equivalent to those listed in (3), so we see that $(2) \Longleftrightarrow(3)$.

We note that if $[\tilde{u}] *[\tilde{v}]=0$ then $[\tilde{u}] *[c \cdot \tilde{v}]=0$ for all $c \in \mathbb{R}$ and in particular, Theorem 4.4 implies that the actual algebraic intersection number $\operatorname{int}(\tilde{u}, c \cdot \tilde{v})$ is zero for all $c \in \mathbb{R}$, provided $\tilde{u}$ and $\tilde{v}$ do not have any components with images that differ by an $\mathbb{R}$-shift. By positivity of intersections, this implies that $\tilde{u}$ is disjoint from every $\mathbb{R}$-translate of $\tilde{v}$ which implies that the projected curves $u$ and $v$ in the 3-manifold $M$ either have identical image or do not intersect. Thus the preceding corollary gives sufficient conditions for the projected curves $u$ and $v$ to not intersect. The converse is not true, as is it possible that projected pseudoholomorphic maps $u$ and $v$ do not intersect, but still have $[\tilde{u}] *[\tilde{v}] \neq 0$ since the asymptotic intersection number of the two curves could be nonzero. However, since $\mathbb{R}$-shifting a curve changes the asymptotic description from Theorem 3.7 in a predictable way, it is possible to use Theorem 5.8 to identify necessary and sufficient conditions for two projected pseudoholomorphic curves to not intersect.

The key element in understanding this problem is the following lemma concerning the behavior of the total asymptotic intersection number of two curves under $\mathbb{R}$-shifting.

Lemma 5.10 Let $[\Sigma, j, \Gamma, \tilde{u}]$ and $\left[\Sigma^{\prime}, j^{\prime}, \Gamma^{\prime}, \tilde{v}\right] \in \mathcal{M}(M, \mathcal{H}, J)$ be asymptotically cylindrical pseudoholomorphic curves and let $\tilde{v}_{c}=(b+c, v)$ be the curve resulting from translating $\tilde{v}$ in the $\mathbb{R}$-coordinate by $c$. Assume no component of $\tilde{u}$ or $\tilde{v}$ has image contained in an orbit cylinder. Then the algebraic intersection number $\operatorname{int}\left(\tilde{u}, \tilde{v}_{c}\right)$ 
and the total asymptotic intersection index $\delta_{\infty}\left(\tilde{u}, \tilde{v}_{c}\right)$ are defined for all but a finite number of values of $c \in \mathbb{R}$. Moreover

$$
\delta_{\infty}\left(\tilde{u}, \tilde{v}_{c}\right) \geq \sum_{\substack{(z, w) \in \Gamma^{\prime} \times \Gamma^{\prime} \\ \gamma_{z}=\gamma_{w} \\ m_{z} m_{w}>0}} m_{z} m_{w}\left(\max \left\{\frac{\alpha(\tilde{u} ; z)}{\left|m_{z}\right|}, \frac{\alpha(\tilde{v} ; w)}{\left|m_{w}\right|}\right\}-\max \left\{\frac{\operatorname{wind}_{\infty}(\tilde{u} ; z)}{\left|m_{z}\right|}, \frac{\operatorname{wind}_{\infty}(\tilde{v} ; w)}{\left|m_{w}\right|}\right\}\right),
$$

with equality occurring for all but a finite number of the values of $c \in \mathbb{R}$ for which $\delta_{\infty}\left(\tilde{u}, \tilde{v}_{c}\right)$ is defined. Furthermore,

$$
\begin{aligned}
\operatorname{int}\left(\tilde{u}, \tilde{v}_{c}\right) \leq & {[\tilde{u}] *[\tilde{v}] } \\
& -\sum_{\substack{(z, w) \in \Gamma \times \Gamma^{\prime} \\
\gamma_{z}=\gamma_{w} \\
m_{z} m_{w}>0}} m_{z} m_{w}\left(\max \left\{\frac{\alpha(\tilde{u} ; z)}{\left|m_{z}\right|}, \frac{\alpha(\tilde{v} ; w)}{\left|m_{w}\right|}\right\}-\max \left\{\frac{\operatorname{wind}_{\infty}(\tilde{u} ; z)}{\left|m_{z}\right|}, \frac{\operatorname{wind}_{\infty}(\tilde{v} ; w)}{\left|m_{w}\right|}\right\}\right)
\end{aligned}
$$

and strict inequality occurs for at most a finite set of values of $c \in \mathbb{R}$ for which int $\left(\tilde{u}, \tilde{v}_{c}\right)$ is defined.

Proof There will be values of $c$ for which the algebraic intersection number int $\left(\tilde{u}, \tilde{v}_{c}\right)$ and the total asymptotic intersection index $\delta_{\infty}\left(\tilde{u}, \tilde{v}_{c}\right)$ will not be defined if there are components of $\tilde{u}$ and $\tilde{v}$ which project to the same image in the three-manifold, but it is clear the lifted maps $\tilde{u}$ and $\tilde{v}_{c}$ can have components with identical image in $\mathbb{R} \times M$ only for finite number of values of $c \in \mathbb{R}$, since we assume that no components of the curves are fixed by the $\mathbb{R}$-action.

We consider a pair of end models $(\Sigma, j, z, \tilde{u})$ and $\left(\Sigma^{\prime}, j^{\prime}, w, \tilde{v}=(b, v)\right)$ and assume that there is a $\gamma$ so that $\tilde{u}$ is asymptotic to $\gamma^{m}$ and $\tilde{v}$ is asymptotic to $\gamma^{n}$ with $m$ and $n$ having the same sign. Assume we have the asymptotic representations

$$
\begin{gathered}
\tilde{u}(\phi(s, t))=\left(m \tau s, \exp _{\gamma^{m}(t)} e^{\lambda_{1} s}\left[e_{1}(t)+r_{1}(s, t)\right]\right) \\
\tilde{v}\left(\psi\left(s^{\prime}, t^{\prime}\right)\right)=\left(n \tau s^{\prime}, \exp _{\gamma^{n}\left(t^{\prime}\right)} e^{\lambda_{2} s^{\prime}}\left[e_{2}\left(t^{\prime}\right)+r_{2}\left(s^{\prime}, t^{\prime}\right)\right]\right) .
\end{gathered}
$$

with the $\lambda_{i} / e_{i}$ eigenvalues/eigenvectors of the appropriate asymptotic operators and the $r_{i}$ converging exponentially to 0 as $s \rightarrow \infty$. Then, according to Lemma 3.20 and Lemma 3.15, we have that

$$
i^{\Phi}([\tilde{u} ; z],[\tilde{v} ; w]) \geq-m n \max \left\{\frac{\operatorname{wind}_{\infty}^{\Phi}(\tilde{u} ; z)}{|m|}, \frac{\operatorname{wind}_{\infty}^{\Phi}(\tilde{v} ; w)}{|n|}\right\}
$$

with strict inequality occurring only if there is an integer $j$ so that

$$
e_{1}(n t)=e_{2}\left(m t+\frac{j}{m n}\right)
$$


for all $t \in S^{1}$. Considering the shifted end

$$
\tilde{v}_{c}=(b+c, v)
$$

we have, for $\psi_{c}(s, t):=\psi\left(s+\frac{c}{n \tau}, t\right)$ that

$$
\begin{aligned}
\tilde{v}_{c}\left(\psi_{c}(s, t)\right) & =\left(n \tau s, \exp _{\gamma^{n}(t)} e^{\lambda_{2}\left(s+\frac{c}{n \tau}\right)}\left[e_{2}(t)+r_{2}\left(s+\frac{c}{n \tau}, t\right)\right]\right. \\
& =\left(n \tau s, \exp _{\gamma^{n}(t)} e^{\lambda_{2} s}\left[e^{c \frac{\lambda}{n \tau}} e_{2}(t)+r_{3}(s, t)\right]\right.
\end{aligned}
$$

with $r_{3}(s, t)=e^{c \frac{\lambda}{n \tau}} r_{2}\left(s+\frac{c}{n \tau}\right)$ converging exponentially to zero as $s \rightarrow \infty$. Thus, $\mathbb{R}$-shifting an end has the effect of scaling the eigenvalue appearing in the asymptotic formula (once the formula has been rewritten so that the first component does not contain an $\mathbb{R}$-shift). Consequently, we get either the strict inequality

$$
\begin{aligned}
i^{\Phi}\left([\tilde{u} ; z],\left[\tilde{v}_{c} ; w\right]\right) & >-m n \max \left\{\frac{\operatorname{wind}_{\infty}^{\Phi}(\tilde{u} ; z)}{|m|}, \frac{\operatorname{wind}_{\infty}^{\Phi}\left(\tilde{v}_{c} ; w\right)}{|n|}\right\} \\
& =-m n \max \left\{\frac{\operatorname{wind}_{\infty}^{\Phi}(\tilde{u} ; z)}{|m|}, \frac{\operatorname{wind}_{\infty}^{\Phi}(\tilde{v} ; w)}{|n|}\right\}
\end{aligned}
$$

or that $i^{\Phi}\left([\tilde{u} ; z],\left[\tilde{v}_{c} ; w\right]\right)$ is not defined for at most one value of $c \in \mathbb{R}$. For all other values we will have

$$
i^{\Phi}\left([\tilde{u} ; z],\left[\tilde{v}_{c} ; w\right]\right)=-m n \max \left\{\frac{\operatorname{wind}_{\infty}^{\Phi}(\tilde{u} ; z)}{|m|}, \frac{\operatorname{wind}_{\infty}^{\Phi}(\tilde{v} ; w)}{|n|}\right\} .
$$

Adding $m n \max \left\{\frac{\alpha\left(\gamma^{m}\right)}{|m|}, \frac{\alpha\left(\gamma^{n}\right)}{|n|}\right\}$ to both sides of this, we can conclude that the asymptotic intersection number of the two ends satisfies

$$
\delta_{\infty}([\tilde{u} ; z],[\tilde{v} ; w]) \geq m n\left(\max \left\{\frac{\alpha\left(\gamma^{m}\right)}{|m|}, \frac{\alpha\left(\gamma^{n}\right)}{|n|}\right\}-\max \left\{\frac{\operatorname{wind}_{\infty}^{\Phi}(\tilde{u} ; z)}{|m|}, \frac{\operatorname{wind}_{\infty}^{\Phi}(\tilde{v} ; w)}{|n|}\right\}\right)
$$

with the strict inequality occurring for at most one value of $c \in \mathbb{R}$.

The claims of the Lemma now follow from applying the results of the previous paragraph pairwise to ends of $\tilde{u}$ and $\tilde{v}_{c}$ which are asymptotic to coverings of the same orbit with the same sign, together with (4-5).

As a corollary of Theorem 5.8 and Lemma 5.10 we have the following.

Corollary 5.11 Let $[\Sigma, j, \Gamma, \tilde{u}=(a, u)],\left[\Sigma^{\prime}, j^{\prime}, \Gamma^{\prime}, \tilde{v}=(b, v)\right] \in \mathcal{M}(M, \mathcal{H}, J)$ be pseudoholomorphic curves and let $\tilde{v}_{c}=(b+c, v)$ be the curve resulting from translating $\tilde{v}$ in the $\mathbb{R}$ coordinate by $c$. Assume that no component of $\tilde{u}$ or $\tilde{v}$ has image contained in an orbit cylinder and let $\gamma_{z}^{m_{z}}$ and $\gamma_{w}^{m_{w}}$ denote the asymptotic limits of $\tilde{u}$ at $z \in \Gamma$ 
and $\tilde{v}$ at $w \in \Gamma^{\prime}$ as in Theorem 5.8. Then the algebraic intersection number $\operatorname{int}\left(\tilde{u}, \tilde{v}_{c}\right)$ is defined for all but a finite number of values of $c \in \mathbb{R}$. Moreover, with $\bar{\Lambda}$ as defined in (5-6), we have that

$$
\begin{aligned}
\operatorname{int}\left(\tilde{u}, \tilde{v}_{c}\right) \leq & \sum_{w \in \Gamma_{+}^{\prime}}\left|m_{w}\right|\left(\operatorname{int}\left(\gamma_{w}, u\right)+\sum_{\substack{z \in \Gamma_{+} \\
\gamma_{z}=\gamma_{w}}}\left|m_{z}\right| \bar{\Lambda}\left(\frac{\operatorname{wind}_{\infty}(\tilde{v} ; w)}{\left|m_{w}\right|}, \frac{\operatorname{wind}_{\infty}(\tilde{u} ; z)}{\left|m_{z}\right|}\right)\right) \\
(5-10) \quad & +\sum_{z \in \Gamma_{-}}\left|m_{z}\right|\left(\operatorname{int}\left(\gamma_{z}, v\right)+\sum_{\substack{w \in \Gamma_{\bar{\gamma}}^{\prime} \\
\gamma_{w}=\gamma_{z}}}\left|m_{w}\right| \bar{\Lambda}\left(\frac{\operatorname{wind}_{\infty}(\tilde{u} ; z)}{\left|m_{z}\right|}, \frac{\operatorname{wind}_{\infty}(\tilde{v} ; w)}{\left|m_{w}\right|}\right)\right) \\
& +\sum_{(z, w) \in \Gamma_{-} \times \Gamma_{+}^{\prime}}\left|m_{w}\right| d_{0}(\tilde{u} ; z)+\left|m_{z}\right| d_{0}(\tilde{v} ; w)-\left|m_{w} m_{z}\right|\left[\frac{\alpha\left(\gamma_{z}^{m_{z}}\right)}{\left|m_{z}\right|}+\frac{\alpha\left(\gamma_{w}^{m}\right)}{\left|m_{w}\right|}\right]
\end{aligned}
$$

and strict inequality occurs for at most a finite set of values of $c \in \mathbb{R}$ for which int $\left(\tilde{u}, \tilde{v}_{c}\right)$ is defined.

Proof This follows from subtracting

$$
\sum_{\substack{(z, w) \in \Gamma \times \Gamma^{\prime} \\ \gamma_{z}=\gamma_{w} \\ m_{z} m_{w}>0}} m_{z} m_{w}\left(\max \left\{\frac{\alpha(\tilde{u} ; z)}{\left|m_{z}\right|}, \frac{\alpha(\tilde{v} ; w)}{\left|m_{w}\right|}\right\}-\max \left\{\frac{\operatorname{wind}_{\infty}(\tilde{u} ; z)}{\left|m_{z}\right|}, \frac{\operatorname{wind}_{\infty}(\tilde{v} ; w)}{\left|m_{w}\right|}\right\}\right)
$$

from (5-5) and using Lemma 5.10.

We can now prove Theorem 2.4, which gives necessary and sufficient conditions for two projected curves to not intersect. We restate the result here for the convenience of the reader.

Theorem 5.12 (Theorem 2.4) Let $[\Sigma, j, \Gamma, \tilde{u}=(a, u)]$ and $\left[\Sigma^{\prime}, j^{\prime}, \Gamma^{\prime}, \tilde{v}=(b, v)\right] \in$ $\mathcal{M}(M, \mathcal{H}, J)$ be pseudoholomorphic curves and assume that no component of $\tilde{u}$ or $\tilde{v}$ lies in in orbit cylinder and that the projected curves $u$ and $v$ do not have identical image on any component of their domains. Then the following are equivalent:

(1) The projected curves $u$ and $v$ do not intersect.

(2) All of the following hold:

(a) The map $u$ does not intersect any of the positive asymptotic limits of $v$.

(b) The map $v$ does not intersect any of the negative asymptotic limits of $u$.

(c) If $\gamma$ is a periodic orbit so that at $z \in \Gamma, \tilde{u}$ is asymptotic to $\gamma^{m_{z}}$ and at $w \in \Gamma^{\prime}, \tilde{v}$ is asymptotic to $\gamma^{m_{w}}$, then: 
(i) If $m_{z}$ and $m_{w}$ have the same sign then

$$
\frac{\operatorname{wind}_{\infty}(\tilde{u} ; z)}{m_{z}} \geq \frac{\operatorname{wind}_{\infty}(\tilde{v} ; w)}{m_{w}} .
$$

(ii) If $m_{z}<0$ and $m_{w}>0$ then $d_{0}(\tilde{u} ; z)=d_{0}(\tilde{v} ; w)=0$ and $\gamma^{m_{z}}$ and $\gamma^{m_{w}}$ are both even orbits; or equivalently

$$
\frac{\operatorname{wind}_{\infty}(\tilde{u} ; z)}{m_{z}}=\frac{\operatorname{wind}_{\infty}(\tilde{v} ; w)}{m_{w}} .
$$

(3) All of the following hold:

(a) The map $u$ does not intersect any of the asymptotic limits of $v$.

(b) The map $v$ does not intersect any of the asymptotic limits of $u$.

(c) If $\gamma$ is a periodic orbit so that at $z \in \Gamma, \tilde{u}$ is asymptotic to $\gamma^{m_{z}}$ and at $w \in \Gamma^{\prime}, \tilde{v}$ is asymptotic to $\gamma^{m_{w}}$, then

$$
\frac{\operatorname{wind}_{\infty}(\tilde{u} ; z)}{m_{z}}=\frac{\operatorname{wind}_{\infty}(\tilde{v} ; w)}{m_{w}} .
$$

Proof The fact that (1) $\Longleftrightarrow(2)$ follows from an argument similar to that in the proof of Corollary 5.9 using now (5-10) instead of (5-5). Indeed, we have that the projected curves $u$ and $v$ are disjoint if and only if $\tilde{u}$ and $\tilde{v}_{c}$ are disjoint for all $c \in \mathbb{R}$, which by positivity of intersections is true precisely when $\operatorname{int}\left(\tilde{u}, \tilde{v}_{c}\right)=0$ for all $c \in \mathbb{R}$. Again, by positivity of intersections and Corollary 5.11 it is necessary and sufficient for the right hand side of (5-10) to vanish. Since the right hand side of (5-10) decomposes into nonnegative terms, we need to require each of these terms to vanish. We can argue as in Corollary 5.9 that the vanishing of each of these terms corresponds to the conditions listed in (2) above. Therefore (1) $\Longleftrightarrow(2)$ as claimed.

The fact that $(2) \Longleftrightarrow(3)$, like in Corollary 5.9, follows from the symmetry of the intersection number and the asymmetry of statement (2). Indeed, if (2) holds for $\tilde{u}$ and $\tilde{v}$ as stated, then (2) holds with the roles of $\tilde{u}$ and $\tilde{v}$ reversed. Thus (2) holds precisely when:

- $\operatorname{int}\left(\gamma_{w}, u\right)=0$ for all $w \in \Gamma^{\prime}$,

- $\operatorname{int}\left(\gamma_{z}, v\right)=0$ for all $z \in \Gamma$,

- for every $(z, w) \in \Gamma \times \Gamma^{\prime}$ with $\gamma_{z}=\gamma_{w}$ and $m_{z} m_{w}>0$

$$
\frac{\operatorname{wind}_{\infty}(\tilde{u} ; z)}{m_{z}}=\frac{\operatorname{wind}_{\infty}(\tilde{v} ; w)}{m_{w}},
$$

and

- for every $(z, w) \in \Gamma \times \Gamma^{\prime}$ with $\gamma_{z}=\gamma_{w}$ and $m_{z} m_{w}<0$

$$
\frac{\operatorname{wind}_{\infty}(\tilde{u} ; z)}{m_{z}}=\frac{\operatorname{wind}_{\infty}(\tilde{v} ; w)}{m_{w}} .
$$


These conditions are equivalent to those in statement (3) and thus (2) $\Longleftrightarrow(3)$ as claimed.

\subsubsection{Positive asymptotic intersection indices and direction of approach to even} orbits In this section we give a sufficient condition for positivity of the generalized intersection number of two curves in terms of direction of approach to shared hyperbolic orbits.

Before stating and proving the result, we will need to establish some terminology. Let $\gamma$ be a periodic orbit of $X_{\mathcal{H}}$ and assume that either

- $\gamma$ is a simple even orbit or

- $\gamma=\hat{\gamma}^{2}$ where $\hat{\gamma}$ is a simple, odd, hyperbolic orbit.

In either of these cases, let $\lambda^{-}=\sigma_{\max }^{-}(\gamma) \in \sigma\left(\mathbf{A}_{\gamma, J}\right)$ be the largest negative eigenvalue of $\mathbf{A}_{\gamma, J}$.

Lemma 5.13 With $\gamma, \lambda^{-}$satisfying the assumptions above, $m \lambda^{-}$is the largest negative eigenvalue of $\mathbf{A}_{\gamma^{m}, J}$ for any positive integer $m$. Moreover, the eigenspace

$$
\operatorname{ker}\left(\mathbf{A}_{\gamma^{m}, J}-m \lambda^{-}\right)
$$

is one dimensional for all positive integers $m$ and, if $e^{-} \in \operatorname{ker}\left(\mathbf{A}_{\gamma, J}-\lambda^{-}\right)$is a basis, then $e_{m}^{-}:=e^{-}(m \cdot)$ is a basis for $\operatorname{ker}\left(\mathbf{A}_{\gamma^{m}, J}-m \lambda^{-}\right)$.

Proof By the assumption that $\gamma$ is an even orbit and the definition of parity (3-3), there exists a $\lambda^{+}>0$ so that eigenvectors with eigenvalue $\lambda^{-}$and eigenvectors with eigenvalue $\lambda^{+}$have the same winding number in any choice of trivialization of $\gamma^{*} \xi^{\mathcal{H}}$. Therefore, by Lemma 3.1, we know that

and

$$
\operatorname{ker}\left(\mathbf{A}_{\gamma, J}-\lambda^{-}\right)
$$

are both 1-dimensional.

Let $e^{ \pm}$be a basis for $\operatorname{ker}\left(\mathbf{A}_{\gamma, J}-\lambda^{ \pm}\right)$. By the discussion following Lemma 3.1, we know that $e_{m}^{ \pm}$defined by $e_{m}^{ \pm}(t)=e^{ \pm}(m t)$ are eigenvectors with eigenvalue $m \lambda^{ \pm}$. Moreover, $e_{m}^{+}$and $e_{m}^{-}$will have the same winding in any trivialization, so it follows from Lemma 3.1 that $e_{m}^{ \pm}$is a basis for $\operatorname{ker}\left(\mathbf{A}_{\gamma^{m}, J}-m \lambda^{ \pm}\right)$and, since winding is monotonic in eigenvalue, we know that $m \lambda^{-}$must be the largest negative eigenvalue of $\mathbf{A}_{\gamma^{m}, J}$. 
Now, with $\gamma$ as above, consider a pseudoholomorphic end $[\Sigma, j, z, \tilde{u}]$ which is asymptotic to $\gamma^{m}$ for some positive integer $m$. Let $(U, \phi)$ be an asymptotic representation of $u$ near $z$, so that

$$
\tilde{u}(\phi(s, t))=\left(m \tau s, \exp _{\gamma^{m}(t)} U(s, t)\right) .
$$

Then, it follows from a special case of Theorem 3.7 that there exists a (possibly zero) $e_{u} \in \operatorname{ker}\left(\mathbf{A}_{\gamma, J}-\lambda^{-}\right)$satisfying

$$
e^{-m \lambda^{-} s}\left[U(s, t)-e_{u}(m t)\right] \rightarrow 0
$$

as $s \rightarrow \infty$. Considering a second end $\left[\Sigma^{\prime}, j^{\prime}, z^{\prime}, \tilde{v}\right]$ asymptotic to $\gamma^{n}$ with $n>0$, we find an asymptotic representative $(V, \psi)$ and a vector $e_{v} \in \operatorname{ker}\left(\mathbf{A}_{\gamma, J}-\lambda^{-}\right)$so that we can write

$$
\tilde{v}(\psi(s, t))=\left(n \tau s, \exp _{\gamma^{n}(t)} V(s, t)\right)
$$

with $V$ satisfying

$$
e^{-n \lambda^{-} s}\left[V(s, t)-e_{v}(n t)\right] \rightarrow 0
$$

as $s \rightarrow \infty$. If there exists a positive real number $c$ so that

$$
e_{u}=c e_{v}
$$

then we say that the ends $[\Sigma, j, z, \tilde{u}]$ and $\left[\Sigma^{\prime}, j^{\prime}, z^{\prime}, \tilde{v}\right]$ approach coverings of $\gamma$ in the same direction. Note that in the case that one of $e_{u}$ or $e_{v}$ is zero, this condition would imply that both of $e_{u}$ and $e_{v}$ are zero.

For simplicity in this discussion, we have only considered ends with positive punctures, but we can analogously define what it means for ends with negative punctures to approach a covering of $\gamma$ in the same direction by describing the direction of approach with elements of $\operatorname{ker}\left(\mathbf{A}_{\gamma_{-}, J}-\hat{\lambda}^{-}\right)$where $\gamma_{-}=\gamma^{(-1)}$ is $\gamma$ traversed backwards and $\hat{\lambda}^{-}$is the largest negative eigenvalue of $\mathbf{A}_{\gamma_{-}, J} \cdot{ }^{9}$

Theorem 5.14 Let $\gamma$ be an even periodic orbit satisfying the assumptions above, let $[\Sigma, j, z, \tilde{u}]$ and $\left[\Sigma^{\prime}, j^{\prime}, z^{\prime}, \tilde{v}\right]$ be distinct (nonintersecting) pseudoholomorphic ends in $\mathbb{R} \times M$ satisfying the assumptions above and let $e_{u}, e_{v} \in \operatorname{ker}\left(\mathbf{A}_{\gamma, J}-\lambda^{-}\right)$be defined as above. If $e_{u}=e_{v}$, then the asymptotic intersection index $\delta_{\infty}([\tilde{u} ; z],[\tilde{v} ; w])$ of the two ends is positive.

${ }^{9}$ Alternatively, we could use the fact that if $[\Sigma, j, z,(a, u)]$ is an end with a positive puncture in $\mathbb{R} \times M$ where $M$ is equipped with the Hamiltonian structure $(\lambda, \omega))$, then $[\Sigma, j, z,(-a, u)]$ is an end with a negative puncture in $\mathbb{R} \times M$ where $M$ is now equipped with the Hamiltonian structure $(-\lambda, \omega)$. This approach is employed in some proofs in [14]. 
Proof For simplicity we assume $m$ and $n$ are both positive, but the proof readily adapts to the case where $m$ and $n$ are both negative.

Letting $e_{u}$ and $e_{v}$ be as above, we first assume that $e_{u}=e_{v}=0$. Then, it follows that the eigenvalue of the leading eigenvector in the formulas for $\tilde{u}$ from Theorem 3.7 is strictly less than $m \lambda^{-}$and similarly the eigenvalue for the leading term in the formula for $\tilde{v}$ is strictly less than $n \lambda^{-}$. Since $\gamma$ is an even orbit, Lemma 3.1 and (3-3) imply that

$$
\begin{aligned}
& \operatorname{wind}_{\infty}^{\Phi}(\tilde{u} ; z)<\alpha^{\Phi}\left(\gamma^{m}\right)=m \alpha^{\Phi}(\gamma) \\
& \operatorname{wind}_{\infty}^{\Phi}(\tilde{v} ; w)<\alpha^{\Phi}\left(\gamma^{n}\right)=n \alpha^{\Phi}(\gamma)
\end{aligned}
$$

where we have used Lemma 5.13 to conclude that $\alpha^{\Phi}\left(\gamma^{k}\right)=k \alpha^{\Phi}(\gamma)$ for any positive integer $k$. In the case that $\gamma$ is a simply covered even orbit, we consequently get from (3-30) and (5-13) that

$$
\begin{aligned}
i_{\infty}^{\Phi}([\tilde{u} ; z],[\tilde{v} ; w]) & \geq-m n \max \left\{\frac{\operatorname{wind}_{\infty}^{\Phi}(\tilde{u} ; z)}{m}, \frac{\operatorname{wind}_{\infty}^{\Phi}(\tilde{v} ; w)}{n}\right\} \\
& >-m n \alpha^{\Phi}(\gamma) \\
& =-m n \max \left\{\frac{\alpha^{\Phi}\left(\gamma^{m}\right)}{m}, \frac{\alpha^{\Phi}\left(\gamma^{n}\right)}{n}\right\}
\end{aligned}
$$

and so

$$
\delta_{\infty}([\tilde{u} ; z],[\tilde{v} ; w])=i_{\infty}^{\Phi}([\tilde{u} ; z],[\tilde{v} ; w])+m n \max \left\{\frac{\alpha^{\Phi}\left(\gamma^{m}\right)}{m}, \frac{\alpha^{\Phi}\left(\gamma^{n}\right)}{n}\right\}>0
$$

as claimed. If $\gamma=\hat{\gamma}^{2}$ with $\hat{\gamma}$ a simple, odd, hyperbolic orbit, we have from (3-6) that

$$
\alpha(\gamma)=\alpha\left(\hat{\gamma}^{2}\right)=2 \alpha(\hat{\gamma})+1
$$

Consequently we find, again using (3-30) and (5-13), that

$$
\begin{aligned}
i_{\infty}^{\Phi}([\tilde{u} ; z],[\tilde{v} ; w]) & \geq-(2 m)(2 n) \max \left\{\frac{\operatorname{wind}_{\infty}^{\Phi}(\tilde{u} ; z)}{2 m}, \frac{\operatorname{wind}_{\infty}^{\Phi}(\tilde{v} ; w)}{2 n}\right\} \\
& >-2 m n\left(\alpha^{\Phi}(\gamma)\right) \\
& =-2 m n\left(2 \alpha^{\Phi}(\hat{\gamma})+1\right) \\
& =-(2 m)(2 n) \max \left\{\frac{\alpha^{\Phi}\left(\hat{\gamma}^{2 m}\right)}{2 m}, \frac{\hat{\alpha}^{\Phi}\left(\hat{\gamma}^{2 n}\right)}{2 n}\right\},
\end{aligned}
$$

where in the last line we have used

$$
\alpha^{\Phi}\left(\hat{\gamma}^{2 k}\right)=k(2 \alpha(\hat{\gamma})+1)
$$


from (3-6). Again we conclude that

$$
\delta_{\infty}([\tilde{u} ; z],[\tilde{v} ; w])=i_{\infty}^{\Phi}([\tilde{u} ; z],[\tilde{v} ; w])+(2 m)(2 n) \max \left\{\frac{\alpha^{\Phi}\left(\hat{\gamma}^{2 m}\right)}{2 m}, \frac{\alpha^{\Phi}\left(\hat{\gamma}^{2 n}\right)}{2 n}\right\}>0
$$

as claimed.

Next we assume that $e_{u}=e_{v} \neq 0$. With $(U, \phi)$ and $(V, \psi)$ satisfying (5-11) and (5-12) respectively, we can apply Theorem 3.6 to write

$$
U(n s, n t)-V(m s, m t)=e^{\mu s}[e(t)+r(s, t)]
$$

with $\mu<0$ an eigenvalue of $\mathbf{A}_{\gamma^{m n}, J}, e$ and eigenvector with eigenvalue $\mu$ and $r$ converging exponentially to zero as $s \rightarrow \infty$. The assumption that $e_{u}=e_{v}$ then implies that $\mu<m n \lambda^{-}$, which, since $\gamma^{m n}$ is an even orbit, implies that

$$
\text { wind } \Phi^{-1} e<\text { wind } \Phi^{-1} e_{u}(m n \cdot)=m n \text { wind } \Phi^{-1} e_{u}=m n \alpha^{\Phi}(\gamma) \text {. }
$$

We can therefore conclude that

$$
\operatorname{wind}_{\mathrm{rel}}^{\Phi}(n \cdot[\tilde{u} ; z], m \cdot[\tilde{v} ; w])<m^{2} n^{2} \alpha^{\Phi}(\gamma)
$$

which by Lemma 3.20 implies that

$$
i_{\infty}^{\Phi}([\tilde{u} ; z],[\tilde{v} ; w])>-m n \alpha^{\Phi}(\gamma) .
$$

Since $\gamma$ is assumed to be either a simple even orbit or a double covered odd hyperbolic orbit, it follows as in the previous paragraph that

$$
\delta_{\infty}([\tilde{u} ; z],[\tilde{v} ; w])>0
$$

in either case.

We now prove the main result of this section.

Theorem 5.15 (Theorem 2.5) Let $\gamma$ be a periodic orbit satisfying the above assumptions and let $[\Sigma, j, \Gamma, \tilde{u}=(a, u)]$ and $\left[\Sigma^{\prime}, j^{\prime}, \Gamma^{\prime}, \tilde{v}=(b, v)\right] \in \mathcal{M}(M, \mathcal{H}, J)$ be connected pseudoholomorphic curves. If there are punctures $z \in \Gamma$ and $w \in \Gamma^{\prime}$ so that the ends $[\tilde{u} ; z]$ and $[\tilde{v} ; w]$ approach a cover of $\gamma$ in the same direction and so that $[\tilde{u} ; z]$ is distinct from the $\mathbb{R}$-shifted end $[(b+c, v) ; w]$ for all $c \in \mathbb{R}$ then

$$
[\tilde{u}] *[\tilde{v}]>0 \text {. }
$$

Proof For simplicity we assume both punctures $z$ and $w$ are positive, but the argument readily adapts to the case where they are both negative. 
Let $(U, \phi)$ and $(V, \phi)$ satisfy (5-11) and (5-12) and let $e_{u}$ and $e_{v}$ be as defined above. We first consider the case that $e_{u}=e_{v}=0$. In this case, the lemma above shows that the asymptotic intersection index $\delta_{\infty}([\tilde{u} ; z],[\tilde{v} ; w])$ is positive and thus the total asymptotic intersection index $\delta_{\infty}(\tilde{u}, \tilde{v})$ is positive. Thus, Theorem 4.4 gives us

$$
[\tilde{u}] *[\tilde{v}]=\operatorname{int}(\tilde{u}, \tilde{v})+\delta_{\infty}(\tilde{u}, \tilde{v}) \geq \delta_{\infty}(\tilde{u}, \tilde{v})>0
$$

as claimed.

In the case that $e_{u}=c e_{v} \neq 0$ for some positive real number $c$, we apply the observation from the proof of Lemma 5.10 that $\mathbb{R}$-shifting a curve has the effect of scaling the eigenvector describing the approach. Indeed, if we let $\tilde{v}_{c^{\prime}}$ denote the map

$$
\tilde{v}_{c^{\prime}}=\left(b+c^{\prime}, v\right)
$$

and let $\left(V_{c^{\prime}}, \psi_{c^{\prime}}\right)$ denote an asymptotic representative of $\tilde{v}_{c^{\prime}}$, we can choose a $c^{\prime}$ so that $e_{v_{c^{\prime}}}$, chosen to satisfy

$$
e^{-n \lambda^{-} s}\left(V_{c^{\prime}}(s, t)-e_{v_{c^{\prime}}}(n t)\right) \rightarrow 0 \text { as } s \rightarrow \infty,
$$

is equal to $e_{u}$. The previous lemma then applies to show that $\delta_{\infty}\left([\tilde{u} ; z],\left[\tilde{v}_{c^{\prime}}, w\right]\right)>0$ and hence, by the homotopy invariance of the generalized intersection number, that

$$
[\tilde{u}] *[\tilde{v}]=[\tilde{u}] *\left[\tilde{v}_{c^{\prime}}\right] \geq \delta_{\infty}\left([\tilde{u} ; z],\left[\tilde{v}_{c^{\prime}}, w\right]\right)>0
$$

as claimed.

\subsection{Generalized self-intersection numbers and embeddedness of the pro- jection}

In this section we investigate conditions under which a curve in a cylindrical cobordism has generalized self-intersection number equal to zero and we investigate controls on the embeddedness of the projection of a pseudoholomorphic curve in $\mathbb{R} \times M$ into the three manifold $M$. The key observation for this latter question, originally made by Hofer, Wysocki and Zehnder in [8], is that the projected curve is injective if and only if the nonprojected curve does not intersect any of its $\mathbb{R}$-translates.

We first state Theorem 5.8 in the special case that $\tilde{u}=\tilde{v}$.

Corollary 5.16 Let $(\Sigma, j, \Gamma, \tilde{u}=(a, u)) \in C^{\infty}(M, \mathcal{H})$ be an asymptotically cylindrical map with ends that wind and assume that at $z \in \Gamma, \tilde{u}$ is asymptotic to $\gamma_{z}^{m_{z}}$, with 
$\gamma_{z} \in \mathcal{P}^{0}(M, \mathcal{H})$ a simple periodic orbit and $m_{z}$ a nonzero integer. Then

$$
\begin{aligned}
{[\tilde{u}] *[\tilde{u}]=} & \sum_{z \in \Gamma}\left|m_{z}\right|\left(\operatorname{int}\left(\gamma_{z}, u\right)+\sum_{\substack{w \in \Gamma \\
\gamma_{w}=\gamma_{z} \\
m_{z} m_{w}>0}}\left|m_{w}\right| \bar{\Lambda}\left(\frac{\alpha\left(\gamma_{z}^{m_{z}}\right)}{\left|m_{z}\right|}, \frac{\alpha\left(\gamma_{w}^{m_{w}}\right)}{\left|m_{w}\right|}\right)+d_{0}(\tilde{u} ; w)\right) \\
& +\sum_{\substack{(z, w) \in \Gamma_{+} \times \Gamma_{-} \\
\gamma_{z}=\gamma_{w}}}\left|m_{w}\right| d_{0}(\tilde{u} ; z)+\left|m_{z}\right| d_{0}(\tilde{u} ; w)-\left|m_{z} m_{w}\right|\left[\frac{\alpha\left(\gamma_{z}^{m_{z}}\right)}{\left|m_{z}\right|}+\frac{\alpha\left(\gamma_{w}^{m}\right)}{\left|m_{w}\right|}\right]
\end{aligned}
$$

with $\bar{\Lambda}$ as defined in (5-6).

As a corollary to this and Corollary 5.1 we prove the following result which gives a set of equivalent conditions to $[\tilde{u}] *[\tilde{u}]=0$ for a simple curve.

Corollary 5.17 Let $[\Sigma, j, \Gamma, \tilde{u}=(a, u)] \in \mathcal{M}(M, \mathcal{H}, J)$ be a simple pseudoholomorphic curve and assume that no component of $\tilde{u}$ lies in an orbit cylinder. Then the following are equivalent:

(1) The generalized self-intersection number of $\tilde{u}$ vanishes, ie $[\tilde{u}] *[\tilde{u}]=0$

(2) All of the following hold:

(a) The projected map $u$ does not intersect any of its asymptotic limits.

(b) For all $z \in \Gamma, d_{0}(\tilde{u} ; z)=0$.

(c) If $\gamma$ is a periodic orbit so that at $z \in \Gamma, \tilde{u}$ is asymptotic to $\gamma^{m_{z}}$ and at $w \in \Gamma$, $\tilde{u}$ is asymptotic to $\gamma^{m_{w}}$, then

$$
\frac{\alpha\left(\gamma^{m_{z}}\right)}{m_{z}}=\frac{\alpha\left(\gamma^{m_{w}}\right)}{m_{w}} .
$$

(3) All of the following hold:

(a) $\operatorname{ind}(\tilde{u})-\chi(\Sigma)+\# \Gamma_{\text {even }}=0$

(b) $\bar{\sigma}(\tilde{u} ; z)=1$ for all $z \in \Gamma$.

(c) $\operatorname{sing}(\tilde{u})=0$ or equivalently, $\tilde{u}$ is embedded and has total asymptotic selfintersection index, $\delta_{\infty}(\tilde{u})$, equal to zero.

(4) All of the following hold:

(a) The map $u$ is an immersion that is everywhere transverse to $X_{\mathcal{H}}$.

(b) $d_{0}(\tilde{u} ; z)=0$ for all $z \in \Gamma$.

(c) $\bar{\sigma}(\tilde{u} ; z)=1$ for all $z \in \Gamma$.

(d) $\operatorname{sing}(\tilde{u})=0$ or equivalently, $\tilde{u}$ is embedded and has total asymptotic selfintersection index, $\delta_{\infty}(\tilde{u})$, equal to zero. 
Proof The fact that $(1) \Longleftrightarrow(2)$ follows from Corollary 5.16 and an argument very similar to that in Corollary 5.9. We omit the details.

The fact that (1) $\Longleftrightarrow$ (4) follows from rewriting (5-2) as

$$
\begin{aligned}
{[\tilde{u}] *[\tilde{u}] } & =2 \operatorname{sing}(\tilde{u})+\operatorname{wind}_{\pi}(\tilde{u})+d_{0}(\tilde{u})+\bar{\sigma}(\tilde{u})-\# \Gamma \\
& =2 \operatorname{sing}(\tilde{u})+\operatorname{wind}_{\pi}(\tilde{u})+\sum_{z \in \Gamma}\left(d_{0}(\tilde{u} ; z)+[\bar{\sigma}(\tilde{u} ; z)-1]\right)
\end{aligned}
$$

and noting that each term on the right hand side of this is nonnegative.

Finally, the fact that (4) $\Longleftrightarrow$ (3) follows directly from (5-1). Note that (5-1) implies that the quantity ind $(\tilde{u})-\chi(\Sigma)+\# \Gamma_{\text {even }}$ is nonnegative for curves in a cylindrical cobordism provided it has no components with image contained in an orbit cylinder.

We observe that for a connected curve $[\Sigma, j, \Gamma, \tilde{u}]$ satisfying the hypotheses of the previous result, if $[\tilde{u}] *[\tilde{u}]=0$ then the projected curve $u$ is an embedding in the threemanifold. Indeed, the result shows that $u$ must be an immersion which does not intersect any of its asymptotic limits. Moreover, since for the $\mathbb{R}$-translates $\tilde{u}_{c}=(a+c, u)$, we have

$$
0 \leq \operatorname{int}\left(\tilde{u}, \tilde{u}_{c}\right) \leq[\tilde{u}] *\left[\tilde{u}_{c}\right]=[\tilde{u}] *[\tilde{u}]=0,
$$

it follows from positivity of intersections that $\tilde{u}$ does not intersect any of its $\mathbb{R}$-translates and hence that the projection $u$ is injective. As observed in [8], the asymptotic behavior of $u$ then allows us to conclude that $u$ is an embedding. As with the discussion of intersections of curves with distinct projections to the three-manifold, the converse is not true: it could well be the case that $u$ is an embedding and $[\tilde{u}] *[\tilde{u}] \neq 0$ since we could have that the total asymptotic intersection index $\delta_{\infty}\left(\tilde{u}, \tilde{u}_{c}\right)$ positive for all $c \in \mathbb{R} \backslash\{0\}$ for which it is defined. Again, in this case, since $\mathbb{R}$-shifting the curve changes the asymptotic intersection numbers in a predictable way, we can modify the above argument to find necessary and sufficient conditions for a curve to have an embedded projection.

Towards this end, we state the following special case of Corollary 5.11 when $\tilde{v}=\tilde{u}$.

Corollary 5.18 Let $[\Sigma, j, \Gamma, \tilde{u}=(a, u)] \in \mathcal{M}(M, \mathcal{H}, J)$ be a connected pseudoholomorphic curve and assume that the image of $\tilde{u}$ is not contained in an orbit cylinder. Then, with $\bar{\Lambda}$ as defined in (5-6), we have for any $c \in \mathbb{R} \backslash\{0\}$ that

$$
\operatorname{int}\left(\tilde{u}, \tilde{u}_{c}\right) \leq \sum_{w \in \Gamma}\left|m_{w}\right|\left(\operatorname{int}\left(\gamma_{w}, u\right)+\sum_{\substack{z \in \Gamma \\ \gamma_{z}=\gamma_{w} \\ m_{z} m_{w}>0}}\left|m_{z}\right| \bar{\Lambda}\left(\frac{\operatorname{wind}_{\infty}(\tilde{u} ; w)}{\left|m_{w}\right|}, \frac{\operatorname{wind}_{\infty}(\tilde{u} ; z)}{\left|m_{z}\right|}\right)\right)
$$




$$
+\sum_{\substack{(z, w) \in \Gamma_{-} \times \Gamma_{+} \\ \gamma_{z}=\gamma_{w}}}-\left|m_{z} m_{w}\right|\left(\frac{\operatorname{wind}_{\infty}(\tilde{u} ; z)}{\left|m_{z}\right|}+\frac{\operatorname{wind}_{\infty}(\tilde{u} ; w)}{\left|m_{w}\right|}\right)
$$

with strict inequality occurring for at most a finite number of values of $c \in \mathbb{R} \backslash\{0\}$.

Along similar lines, keeping track of how the asymptotic intersection numbers change with $\mathbb{R}$-shifts allows the following adjustment to Corollary 5.1

Lemma 5.19 Let $[\Sigma, j, \Gamma, \tilde{u}=(a, u)] \in \mathcal{M}(M, \mathcal{H}, J)$ be a connected, simple pseudoholomorphic curve and assume the image of $\tilde{u}$ is not contained in an orbit cylinder. Then, for any $c \in \mathbb{R} \backslash\{0\}$ we have that

$$
\begin{gathered}
\operatorname{int}\left(\tilde{u}, \tilde{u}_{c}\right) \leq \operatorname{wind}_{\pi}(\tilde{u})+2 \delta(\tilde{u})+\sum_{z_{i} \in \Gamma}\left[\operatorname{cov}\left(e_{1}\left(\tilde{u} ; z_{i}\right)\right)-1+\Delta_{2}\left(\tilde{u} ; z_{i}\right)\right] \\
+\sum_{\substack{z_{i}, z_{j} \in \Gamma \\
z_{i} \neq z_{j} \\
\gamma_{i}=\gamma_{z_{j}} \\
m_{z_{i}} m_{z_{j}}>0}} i_{\infty}\left(\left[\tilde{u} ; z_{i}\right],\left[\tilde{u} ; z_{j}\right]\right)+m_{z_{i}} m_{z_{j}} \max _{\ell=i, j}\left\{\frac{\operatorname{wind}_{\infty}\left(\tilde{u} ; z_{\ell}\right)}{\left|m_{z_{\ell}}\right|}\right\}
\end{gathered}
$$

where $e_{1}\left(\tilde{u} ; z_{i}\right)$ is as defined in (3-15), $\delta(u)$ is as defined in (4-10) and $\Delta_{2}\left(\tilde{u} ; z_{i}\right)$ is as defined in (3-22). Moreover equality occurs in (5-14) for all but a finite number of values of $c \in \mathbb{R} \backslash\{0\}$.

Proof By a special case of Corollary 5.11 we have that

$$
\begin{aligned}
& \operatorname{int}\left(\tilde{u}, \tilde{u}_{c}\right) \leq[\tilde{u}] *[\tilde{u}]-\sum_{\substack{z_{i}, z_{j} \in \Gamma \\
\gamma_{z_{i}}=\gamma_{z_{j}} \\
m_{z_{i}} m_{z_{j}}>0}} m_{z_{i}} m_{z_{j}}\left(\max _{\ell=i, j}\left\{\frac{\alpha\left(\tilde{u} ; z_{\ell}\right)}{\left|m_{z_{\ell}}\right|}\right\}-\max _{\ell=i, j}\left\{\frac{\operatorname{wind}\left(\tilde{u} ; z_{\ell}\right)}{\left|m_{z_{\ell}}\right|}\right\}\right) \\
&:=I(\tilde{u})
\end{aligned}
$$

with equality occurring for all but a finite number of values of $c \neq 0$. Applying the adjunction formula (5-2) we have that the right hand side of this inequality is equal to

$$
\begin{gathered}
I(\tilde{u})=\operatorname{wind}_{\pi}(\tilde{u})+d_{0}(\tilde{u})+[\bar{\sigma}(\tilde{u})-\# \Gamma]+2 \operatorname{sing}(\tilde{u}) \\
-\sum_{\substack{z_{i}, z_{j} \in \Gamma \\
\gamma_{z_{i}}=\gamma_{z_{j}} \\
m_{z_{i}} m_{z_{j}}>0}} m_{z_{i}} m_{z_{j}}\left(\max _{\ell=i, j}\left\{\frac{\alpha\left(\tilde{u} ; z_{\ell}\right)}{\left|m_{z_{\ell}}\right|}\right\}-\max _{\ell=i, j}\left\{\frac{\operatorname{wind}\left(\tilde{u} ; z_{\ell}\right)}{\left|m_{z_{\ell}}\right|}\right\}\right) .
\end{gathered}
$$


We rewrite the final sum in this by grouping the terms with $z_{i}=z_{j}$ and using the definition (3-18) of $d_{0}(\tilde{u} ; z)$ and (3-19) to get

$$
\begin{aligned}
& \sum_{\substack{z_{i}, z_{j} \in \Gamma \\
\gamma_{z_{i}}=\gamma_{z_{j}} \\
m_{z_{i}} m_{z_{j}}>0}} m_{z_{i}} m_{z_{j}}\left(\max _{\ell=i, j}\left\{\frac{\alpha\left(\tilde{u} ; z_{\ell}\right)}{\left|m_{z_{\ell}}\right|}\right\}-\max _{\ell=i, j}\left\{\frac{\operatorname{wind}\left(\tilde{u} ; z_{\ell}\right)}{\left|m_{z_{\ell}}\right|}\right\}\right) \\
& =\sum_{z_{i} \in \Gamma}\left|m_{z_{i}}\right| d_{0}\left(\tilde{u} ; z_{i}\right) \\
& +\sum_{z_{i}, z_{j} \in \Gamma} m_{z_{i}} m_{z_{j}}\left(\max _{\ell=i, j}\left\{\frac{\alpha\left(\tilde{u} ; z_{\ell}\right)}{\left|m_{z_{\ell}}\right|}\right\}-\max _{\ell=i, j}\left\{\frac{\operatorname{wind}\left(\tilde{u} ; z_{\ell}\right)}{\left|m_{z_{\ell}}\right|}\right\}\right) \\
& \begin{aligned}
z_{i} \neq z_{j} \\
\gamma_{z_{i}}=\gamma_{z_{j}}
\end{aligned} \\
& m_{z_{i}} m_{z_{j}}>0 \\
& =d_{0}(\tilde{u})+\sum_{z_{i} \in \Gamma} \Delta_{1}\left(\tilde{u} ; z_{i}\right)+\bar{\sigma}\left(\tilde{u} ; z_{i}\right)-\operatorname{cov}\left(e_{1}\left(\tilde{u} ; z_{i}\right)\right) \\
& +\sum_{z_{i}, z_{j} \in \Gamma} m_{z_{i}} m_{z_{j}}\left(\max _{\ell=i, j}\left\{\frac{\alpha\left(\tilde{u} ; z_{\ell}\right)}{\left|m_{z_{\ell}}\right|}\right\}-\max _{\ell=i, j}\left\{\frac{\operatorname{wind}\left(\tilde{u} ; z_{\ell}\right)}{\left|m_{z_{\ell}}\right|}\right\}\right) \\
& \begin{aligned}
z_{i} & \neq z_{j} \\
\gamma_{z_{i}} & =\gamma_{z_{j}}
\end{aligned} \\
& m_{z_{i}} m_{z_{j}}>0 \\
& =d_{0}(\tilde{u})+\bar{\sigma}(\tilde{u})-\# \Gamma+\sum_{z_{i} \in \Gamma} \Delta_{1}\left(\tilde{u} ; z_{i}\right)+1-\operatorname{cov}\left(e_{1}\left(\tilde{u} ; z_{i}\right)\right) \\
& +\sum_{z_{i}, z_{j} \in \Gamma} m_{z_{i}} m_{z_{j}}\left(\max _{\ell=i, j}\left\{\frac{\alpha\left(\tilde{u} ; z_{\ell}\right)}{\left|m_{z_{\ell}}\right|}\right\}-\max _{\ell=i, j}\left\{\frac{\operatorname{wind}\left(\tilde{u} ; z_{\ell}\right)}{\left|m_{z_{\ell}}\right|}\right\}\right), \\
& \begin{aligned}
z_{i} & \neq z_{j} \\
\gamma_{z_{i}} & =\gamma_{z_{j}}
\end{aligned} \\
& m_{z_{i}} m_{z_{j}}>0
\end{aligned}
$$

and we therefore have

$$
\begin{gathered}
I(\tilde{u})=\operatorname{wind}_{\pi}(\tilde{u})+2 \operatorname{sing}(\tilde{u})+\sum_{z_{i} \in \Gamma}\left[\operatorname{cov}\left(e_{1}\left(\tilde{u} ; z_{i}\right)\right)-1-\Delta_{1}\left(\tilde{u} ; z_{i}\right)\right] \\
-\sum_{\substack{z_{i}, z_{j} \in \Gamma \\
z_{i} \neq z_{j} \\
\gamma_{z_{i}}=\gamma_{z_{j}} \\
m_{z_{i}} m_{z_{j}}>0}} m_{z_{i}} m_{z_{j}}\left(\max _{\ell=i, j}\left\{\frac{\alpha\left(\tilde{u} ; z_{\ell}\right)}{\left|m_{z_{\ell}}\right|}\right\}-\max _{\ell=i, j}\left\{\frac{\operatorname{wind}\left(\tilde{u} ; z_{\ell}\right)}{\left|m_{z_{\ell}}\right|}\right\}\right) .
\end{gathered}
$$


Further, rewriting

$$
\begin{aligned}
2 \operatorname{sing}(\tilde{u})= & 2 \delta(\tilde{u})+2 \delta_{\infty}(\tilde{u}) \\
= & 2 \delta(\tilde{u})+\sum_{z_{i} \in \Gamma} 2 \delta_{\infty}\left(\tilde{u} ; z_{i}\right)+\sum_{\substack{z_{i}, z_{j} \in \Gamma \\
z_{i} \neq z_{j}}} \delta_{\infty}\left(\left[\tilde{u} ; z_{i}\right],\left[\tilde{u} ; z_{j}\right]\right) \\
= & 2 \delta(\tilde{u})+\sum_{z_{i} \in \Gamma} \Delta_{1}\left(\tilde{u} ; z_{i}\right)+\Delta_{2}\left(\tilde{u} ; z_{i}\right) \\
& +\sum_{\substack{z_{i}, z_{j} \in \Gamma \\
z_{i} \neq z_{j} \\
\gamma_{z_{i}}=\gamma_{z_{j}} \\
m_{z_{i}} m_{z_{j}}>0}} i_{\infty}\left(\left[\tilde{u} ; z_{i}\right],\left[\tilde{u} ; z_{j}\right]\right)+m_{z_{i}} m_{z_{j}} \max _{\ell=i, j}\left\{\frac{\alpha\left(\tilde{u} ; z_{\ell}\right)}{\left|m_{z_{\ell}}\right|}\right\} \\
= & 2 \delta(\tilde{u})+\sum_{\substack{z_{i} \in \Gamma \\
z_{i}}} \Delta_{1}\left(\tilde{u} ; z_{i}\right)+\Delta_{2}\left(\tilde{u} ; z_{i}\right) \\
& +\sum_{\substack{z_{i}, z_{j} \in \Gamma \\
z_{i} \neq z_{j} \\
\gamma_{z_{i}}=\gamma_{z_{j}} \\
m_{z_{i}} m_{z_{j}}>0}} i_{\infty}\left(\left[\tilde{u} ; z_{i}\right],\left[\tilde{u} ; z_{j}\right]\right)+m_{z_{i}} m_{z_{j}} \max _{\ell=i, j}\left\{\frac{\operatorname{wind}_{\infty}\left(\tilde{u} ; z_{\ell}\right)}{\left|m_{z_{\ell}}\right|}\right\} \\
& +\sum_{\substack{z_{i}, z_{j} \in \Gamma \\
z_{i} \neq z_{j} \\
\gamma_{z_{i}}=\gamma_{z_{j}} \\
m_{z_{i}} m_{z_{j}}>0}} m_{z_{i}} m_{z_{j}}\left(\max _{\ell=i, j}\left\{\frac{\alpha\left(\tilde{u} ; z_{\ell}\right)}{\left|m_{z_{\ell}}\right|}\right\}-\max _{\ell=i, j}\left\{\frac{\operatorname{wind}_{\infty}\left(\tilde{u} ; z_{\ell}\right)}{\left|m_{z_{\ell}}\right|}\right\}\right)
\end{aligned}
$$

allows us to write

$$
\begin{aligned}
& I(\tilde{u})=\operatorname{wind}_{\pi}(\tilde{u})+2 \delta(\tilde{u})+\sum_{z_{i} \in \Gamma}\left[\operatorname{cov}\left(e_{1}\left(\tilde{u} ; z_{i}\right)\right)-1+\Delta_{2}\left(\tilde{u} ; z_{i}\right)\right] \\
& +\sum_{\substack{z_{i}, z_{j} \in \Gamma \\
z_{i} \neq z_{j} \\
\gamma_{z_{i}}=\gamma_{z_{j}} \\
m_{z_{i}} m_{z_{j}}>0}} i_{\infty}\left(\left[\tilde{u} ; z_{i}\right],\left[\tilde{u} ; z_{j}\right]\right)+m_{z_{i}} m_{z_{j}} \max _{\ell=i, j}\left\{\frac{\operatorname{wind}_{\infty}\left(\tilde{u} ; z_{\ell}\right)}{\left|m_{z_{\ell}}\right|}\right\} .
\end{aligned}
$$

We therefore conclude that

$$
\operatorname{int}\left(\tilde{u}, \tilde{u}_{c}\right) \leq \operatorname{wind}_{\pi}(\tilde{u})+2 \delta(\tilde{u})+\sum_{z_{i} \in \Gamma}\left[\operatorname{cov}\left(e_{1}\left(\tilde{u} ; z_{i}\right)\right)-1+\Delta_{2}\left(\tilde{u} ; z_{i}\right)\right]
$$




$$
+\sum_{\substack{z_{i}, z_{j} \in \Gamma \\ z_{i} \neq z_{j} \\ \gamma_{z_{i}}=\gamma_{z_{j}} \\ m_{z_{i}} m_{z_{j}}>0}} i_{\infty}\left(\left[\tilde{u} ; z_{i}\right],\left[\tilde{u} ; z_{j}\right]\right)+m_{z_{i}} m_{z_{j}} \max _{\ell=i, j}\left\{\frac{\operatorname{wind}_{\infty}\left(\tilde{u} ; z_{\ell}\right)}{\left|m_{z_{\ell}}\right|}\right\} .
$$

with equality occurring for all but a finite number of values of $c \in \mathbb{R} \backslash\{0\}$.

We now prove Theorem 2.6 which gives necessary and sufficient conditions for the projection of a curve to the 3-manifold to be embedded.

Theorem 5.20 (Theorem 2.6) Let $[\Sigma, j, \Gamma, \tilde{u}=(a, u)] \in \mathcal{M}(M, \mathcal{H}, J)$ be a connected, simple pseudoholomorphic curve and assume that $\tilde{u}$ does not have image contained in an orbit cylinder. Then the following are equivalent:

(1) The projected map $u: \Sigma \backslash \Gamma \rightarrow M$ is an embedding.

(2) The intersection number $\operatorname{int}\left(\tilde{u}, \tilde{u}_{c}\right)$ between $\tilde{u}$ and an $\mathbb{R}$-translate $\tilde{u}_{c}=(a+c, u)$ is zero for all $c \in \mathbb{R} \backslash\{0\}$.

(3) All of the following hold:

(a) $u$ does not intersect any of its asymptotic limits.

(b) If $\gamma$ is a periodic orbit so that $u$ is asymptotic at $z \in \Gamma$ to $\gamma^{m_{z}}$ and $u$ is asymptotic at $w \in \Gamma$ to $\gamma^{m_{w}}$, then

$$
\frac{\operatorname{wind}_{\infty}(\tilde{u} ; z)}{m_{z}}=\frac{\operatorname{wind}_{\infty}(\tilde{u} ; w)}{m_{w}} .
$$

(4) All of the following hold:

(a) The map $\tilde{u}$ is an embedding.

(b) The projected map $u$ is an immersion which is everywhere transverse to $X_{\mathcal{H}}$

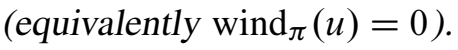

(c) For each $z \in \Gamma$, we have

$$
\operatorname{gcd}\left(m_{z}, \operatorname{wind}_{\infty}\left(\tilde{u} ; m_{z}\right)\right)=1
$$

(equivalently $\left.\operatorname{cov}\left(e_{1}(\tilde{u} ; z)\right)=1\right)$.

(d) If $\gamma$ is a periodic orbit so that $u$ is asymptotic at $z$ to $\gamma^{m_{z}}$ and $u$ is asymptotic at $w \neq z$ to $\gamma^{m_{w}}$ with $m_{z} m_{w}>0$, then the asymptotic intersection number of the ends $[\tilde{u} ; z]$ and $[\tilde{u} ; w]$ achieves the bound from (3-30), ie

$$
i_{\infty}^{\Phi}([\tilde{u} ; z],[\tilde{u} ; w])=-m_{z} m_{w} \max \left\{\frac{\operatorname{wind}_{\infty}^{\Phi}(\tilde{u} ; z)}{m_{z}}, \frac{\operatorname{wind}_{\infty}^{\Phi}(\tilde{u} ; w)}{m_{w}}\right\}
$$


Proof We first address $(2) \Longleftrightarrow(3)$. This follows from Corollary 5.18 and positivity of intersections by an argument analogous to that in Corollary 5.17. We omit the details. Next we prove $(2) \Longleftrightarrow(4)$. This is also similar to a part of Corollary 5.17. It follows from positivity of intersections and Lemma 5.19, particularly the nonnegativity of the each of the terms on the right hand side of (5-14). Indeed, from positivity of intersections and (5-14) we can conclude that $\operatorname{int}\left(\tilde{u}, \tilde{u}_{c}\right)=0$ for all $c \in \mathbb{R} \backslash\{0\}$ if and only if:

- $\operatorname{wind}_{\pi}(\tilde{u})=0$,

- $\delta(\tilde{u})=0$ (ie $\tilde{u}$ is an embedding),

- $\operatorname{cov}\left(e_{1}(\tilde{u} ; z)\right)=1$ for all $z \in \Gamma$,

- $\Delta_{2}(\tilde{u} ; z)=0$ for all $z \in \Gamma$ and

- the asymptotic intersection numbers satisfies

$$
i_{\infty}^{\Phi}([\tilde{u} ; z],[\tilde{u} ; w])=-m_{z} m_{w} \max \left\{\frac{\operatorname{wind}_{\infty}^{\Phi}(\tilde{u} ; z)}{m_{z}}, \frac{\operatorname{wind}_{\infty}^{\Phi}(\tilde{u} ; w)}{m_{w}}\right\}
$$

for each pair of distinct punctures $(z, w) \in \Gamma \times \Gamma$ at which $u$ is asymptotic to coverings of a the same underlying orbit with the same sign.

Recalling from Lemma 3.14 that $\operatorname{cov}\left(e_{1}(\tilde{u} ; z)\right)=1$ implies that $\Delta_{2}(\tilde{u} ; z)=0$ and from Lemma 3.2 and (3-16) that

$$
\operatorname{cov}\left(e_{1}(\tilde{u} ; z)\right)=\operatorname{gcd}\left(\operatorname{wind}\left(\Phi^{-1} e_{1}(\tilde{u} ; z)\right), m_{z}\right)=\operatorname{gcd}\left(\operatorname{wind}_{\infty}^{\Phi}(\tilde{u} ; z), m_{z}\right)
$$

we see that these conditions are equivalent to those listed in (4) above.

Next we observe that (1) $\Rightarrow(2)$. This has been previously observed by Hofer, Wysocki and Zehnder in [8], but we include the argument here for completeness, since it is short and illustrative. Indeed if there exists a $c \in \mathbb{R} \backslash\{0\}$ so that $\tilde{u}=(a, u)$ intersects $\tilde{u}_{c}=(a+c, u)$, then there is a pair of points distinct points $z, w \in \Sigma \backslash \Gamma$ so that $\tilde{u}(z)=\tilde{u}(w)$ which in turn implies that $u(z)=u(w)$. Thus (2) failing to hold implies that the projected map has a double point and hence cannot be an embedding.

Finally we show that (2), (3), (4) $\Rightarrow(1)$. Indeed if (2), (3) and (4) are all true, then the projected curve $u$ is an injective (by (2) and the argument of the previous paragraph) immersion (by (3)), which does not intersect any of its asymptotic limits (by (4)). As observed in [12], this with the asymptotic behavior implies that the map $u$ is an embedding. 


\subsection{Holomorphic open book decompositions}

In this section we examine an application of results from the previous sections to properties of holomorphic curves in manifolds admitting a holomorphic open book decomposition. Recall from the introduction $(M, \mathcal{H}, J)$ is said to admit a stable, holomorphic open book decomposition if there is a link $L \subset M$ made up of elliptic periodic orbits of $X_{\mathcal{H}}$ and a fibration $\pi: M \backslash L \rightarrow S^{1}$ so that for any $\theta \in S^{1}, \pi^{-1}(\theta)$ is an embedded surface bounded by $L$, for which we can write

$$
\pi^{-1}(\theta)=u\left(S^{2} \backslash \Gamma\right)
$$

for some punctured $\widetilde{J}$-holomorphic sphere $\left[S^{2}, i, \Gamma,(a, u)\right] \in \mathcal{M}(M, \mathcal{H}, J)$ with Fredholm index $\operatorname{ind}(\tilde{u})=2$ having only positive punctures asymptotic to simply covered orbits.

Theorem 5.21 (Theorem 2.7) Assume that $(M, \mathcal{H}, J)$ admits a stable, holomorphic open book decomposition. Let $[\Sigma, j, \Gamma, \tilde{u}=(a, u)] \in \mathcal{M}(M, \mathcal{H}, J)$ be a connected pseudoholomorphic curve and assume that the image of $u$ is not a page of the open book decomposition and that $\tilde{u}$ does not have image contained in an orbit cylinder. Then at least one of the following is true:

(1) At least one of the positive punctures of $\tilde{u}$ limits to an orbit that is not a binding of the open book decomposition.

(2) At least one of the positive punctures of $\tilde{u}$ limits to a multiple cover of a binding orbit of the open book.

Proof Let $\tilde{v}=(b, v): S^{2} \backslash \Gamma^{\prime} \rightarrow \mathbb{R} \times M$ be any simple pseudoholomorphic curve which projects to a page $v\left(S^{2} \backslash \Gamma^{\prime}\right)$ of the open book decomposition. We apply (5-5) with the roles of $\tilde{u}$ and $\tilde{v}$ reversed to find that

$[\tilde{u}] *[\tilde{v}]=\sum_{z \in \Gamma_{+}} m_{z}\left(\operatorname{int}\left(\gamma_{z}, v\right)+\sum_{\substack{w \in \Gamma^{\prime} \\ \gamma_{w}=\gamma_{z}}}\left[\max \left\{\frac{\alpha\left(\gamma_{z}^{m_{z}}\right)}{m_{z}}, \alpha\left(\gamma_{w}\right)\right\}-\alpha\left(\gamma_{w}\right)\right]+d_{0}(\tilde{v} ; w)\right)$

where we have used the assumption that $\tilde{v}$ only has positive, simply-covered punctures. Moreover, by the assumption that $\operatorname{ind}(\tilde{v})=2$, we have from (5-1) that

$$
0 \leq \operatorname{wind}_{\pi}(v)+d_{0}(\tilde{v}) \leq \operatorname{ind}(\tilde{v})-\chi\left(S^{2}\right)+\# \Gamma_{\text {even }}(\tilde{v})=2-2+0=0
$$

so we conclude that $d_{0}(\tilde{v})=0$ (and that $\operatorname{wind}_{\pi}(v)=0$, but this already follows from Theorem 5.20 since $v$ parametrizes a page of the open book decomposition and is thus 
an embedding). Consequently, our formula for the generalized intersection number of $\tilde{u}$ and $\tilde{v}$ simplifies to

$$
[\tilde{u}] *[\tilde{v}]=\sum_{z \in \Gamma_{+}} m_{z}\left(\operatorname{int}\left(\gamma_{z}, v\right)+\sum_{\substack{w \in \Gamma^{\prime} \\ \gamma_{w}=\gamma_{z}}}\left[\max \left\{\frac{\alpha\left(\gamma_{z}^{m_{z}}\right)}{m_{z}}, \alpha\left(\gamma_{w}\right)\right\}-\alpha\left(\gamma_{w}\right)\right]\right)
$$

Now, the assumption that $u$ does not have image lying in a page of the open book decomposition, implies that $u$ intersects some page of the open book decomposition, which in turn, by Theorem 4.4, implies that $\tilde{u}$ has positive generalized intersection number with a pseudoholomorphic curve which projects to that page of the open book decomposition. Moreover, by homotopy invariance of the generalized intersection number, $\tilde{u}$ has positive generalized intersection number with every page of the open book decomposition. Using this observation with the formula (5-15) for the generalized intersection number of $\tilde{u}$ with a page of the open book, we see that $[\tilde{u}] *[\tilde{v}]>0$ implies that either:

(1) there exists a $z \in \Gamma$ so that $\operatorname{int}\left(\gamma_{z}, v\right)>0$ or

(2) there exists a $z \in \Gamma$ and $w \in \Gamma^{\prime}$ so that $\gamma_{z}=\gamma_{w}$ and

$$
\max \left\{\frac{\alpha\left(\gamma_{z}^{m_{z}}\right)}{m_{z}}, \alpha\left(\gamma_{w}\right)\right\}-\alpha\left(\gamma_{w}\right)>0,
$$

which in turn implies that $m_{z}>1$.

These two conditions are equivalent to the two listed in the statement of the theorem, so this completes the proof.

Remark 5.22 We remark that in the event that the first alternative of the preceding theorem does not hold, ie when all the positive punctures of the curve $\tilde{u}$ limit to coverings of binding orbits of the open book decomposition, the proof can be refined to give a bound on the covering numbers of the punctures guaranteed by the second alternative in terms of the iteration formula for the Conley-Zehnder index of the orbit in question. Indeed, given an elliptic periodic orbit $\gamma \in \mathcal{P}(M, \mathcal{H})$ and a trivialization $\Phi$ of $\left.\xi^{\mathcal{H}}\right|_{\gamma}$, Lemma 3.3 guarantees that there is an irrational $\theta$ so that

$$
\alpha^{\Phi}\left(\gamma^{k}\right)=\lfloor k \theta\rfloor \text {. }
$$

While the number $\theta$ here depends on the choice of trivialization, the change of trivialization formula from Lemma 3.4 allows us to conclude that the fractional part

$$
\{\theta\}:=\theta-\lfloor\theta\rfloor \in(0,1)
$$


of $\theta$ is independent of the choice of trivialization. We will refer to the number $\{\theta\}$ as the rotation index of $\gamma$ and denote it by $\operatorname{rot}(\gamma)$.

Now, assume that $\left[S^{2}, i, \Gamma^{\prime}, \tilde{v}=(b, v)\right]$ is a page of the open book decomposition and $[\Sigma, j, \Gamma, \tilde{u}]$ is a pseudoholomorphic curve with every positive puncture limiting to a covering of a binding orbit of the open book decomposition. Then the terms of the form $\operatorname{int}\left(\gamma_{z}, v\right)$ in formula (5-15) all vanish. Writing the remaining terms of the formula using rotation indices we find that

$$
[\tilde{u}] *[\tilde{v}]=\sum_{\substack{(z, w) \in \Gamma_{+}+\Gamma^{\prime} \\ \gamma_{z}=\gamma_{w}}}\left\lfloor m_{z} \operatorname{rot}\left(\gamma_{z}\right)\right\rfloor
$$

which the reasoning of the proof allows us to conclude must be positive. We can thus conclude that for at least one $z \in \Gamma, m_{z}>1 / \operatorname{rot}\left(\gamma_{z}\right)$.

\subsection{Generalized holomorphic curves}

In this section we prove the that the generalized intersection number of two generalized pseudoholomorphic curves with no common components in nonnegative.

Recall from the introduction that a generalized pseudoholomorphic map in $\mathbb{R} \times M$ is a quintuple $(\Sigma, j, \Gamma,(a, u), v)$ satisfying

$$
\begin{gathered}
\pi_{\xi_{\mathcal{H}}} \circ d u \circ j=J \circ \pi_{\xi_{\mathcal{H}}} \circ d u \\
u^{*} \lambda \circ j=d a+v \\
d v=d(v \circ j)=0,
\end{gathered}
$$

and a generalized pseudoholomorphic curve $[\Sigma, j, \Gamma,(a, u), v]$ is an equivalence class of generalized pseudoholomorphic maps, where two maps are considered equivalent if they differ by holomorphic reparametrization of the domain.

A key observation from [7] is that if $[\Sigma, j, \Gamma,(a, u), v]$ is a generalized pseudoholomorphic curve, then on any simply connected subset $U$ of $\Sigma$, the $M$-part $u$ of the map has a pseudoholomorphic lift. Indeed, if $h: U \rightarrow \mathbb{R}$ satisfies $d h=\left.v\right|_{U}$ then $(a+h, u): U \rightarrow \mathbb{R} \times M$ is $\widetilde{J}$-holomorphic. A consequence of this is the following, again first observed in [7], which states that for generalized pseudoholomorphic curves, one still obtains the first term of the asymptotic formula from Theorem 3.7.

Lemma 5.23 Let $(\Sigma, j, \Gamma, \tilde{u}=(a, u), v)$ be a generalized pseudoholomorphic map in $\mathbb{R} \times M$ with no component having image contained in an orbit cylinder and assume at $z \in \Gamma, u$ is asymptotic to $\gamma^{m}$. Then there is an embedding

$$
\psi:[R, \infty) \times S^{1} \rightarrow \Sigma \backslash\{z\}
$$


satisfying $\lim _{s \rightarrow \infty} \psi(s, t)=z$ so that

$$
\tilde{u}(\psi(s, t))=\left(m \tau s, \exp _{\gamma^{m}(t)} e^{\lambda s}[e(t)+r(s, t)]\right)
$$

where $\lambda<0$ is an eigenvalue of $\mathbf{A}_{\gamma^{m}, J}, e$ is an eigenvector of $\mathbf{A}_{\gamma^{m}, J}$ with eigenvalue $\lambda$ and $r(s, t) \rightarrow 0$ exponentially as $s \rightarrow \infty$.

As a consequence of this Lemma, we observe that the quantities wind ${ }_{\infty}^{\Phi}$ and $d_{0}=$ wind $_{\infty}^{\Phi}-\alpha^{\Phi}$, defined in (3-16) and (3-18) respectively are well-defined for ends of generalized pseudoholomorphic curves, provided the images of the maps are not orbit cylinders.

We can now prove Theorem 2.9 which we restate here.

Theorem 5.24 (Global positivity of intersections for generalized holomorphic curves) Let $[\Sigma, j, \Gamma, \tilde{u}=(a, u), v],\left[\Sigma^{\prime}, j^{\prime}, \Gamma^{\prime}, \tilde{v}=(b, v), v^{\prime}\right] \in \mathcal{M}_{\Delta}(M, \mathcal{H}, J)$ be generalized pseudoholomorphic curves and assume that no component of $\tilde{u}$ or $\tilde{v}$ is contained in an orbit cylinder. Then

$$
[\tilde{u}] *[\tilde{v}] \geq 0
$$

Proof As a consequence of Lemma 5.23, we know that $\tilde{u}$ and $\tilde{v}$ have ends that wind, so Theorem 5.8 tells us that the generalized intersection number of $\tilde{u}$ and $\tilde{v}$ is given by

$$
\begin{aligned}
{[\tilde{u}] *[\tilde{v}]=} & \sum_{w \in \Gamma_{+}^{\prime}}\left|m_{w}\right|\left(\operatorname{int}\left(\gamma_{w}, u\right)+\sum_{\substack{z \in \Gamma_{+} \\
\gamma_{z}=\gamma_{w}}}\left|m_{z}\right| \bar{\Lambda}\left(\frac{\alpha\left(\gamma_{w}^{m_{w}}\right)}{\left|m_{w}\right|}, \frac{\alpha\left(\gamma_{z}^{m_{z}}\right)}{\left|m_{z}\right|}\right)+d_{0}(\tilde{u} ; z)\right) \\
& +\sum_{z \in \Gamma_{-}}\left|m_{z}\right|\left(\operatorname{int}\left(\gamma_{z}, v\right)+\sum_{\substack{w \in \Gamma^{\prime} \\
\gamma_{w}=\bar{\gamma}_{z}}}\left|m_{w}\right| \bar{\Lambda}\left(\frac{\alpha\left(\gamma_{z}^{m z}\right)}{\left|m_{z}\right|}, \frac{\alpha\left(\gamma_{w}^{m}\right)}{\left|m_{w}\right|}\right)+d_{0}(\tilde{v} ; w)\right) \\
& +\sum_{\substack{(z, w) \in \Gamma_{-} \times \Gamma_{+}^{\prime} \\
\gamma_{z}=\gamma_{w}}}\left|m_{w}\right| d_{0}(\tilde{u} ; z)+\left|m_{z}\right| d_{0}(\tilde{v} ; w)-\left|m_{w} m_{z}\right|\left[\frac{\alpha\left(\gamma_{z}^{m}\right)}{\left|m_{z}\right|}+\frac{\alpha\left(\gamma_{w}^{m w}\right)}{\left|m_{w}\right|}\right],
\end{aligned}
$$

with $\bar{\Lambda}$ as defined in (5-6). The theorem would follow immediately if we knew that each term appearing in this formula were nonnegative. The nonnegativity of the $d_{0}$ terms is an immediate consequence of the asymptotic description from Lemma 5.23 and reasoning identical to that in Lemma 3.13. Every other term has previously been shown to be nonnegative in the proof of Corollary 5.9 except for the terms $\operatorname{int}\left(\gamma_{w}, u\right)$ and $\operatorname{int}\left(\gamma_{z}, v\right)$ since in Corollary 5.9 we were assuming that $\tilde{u}$ and $\tilde{v}$ were 
pseudoholomorphic, while here we only assume generalized pseudoholomorphic. It remains true in this case that intersections of a projected generalized pseudoholomorphic curves with a periodic orbit of $X_{\mathcal{H}}$ always occur with positive local intersection index. Indeed, assume at $z_{*} \in \Sigma$ that $u\left(z_{*}\right)=\gamma(t)$ where $\gamma$ is a periodic orbit or $X_{\mathcal{H}}$. Then let $\bar{u}=(a+h, u): U \rightarrow \mathbb{R} \times M$ be a local pseudoholomorphic lift of $u$ on a neighborhood $U$ of $z_{*}$. Then $\bar{u}\left(z_{*}\right)$ intersects the orbit cylinder $\tilde{\gamma}$. By the assumption that $\tilde{u}$ does not have any components with image contained in an orbit cylinder, it follows that the intersection of $\bar{u}$ with $\tilde{\gamma}$ is isolated and has positive local index and it follows as in (5-4) that the intersection of $\gamma$ with $u$ is isolated and has positive local index.

\section{Appendix A Zeroes of $\pi_{\xi \mathcal{H}} \circ d u$}

Here we will prove that for a connected pseudoholomorphic curve $[\Sigma, j, \Gamma,(a, u)] \in$ $\mathcal{M}(M, \mathcal{H}, J)$, the projection of the derivative of $u$ onto the hyperplane distribution $\xi^{\mathcal{H}}$ either vanishes identically or has a finite number of isolated zeroes of finite positive order. This is proved in [8] in the case that the Hamiltonian structure comes from a contact form and the proof here is an adaptation of the argument given there.

We start with a local coordinate lemma which is a straightforward modification of the well known version of Darboux's theorem for presymplectic manifolds. Because it may be of independent interest, we prove the result for stable Hamiltonian structures on manifolds of arbitrary odd dimension. In the definition of stable Hamiltonian structure for a $2 n+1$-dimensional manifold $M$, the condition (H1) needs to be changed to

$$
\lambda \wedge \omega^{n}>0
$$

but otherwise remains the same. The definitions of $X_{\mathcal{H}}$ and $\xi^{\mathcal{H}}$ are identical and it remains true that $\lambda$ and $\omega$ are preserved by the flow of $X_{\mathcal{H}}$. For more details see eg $[2 ; 22]$. For the following we equip $\mathbb{R}^{2 n+1}$ with the coordinates $\{(z, x, y)\}=$ $\left\{\left(z, x_{i}, y_{i}\right)\right\} \in \mathbb{R} \times \mathbb{R}^{n} \times \mathbb{R}^{n}$.

Lemma A.1 (Darboux's theorem for stable Hamiltonian structures) Let $(M, \mathcal{H})$ be a closed, $2 n+1$-dimensional manifold equipped with a stable Hamiltonian structure $(\lambda, \omega)$. For any $p_{0} \in M$, there exists an $\varepsilon>0$ and an embedding

$$
\phi:(-\varepsilon, \varepsilon)^{2 n+1} \rightarrow M
$$

with $\phi(0)=p_{0}$ and

$$
\phi^{*} \lambda=d z-\sum_{i=1}^{n}\left(g_{i}(x, y) d x_{i}+h_{i}(x, y) d y_{i}\right)
$$




$$
\phi^{*} \omega=\omega_{0}=\sum_{i=1}^{n} d x_{i} \wedge d y_{i}
$$

where the $g_{i}, h_{i}:(-\varepsilon, \varepsilon)^{2 n} \rightarrow \mathbb{R}$ are smooth real-valued functions satisfying $g_{i}(0)=$ $h_{i}(0)=0$.

Proof Let $\psi: \mathbb{R} \times M \rightarrow M$ denote the flow of $X_{\mathcal{H}}$, ie $\dot{\psi}_{t}(p)=X_{\mathcal{H}} \circ \psi_{t}(p)$ for all $(t, p) \in \mathbb{R} \times M$. Let $\left\{e_{i}, f_{i}\right\}$ be a symplectic basis for $\left(\xi_{p_{0}}^{\mathcal{H}}, \omega\right)$, that is, assume that

$$
\omega\left(e_{i}, e_{j}\right)=\omega\left(f_{i}, f_{j}\right)=0
$$

for any $i, j$ and that

$$
\omega\left(e_{i}, f_{j}\right)=\delta_{i j}
$$

Define a map $\phi_{0}: \mathbb{R}^{2 n+1} \rightarrow M$ by

$$
\phi_{0}\left(z, x_{i}, y_{i}\right)=\psi_{z}\left(\exp _{p_{0}}\left(\sum_{i=1}^{n} x_{i} e_{i}+y_{i} f_{i}\right)\right)
$$

where exp is the exponential map of any Riemannian metric on $M$. It follows from the definition that $\phi_{0}$ satisfies:

- $d \phi_{0}(z, x, y) \partial_{z}=X_{\mathcal{H}}$ for all $(z, x, y) \in \mathbb{R}^{2 n+1}$,

- $d \phi_{0}(0) \partial_{x_{i}}=e_{i}$ and $d \phi_{0}(0) \partial_{y_{i}}=f_{i}$ and thus

- $\phi_{0}$ is an embedding on some neighborhood of the origin,

- $\phi_{0}^{*}(0) \omega=\omega_{0}$ and $\phi_{0}^{*}(0) \lambda=d z$.

Since the flow of $X_{\mathcal{H}}$ preserves $\lambda$ and $\omega$, we can conclude that $\phi_{0}^{*} \lambda$ and $\phi_{0}^{*} \omega$ are independent of the $z$-variable. Since $\phi_{0}^{*} \lambda\left(\partial_{z}\right)=\lambda\left(X_{\mathcal{H}}\right)=1$, we thus have

$$
\begin{gathered}
\phi_{0}^{*} \lambda=d z-\sum_{i=1}^{n}\left(\tilde{g}_{i}(x, y) d x_{i}+\tilde{h}_{i}(x, y) d y_{i}\right) \\
\phi_{0}^{*} \omega=\omega_{0}+r_{(x, y)}
\end{gathered}
$$

where $\tilde{g}_{i}, \tilde{h}_{i}$ are smooth, real-values functions on some neighborhood of the origin in $\mathbb{R}^{2 n}$ satisfying $\tilde{g}_{i}(0)=\tilde{h}_{i}(0)=0$ and where $r_{(x, y)}$ is a two-form on $\mathbb{R}^{2 n}$ satisfying $r_{(0,0)}=0$. The result then follows from applying a Moser trick in the $x$ and $y$ variables (see eg [13, Theorem 1, Section 1.3]). 
Next we show that for $[\Sigma, j, \Gamma,(a, u)] \in \mathcal{M}(M, \mathcal{H}, J), \pi_{\xi \mathcal{H}} \circ d u$ can only have isolated zeroes of positive order. It suffices to prove this in "Darboux coordinates" provided by the preceding lemma. In the following $\mathbb{D}_{\varepsilon}$ will denote the disk of radius $\varepsilon$ in $\mathbb{C}=\{s+i t\}$. We observe this argument readily generalizes to higher dimensions, but is of most use in dimension 3 in which case the algebraic count of zeroes of $\pi_{\xi_{\mathcal{H}}} \circ d u$ is topologically controlled.

Lemma A.2 Let $g, h: \mathbb{R}^{2} \rightarrow \mathbb{R}$ be smooth functions and let $J \in \mathcal{J}\left(\mathbb{R}^{3}, \mathcal{H}_{0}\right)$ where $\mathcal{H}_{0}=\left(\lambda_{0}, \omega_{0}\right)$ is the Hamiltonian structure defined by

$$
\begin{gathered}
\lambda_{0}=d z-g(x, y) d x-h(x, y) d y \\
\omega_{0}=d x \wedge d y .
\end{gathered}
$$

Moreover, let $\pi: \mathbb{R} X_{\mathcal{H}_{0}} \oplus \xi^{\mathcal{H}_{0}} \rightarrow \xi^{\mathcal{H}_{0}}$ be the projection onto $\xi^{\mathcal{H}_{0}}=\operatorname{ker} \lambda_{0}$ along $X_{\mathcal{H}_{0}}$. If $\tilde{u}=(a, u):\left(\mathbb{D}_{\varepsilon}, i\right) \rightarrow\left(\mathbb{R} \times \mathbb{R}^{3}, \widetilde{J}\right)$ is a pseudoholomorphic map, then $\pi \circ d u$ either vanishes identically or has isolated zeroes of finite positive order.

Proof We will show that in an appropriate basis for $\xi^{\mathcal{H}_{0}}$ the section $(\pi \circ d u)\left(\partial_{s}\right)$ satisfies a perturbed Cauchy-Riemann equation. The result will then follow from the similarity principle (see eg [13, Appendix A.6]).

We first note that the vector fields $e=\partial_{x}+g \partial_{z}$ and $f=\partial_{y}+h \partial_{z}$ form a basis for $\xi^{\mathcal{H}_{0}}$ and that $X_{\mathcal{H}_{0}}=\partial_{z}$. Moreover, for any vector field $v=v^{x} \partial_{x}+v^{y} \partial_{y}+v^{z} \partial_{z}$ on $\mathbb{R}^{3}$ we have that

$$
\begin{aligned}
\pi v & =v-\lambda_{0}(v) X_{\mathcal{H}_{0}} \\
& =v^{x} \partial_{x}+v^{y} \partial_{y}+\left(v^{x} g+v^{y} h\right) \partial_{z} \\
& =v^{x} e+v^{y} f
\end{aligned}
$$

so the coordinates of $\pi v$ in the basis $\{e, f\}$ are given by the $x$ and $y$ components of $v$ in the standard basis for $\mathbb{R}^{3}$.

Now, writing $u=\left(u^{z}, u^{x}, u^{y}\right) \in \mathbb{R}^{3}$ and $v=\left(u^{x}, u^{y}\right)$, the equation $\pi \circ d u \circ i=$ $J \circ \pi \circ d u$ applied to $\partial_{s}$ and expressed in the basis $\{e, f\}$ becomes

$$
v_{s}+\bar{J}(s, t) v_{t}=0
$$

Here $\bar{J}(s, t)$ is $J(u(s, t))$ represented in the basis $\{e, f\}$ and hence satisfies $\bar{J}^{2}=-I$. Letting $w=v_{s}$ and differentiating the above equation with respect to $s$ leads to

$$
w_{s}+\bar{J}(s, t) w_{t}+A(s, t) w=0
$$


with

$$
A(s, t)=\bar{J}_{s}(s, t) \bar{J}(s, t) .
$$

As explained in the first paragraph, the result is now an easy consequence of the similarity principle.

Corollary A.3 Let $[\Sigma, j, \Gamma, \tilde{u}=(a, u)] \in \mathcal{M}(M, \mathcal{H}, J)$ be a connected pseudoholomorphic curve for some cylindrical $\widetilde{J}$ associated to a $J \in \mathcal{J}(M, \mathcal{H})$ and let $\pi_{\xi^{\mathcal{H}}}: T M=\mathbb{R} X_{\mathcal{H}} \oplus \xi^{\mathcal{H}} \rightarrow \xi^{\mathcal{H}}$ be the projection onto $\xi^{\mathcal{H}}$ along $X_{\mathcal{H}}$. Then $\pi_{\xi^{\mathcal{H}}} \circ d u$ either vanishes identically or has a finite number of isolated zeroes each of finite positive order.

Proof Assume that $\pi_{\xi_{\mathcal{H}}} \circ d u$ does not vanish identically. By the previous two lemmas, the zeroes of $\pi_{\xi \mathcal{H}} \circ d u$ must be isolated and of finite positive order. Moreover, according to Corollary $3.12, \pi_{\xi_{\mathcal{H}}} \circ d u$ is nonvanishing in some neighborhood of each puncture since we assume it does not vanish identically on $\Sigma \backslash \Gamma$. We conclude that $\pi_{\xi \mathcal{H}} \circ d u$ has a finite number of isolates zeroes of finite positive order.

\section{Appendix B Local intersections of generalized pseudoholo- morphic curves}

In this appendix, we construct local examples of generalized pseudoholomorphic curves exhibiting intersection behavior that cannot occur for (genuine) pseudoholomorphic curves. The author first learned that such examples should exist from C. Abbas.

We consider $\mathbb{R}^{3}=\{(z, x, y)\}$ equipped with the stable Hamiltonian structure $\mathcal{H}=$ $(\lambda, \omega)=(d z, d x \wedge d y)$. Then $X_{\mathcal{H}}=\partial_{z}$ and $\xi^{\mathcal{H}}=\operatorname{span}\left\{\partial_{x}, \partial_{y}\right\}$. Define $J$ on $\xi^{\mathcal{H}}$ by $J \partial_{x}=\partial_{y}$. The projection $\pi_{\xi_{\mathcal{H}}}: T \mathbb{R}^{3}=\mathbb{R} X_{\mathcal{H}} \oplus \xi^{\mathcal{H}} \rightarrow \xi^{\mathcal{H}}$ is given by

$$
(z, x, y) \mapsto(x, y) .
$$

Letting $\mathbb{D}$ denote the unit disk in $\mathbb{C}=\{s+i t\}$, the generalized holomorphic curve equations (2-8) for a map $\tilde{u}=\left(a, u_{1}, u_{2}\right): \mathbb{D} \rightarrow \mathbb{R} \times \mathbb{R} \times \mathbb{R}^{2}$ reduce to

$$
\left\{\begin{array}{c}
d\left(d u_{1} \circ i-d a\right)=0 \\
d\left(d u_{1}+d a \circ i\right)=0 \\
\partial_{s} u_{2}+J \partial_{t} u_{2}=0
\end{array}\right.
$$

Consider maps $\tilde{u}, \tilde{v}: \mathbb{D} \rightarrow \mathbb{R} \times \mathbb{R}^{3}$ defined by

$$
\tilde{u}(s, t)=(0,0, s, t)
$$


and

$$
\tilde{v}(s, t)=(c s, t, s, t)
$$

where $c \in \mathbb{R}$ is constant. Then it is straightforward to check that $\tilde{u}$ and $\tilde{v}$ satisfy (B-1) and we moreover observe that $\tilde{u}$ is genuinely holomorphic. If $c=0$ then $\tilde{u}$ and $\tilde{v}$ intersect along the line $\tilde{u}(s, 0)=\tilde{v}(s, 0)=(0,0, s, 0)$, which cannot happen for two genuinely pseudoholomorphic maps. If $c=-1$, then $\tilde{u}$ and $\tilde{v}$ have an isolated transverse intersection at $\tilde{u}(0,0)=\tilde{v}(0,0)=(0,0,0,0)$ and the sign of the intersection is given by

$$
\operatorname{det}\left[\begin{array}{lllll}
\partial_{s} \tilde{u} & \partial_{t} \tilde{u} & \partial_{s} \tilde{v} & \partial_{t} \tilde{v}
\end{array}\right](0,0)=\operatorname{det}\left[\begin{array}{cccc}
0 & 0 & -1 & 0 \\
0 & 0 & 0 & 1 \\
1 & 0 & 1 & 0 \\
0 & 1 & 0 & 1
\end{array}\right]=-1
$$

so the local intersection number is -1 .

\section{References}

[1] A Abbondandolo, Morse theory for Hamiltonian systems, Research Notes in Mathematics 425, Chapman \& Hall, Boca Raton, FL (2001) MR1824111

[2] F Bourgeois, Y Eliashberg, H Hofer, K Wysocki, E Zehnder, Compactness results in symplectic field theory, Geom. Topol. 7 (2003) 799-888 MR2026549

[3] D L Dragnev, Fredholm theory and transversality for noncompact pseudoholomorphic maps in symplectizations, Comm. Pure Appl. Math. 57 (2004) 726-763 MR2038115

[4] Y Eliashberg, A Givental, H Hofer, Introduction to symplectic field theory, Geom. Funct. Anal. (2000) 560-673 MR1826267 GAFA 2000 (Tel Aviv, 1999)

[5] Y Eliashberg, S S Kim, L Polterovich, Geometry of contact transformations and domains: orderability versus squeezing, Geom. Topol. 10 (2006) 1635-1747 MR2284048

[6] M Gromov, Pseudoholomorphic curves in symplectic manifolds, Invent. Math. 82 (1985) 307-347 MR809718

[7] H Hofer, Holomorphic curves and real three-dimensional dynamics, Geom. Funct. Anal. (2000) 674-704 MR1826268 GAFA 2000 (Tel Aviv, 1999)

[8] H Hofer, K Wysocki, E Zehnder, Properties of pseudo-holomorphic curves in symplectisations. II. Embedding controls and algebraic invariants, Geom. Funct. Anal. 5 (1995) 270-328 MR1334869

[9] H Hofer, K Wysocki, E Zehnder, Properties of pseudoholomorphic curves in symplectisation. IV. Asymptotics with degeneracies, from: "Contact and symplectic geometry (Cambridge, 1994)”, Publ. Newton Inst. 8, Cambridge Univ. Press, Cambridge (1996) 78-117 MR1432460 
[10] H Hofer, K Wysocki, E Zehnder, Properties of pseudoholomorphic curves in symplectisations. I. Asymptotics, Ann. Inst. H. Poincaré Anal. Non Linéaire 13 (1996) 337-379 MR1395676

[11] H Hofer, K Wysocki, E Zehnder, Properties of pseudoholomorphic curves in symplectizations. III. Fredholm theory, from: "Topics in nonlinear analysis", Progr. Nonlinear Differential Equations Appl. 35, Birkhäuser, Basel (1999) 381-475 MR1725579

[12] H Hofer, K Wysocki, E Zehnder, Finite energy foliations of tight three-spheres and Hamiltonian dynamics, Ann. of Math. (2) 157 (2003) 125-255 MR1954266

[13] H Hofer, E Zehnder, Symplectic invariants and Hamiltonian dynamics, Birkhäuser Advanced Texts: Basler Lehrbücher. [Birkhäuser Advanced Texts: Basel Textbooks], Birkhäuser Verlag, Basel (1994) MR1306732

[14] M Hutchings, An index inequality for embedded pseudoholomorphic curves in symplectizations, J. Eur. Math. Soc. (JEMS) 4 (2002) 313-361 MR1941088

[15] M Hutchings, The embedded contact homology index revisited, from: "New perspectives and challenges in symplectic field theory", CRM Proc. Lecture Notes 49, Amer. Math. Soc. (2009) 263-297 MR2555941

[16] M Kriener, An intersection formula for finite energy half cylinders, PhD thesis, ETH Zurich (1998)

[17] D McDuff, Singularities and positivity of intersections of J-holomorphic curves, from: "Holomorphic curves in symplectic geometry", Progr. Math. 117, Birkhäuser, Basel (1994) 191-215 MR1274930 With an appendix by Gang Liu

[18] MJ Micallef, B White, The structure of branch points in minimal surfaces and in pseudoholomorphic curves, Ann. of Math. (2) 141 (1995) 35-85 MR1314031

[19] A Momin, Contact homology of orbit complements and implied existence arXiv: 1012.1386

[20] E Mora-Donato, Pseudoholomorphic cylinders in symplectisations, PhD thesis, New York University (2003) MR2704613

[21] R L Siefring, Intersection theory of finite energy surfaces, PhD thesis, New York University (2005) MR2708114

[22] R Siefring, Relative asymptotic behavior of pseudoholomorphic half-cylinders, Comm. Pure Appl. Math. 61 (2008) 1631-1684 MR2456182

Max Planck Institute for Math in Sci, Inselstraße 22, D-04103 Leipzig, Germany Department of Mathematics, Michigan State University, East Lansing, MI 48824, USA siefring@mis.mpg.de http://personal-homepages.mis.mpg.de/siefring/

Proposed: Yasha Eliashberg Received: 4 June 2010 Seconded: Ronald Fintushel, Leonid Polterovich Revised: 19 June 2011 